\title{
Divergent Synthesis of Dihydroxanthene-Hemicyanine Fused Near-Infrared Fluorophores through the Late-Stage Amination of a Bifunctional Precursor
}

Michelle Jui Hsien Ong, ${ }^{\dagger}$ Rajavel Srinivasan $\dagger,{ }^{*}, \dagger$ Anthony Romieu, ${ }^{*},, \S$ and JeanAlexandre Richard ${ }^{*}+$

${ }^{\dagger}$ Organic Chemistry, Institute of Chemical and Engineering Sciences (ICES), Agency for Science, Technology and Research (A*STAR), 8 Biomedical Grove, Neuros, \#07-01, 138665, Singapore

${ }^{\ddagger}$ ICMUB, UMR 6302, CNRS, Univ. Bourgogne Franche-Comté, 9, Avenue Alain Savary, 21078 Dijon cedex, France

${ }^{\S}$ Institut Universitaire de France, 103 Boulevard Saint-Michel, 75005 Paris, France

†Current address: School of Pharmaceutical Science and Technology Tianjin University, Building 24, 92 Weijin Road, Nankai District Tianjin, 300072 P.R. China

\section{Supporting Information}


General

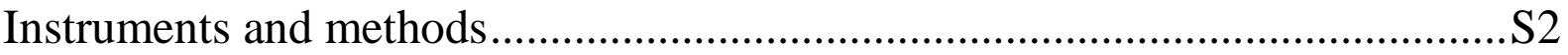

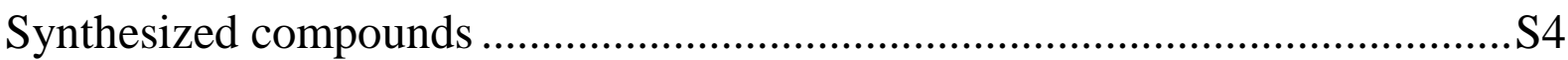

${ }^{1} \mathrm{H}$ and ${ }^{13} \mathrm{C}$ NMR spectra of synthesized compounds......................................... 19

Absorption, excitation and emission spectra of DHX-hemicyanine fused NIR

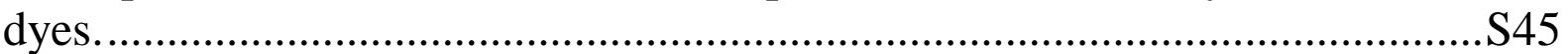

\section{General}

All reactions were carried out under a nitrogen or argon atmosphere with dry solvents under anhydrous conditions, unless otherwise noted. Methanol $(\mathrm{MeOH}), 1,4$-dioxane and $\mathrm{N}, \mathrm{N}$ dimethylformamide (DMF) were purchased in anhydrous form and used without further purification. Water, ethyl acetate (EtOAc), diethyl ether $\left(\mathrm{Et}_{2} \mathrm{O}\right)$, methylene chloride $\left(\mathrm{CH}_{2} \mathrm{Cl}_{2}\right)$, and hexanes were purchased at the highest commercial quality and used without further purification. Reagents were purchased at the highest commercial quality and used without further purification. NO2AtBu (di-tert-butyl 2,2'-(1,4,7-triazacyclononane-1,4diyl)diacetate) was synthesized and provided by the CheMatech company (Dijon, France, http://www.chematech-mdt.com/). Yields refer to chromatographically and spectroscopically ( ${ }^{1} \mathrm{H}$ NMR) homogeneous materials. Reactions were monitored by thin-layer chromatography (TLC) carried out on $0.25 \mathrm{~mm}$ E. Merck silica gel plates (60F-254) using UV light as visualizing agent and a solution of potassium permanganate and heat as developing agents. E. Merck silica gel (60, particle size $0.040-0.063 \mathrm{~mm})$ was used for flashcolumn chromatography. Dimethylsulfoxide (DMSO, $+99.9 \%$, for spectroscopy), ethanol (EtOH, absolute anhydrous RPE) and chloroform $\left(\mathrm{CHCl}_{3}, \mathrm{RPE}\right.$ for analysis ISO, stabilized with EtOH 0.6-1\%) were purchased from Acros and Carlo Erba respectively. Bovine serum albumin (BSA, heat shock fraction, $\mathrm{pH} 7, \geq 98 \%$ ) was provided by Sigma-Aldrich. Phosphate buffered saline (PBS, $100 \mathrm{mM}$ phosphate $+150 \mathrm{mM} \mathrm{NaCl}$, $\mathrm{pH}$ 7.5) was prepared using water purified with a PURELAB Ultra system from ELGA (purified to $18.2 \mathrm{M} \Omega . \mathrm{cm}$ ).

\section{Instruments and methods}

NMR spectra were recorded on a Bruker DRX-400 (400 MHz) instrument and calibrated using residual non-deuterated solvent as an internal reference. ${ }^{1}$ The following abbreviations 
were used to explain the multiplicities: $\mathrm{s}=$ singlet, $\mathrm{d}=$ doublet, $\mathrm{t}=$ triplet, $\mathrm{q}=$ quartet, quint $=$ quintet, $\mathrm{m}=$ multiplet, pent $=$ pentet, hex $=$ hexet, $\mathrm{br}=$ broad. IR spectra were recorded on $\mathrm{a}$ Perkin-Elmer Spectrum One FTIR spectrometer with diamond ATR accessory. Bond vibration frequencies are expressed in reciprocal centimeters $\left(\mathrm{cm}^{-1}\right)$. High-resolution mass spectra (HRMS) were recorded on an Agilent ESI TOF (time-of-flight) mass spectrometer at $3.5 \mathrm{kV}$ emitter voltage. UV-visible spectra were obtained either on a Varian Cary 50 scan (single-beam) or Varian Cary 5000 UV-vis-NIR (dual-beam) spectrophotometer by using a rectangular quartz cell (Hellma, 100-QS, $45 \times 12.5 \times 12.5 \mathrm{~mm}$, pathlength $10 \mathrm{~mm}$, chamber volume: $3.5 \mathrm{~mL}$ ), at $25{ }^{\circ} \mathrm{C}$ (using a temperature control system combined with water circulation). Fluorescence spectra (emission/excitation spectra) were recorded with an HORIBA Jobin Yvon Fluorolog spectrophotometer (software FluorEssence) at $25^{\circ} \mathrm{C}$ (using a temperature control system combined with water circulation), using a standard fluorometer cell (Labbox, LB Q, $10 \mathrm{~mm}$ ). Emission spectra were recorded in the range 670-900 nm after excitation at $650 \mathrm{~nm}$ (shutter: Auto Open, excitation slit width $=5 \mathrm{~nm}$ and emission slit width $=5 \mathrm{~nm}$ ). Excitation spectra were recorded in the range $400-810 \mathrm{~nm}$ after emission at $830 \mathrm{~nm}$ (shutter: Auto Open, excitation slit width $=5 \mathrm{~nm}$ and emission slit width $=12 \mathrm{~nm}$ ). All fluorescence spectra were corrected until $850 \mathrm{~nm}$. Fluorescence quantum yields were measured at $25{ }^{\circ} \mathrm{C}$ by a relative method using indocyanine green (ICG, $\Phi_{\mathrm{F}}=10.6 \%$ in DMSO) as a standard (dilution by a factor of 3 between absorption and fluorescence measurements). The following equation was used to determine the relative fluorescence quantum yield:

$$
\Phi_{\mathrm{F}}(\mathrm{x})=\left(f_{\mathrm{S}} / f_{\mathrm{X}}\right)\left(\mathrm{F}_{\mathrm{X}} / \mathrm{F}_{\mathrm{S}}\right)\left(\mathrm{n}_{\mathrm{X}} / \mathrm{n}_{\mathrm{S}}\right)^{2} \Phi_{\mathrm{F}}(\mathrm{s})
$$

where $f$ is the absorption factor $\left(f_{x}=1-10^{-\mathrm{Ax}}\right.$, where $\mathrm{A}=$ absorbance $)$, A is the absorbance (in the range of 0.01-0.1 A.U.), $\mathrm{F}$ is the area under the emission curve, $\mathrm{n}$ is the refractive index of the solvents (at $25{ }^{\circ} \mathrm{C}$ ) used in measurements, and the subscripts $\mathrm{s}$ and $\mathrm{x}$ represent standard and unknown, respectively. The following refractive index values were used: 1.479 for DMSO, 1.337 for PBS + 5\% BSA, 1.361 for $\mathrm{EtOH}$ and 1.446 for $\mathrm{CHCl}_{3}$. Stock solutions $(1.0 \mathrm{mg} / \mathrm{mL})$ of dihydroxanthene-hemicyanine fused NIR dyes were prepared in DMSO and subsequently diluted with $\mathrm{PBS}+5 \% \mathrm{BSA}$, EtOH or $\mathrm{CHCl}_{3}$ for $\mathrm{UV}$-vis absorption and fluorescence measurements. 


\section{Synthesized compounds}

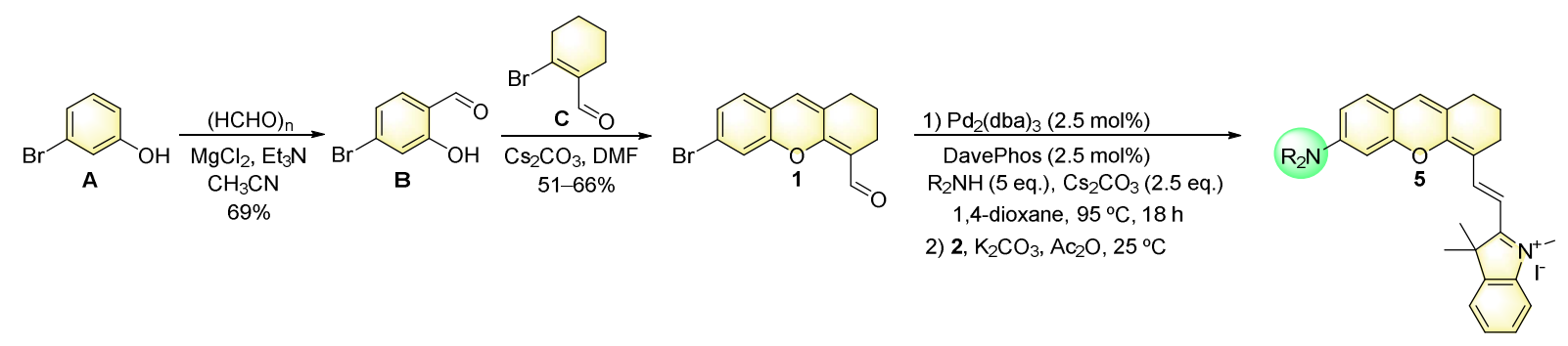

Scheme S1. Synthetic route towards DHX NIR fluorophores 5.

4-bromo-2-hydroxybenzaldehyde (B). To 3-bromophenol (A) (1 g, $5.78 \mathrm{mmol}$ ) in dry $\mathrm{CH}_{3} \mathrm{CN}(30 \mathrm{~mL})$ at $25^{\circ} \mathrm{C}$ were added $\mathrm{MgCl}_{2}(0.85 \mathrm{~g}, 8.92 \mathrm{mmol})$ and triethylamine $(3.2 \mathrm{~mL}$, $22.9 \mathrm{mmol})$ and the solution was stirred for $20 \mathrm{~min}$. Paraformaldehyde (1.25 g, $41.6 \mathrm{mmol})$ was added and the mixture was refluxed at $100{ }^{\circ} \mathrm{C}$ for $18 \mathrm{~h}$. Water was added and the mixture was acidified with diluted hydrochloric acid to $\mathrm{pH}$ 2. The solution was extracted with $\mathrm{Et}_{2} \mathrm{O}$ $(50 \mathrm{~mL} \times 2)$ and the combined organic layers were washed with saturated $\mathrm{NaCl}(50 \mathrm{~mL} \times 2)$ and dried over anhydrous $\mathrm{Na}_{2} \mathrm{SO}_{4}$, filtered and concentrated in vacuo. Purification on silica gel (10\% EtOAc in hexane, $\mathrm{R}_{\mathrm{f}}$ 0.65) afforded 4-bromo-2-hydroxybenzaldehyde $(\mathbf{B})$ as a white solid (0.8 g, 69\%). ${ }^{1} \mathrm{H}$ NMR $\left(400 \mathrm{MHz}, \mathrm{CDCl}_{3}\right) \delta=11.11(\mathrm{~s}, 1 \mathrm{H}), 9.86(\mathrm{~s}, 1 \mathrm{H}), 7.41(\mathrm{~d}, J=$ $8.2 \mathrm{~Hz}, 1 \mathrm{H}), 7.22-7.13(\mathrm{~m}, 2 \mathrm{H}) .{ }^{13} \mathrm{C} \mathrm{NMR}\left(100 \mathrm{MHz}, \mathrm{CDCl}_{3}\right) \delta=195.9,162.2,134.6,132.2$, $123.7,121.3,119.7 \mathrm{ppm}$. The spectral data matched that previously obtained. ${ }^{3}$

6-Bromocyclohex-1-ene-1-carbaldehyde (C). To DMF (12 mL, $155 \mathrm{mmol})$ and $\mathrm{CHCl}_{3}(50$ $\mathrm{mL})$ at $0{ }^{\circ} \mathrm{C}$ was added $\mathrm{PBr}_{3}(9 \mathrm{~mL}, 94.7 \mathrm{mmol})$ slowly. After $1 \mathrm{~h}$, cyclohexanone $(4 \mathrm{~mL}, 39$ mmol) in solution in $\mathrm{CHCl}_{3}(10 \mathrm{~mL})$ was added and the mixture was stirred at $25^{\circ} \mathrm{C}$ for $18 \mathrm{~h}$. The resulting red solution was then poured onto ice and solid $\mathrm{NaHCO}_{3}$ was slowly added until $\mathrm{pH} \sim 7$. The layers were separated and the aqueous layer was extracted with $\mathrm{CH}_{2} \mathrm{Cl}_{2}(100$ $\mathrm{mL}$ ). The organic layer was dried over anhydrous $\mathrm{Na}_{2} \mathrm{SO}_{4}$, filtered and concentrated in vacuo. The chemical purity of the crude yellow oil was good and could be used directly in the next step without further purification. ${ }^{1} \mathrm{H} \mathrm{NMR}\left(400 \mathrm{MHz}, \mathrm{CDCl}_{3}\right) \delta 10.02(\mathrm{~s}, 1 \mathrm{H}), 2.75(\mathrm{t}, J=2.3$ 
$\mathrm{Hz}, 2 \mathrm{H}), 2.31-2.24(\mathrm{~m}, 2 \mathrm{H}), 1.80-1.65(\mathrm{~m}, 4 \mathrm{H}) ;{ }^{13} \mathrm{C} \mathrm{NMR}\left(100 \mathrm{MHz}, \mathrm{CDCl}_{3}\right) \delta=193.8$, $143.6,135.5,39.0,25.2,24.4,21.3 \mathrm{ppm}$. The spectral data matched that previously obtained. ${ }^{4}$

6-bromo-2,3-dihydro-1H-xanthene-4-carbaldehyde (1). To $\mathrm{Cs}_{2} \mathrm{CO}_{3}(1.2 \mathrm{~g}, 3.68 \mathrm{mmol})$ and 4-bromo-2-hydroxybenzaldehyde (B) (0.26 g $1.26 \mathrm{mmol})$ was added DMF (10 mL). 6Bromocyclohex-1-ene-1-carbaldehyde $(\mathbf{C})(0.49 \mathrm{~g}, 2.56 \mathrm{mmol})$ in DMF (1 mL) was added slowly and the mixture was stirred at $25^{\circ} \mathrm{C}$ for $48 \mathrm{~h}$. The resulting mixture was filtered under vacuum, washed with $\mathrm{H}_{2} \mathrm{O}(20 \mathrm{~mL})$ and extracted with EtOAc $(50 \mathrm{~mL})$. The combined organic layers were dried over anhydrous $\mathrm{Na}_{2} \mathrm{SO}_{4}$, filtered and concentrated in vacuo. Purification on silica gel (20\% EtOAc in hexane, $\mathrm{R}_{\mathrm{f}}$ 0.5) afforded 6-bromo-2,3-dihydro-1Hxanthene-4-carbaldehyde (1) as a yellow solid (0.19 g, 51\%). IR (film) $v_{\max }=2940,2845$, 1646. 1620, 1568, 1389, 1235, 1176, 1125, 1066, 1052, 925, $733 \mathrm{~cm}^{-1} ;{ }^{1} \mathrm{H}$ NMR (400 MHz, $\left.\mathrm{CDCl}_{3}\right) \delta=10.32(\mathrm{~s}, 1 \mathrm{H}), 7.30-7.27(\mathrm{~m}, 1 \mathrm{H}), 7.20(\mathrm{dd}, J=8.1,1.9 \mathrm{~Hz}, 1 \mathrm{H}), 7.01(\mathrm{~d}, J=8.2$ $\mathrm{Hz}, 1 \mathrm{H}), 6.62(\mathrm{~s}, 1 \mathrm{H}), 2.57(\mathrm{t}, J=6.6,1.5 \mathrm{~Hz}, 2 \mathrm{H}), 2.44(\mathrm{t}, J=6.1 \mathrm{~Hz}, 2 \mathrm{H}), 1.73(\mathrm{p}, J=6.2$ $\mathrm{Hz}, 2 \mathrm{H}) ;{ }^{13} \mathrm{C}$ NMR $\left(100 \mathrm{MHz}, \mathrm{CDCl}_{3}\right) \delta=188.1,159.6,152.5,130.5,127.7,127.1,125.7$, 123.1, 120.4, 118.9, 114.0, 30.3, 21.6, 20.4 ppm; HRMS (ESI+): $m / z$ calcd for $\mathrm{C}_{14} \mathrm{H}_{12} \mathrm{BrO}_{2}$ [M $+\mathrm{H}]^{+}$291.0015, found 291.0008.

1,2,3,3-tetramethyl-3H-indol-1-ium iodide (2). To 2,3,3-trimethyl-3H-indole (1 mL, 6.2 mmol) in $\mathrm{CH}_{3} \mathrm{CN}(5 \mathrm{~mL})$ was added iodomethane $(0.44 \mathrm{~mL}, 7.06 \mathrm{mmol})$ and the solution was refluxed at $100{ }^{\circ} \mathrm{C}$ for $4 \mathrm{~h}$. The reaction mixture was filtered and the precipitate was washed with diethyl ether $(20 \mathrm{~mL}$ x 2$)$ and dried for $18 \mathrm{~h}$ to afford indolium iodide (5) as a light pink solid (1.77 g, 95\%). ${ }^{1} \mathrm{H}$ NMR (400 MHz, DMSO- $\left.d_{6}\right) \delta$ 7.94-7.85 (m, 1H), 7.85-7.77 (m, 1H), 7.66-7.56 (m, 2H), $3.96(\mathrm{~s}, 3 \mathrm{H}), 2.75(\mathrm{~s}, 3 \mathrm{H}), 1.52(\mathrm{~s}, 6 \mathrm{H}) ;{ }^{13} \mathrm{C}$ NMR (100 MHz, DMSO-d $) \delta$ $=196.0,142.0,141.6,129.3,128.8,123.2,115.1,53.9,34.6,21.7,13.9$ ppm. 
Table S1. Screening conditions for the Buchwald-Hartwig amination.

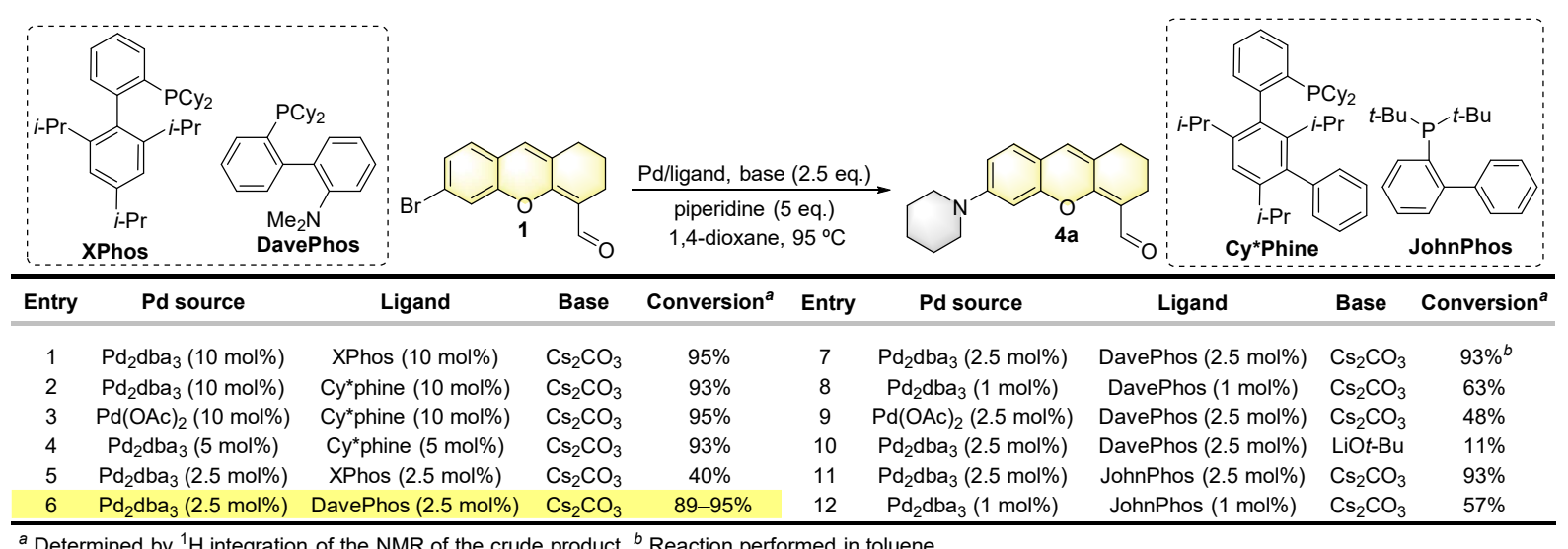

${ }^{a}$ Determined by ${ }^{1} \mathrm{H}$ integration of the NMR of the crude product. ${ }^{b}$ Reaction performed in toluene.

General procedure for NIR dyes 5. To 6-bromo-2,3-dihydro-1H-xanthene-4-carbaldehyde (1) $(20 \mathrm{mg}, 0.069 \mathrm{mmol})$ in anhydrous 1 ,4-dioxane $(1 \mathrm{~mL})$ under argon in a sealed tube were added the respective amine (5 eq.), $\mathrm{Pd}_{2}(\mathrm{dba})_{3}(2.5 \mathrm{~mol} \%)$, DavePhos $(2.5 \mathrm{~mol} \%)$ and $\mathrm{Cs}_{2} \mathrm{CO}_{3}$ (2.5 eq.). The mixture was heated at $95{ }^{\circ} \mathrm{C}$ for $18 \mathrm{~h}$ to reveal an intense orange spot (TLC EtOAc/hexane $1: 1, \mathrm{v} / \mathrm{v})$. The crude mixture 4 was concentrated in vacuo and directly dissolved in anhydrous $\mathrm{Ac}_{2} \mathrm{O}(4 \mathrm{~mL}) . \mathrm{K}_{2} \mathrm{CO}_{3}(25 \mathrm{mg}, 2.6$ eq.) and 1,2,3,3-tetramethyl-3Hindol-1-ium iodide (2) (25 mg, 1.2 eq.) were added and the mixture was stirred at $25^{\circ} \mathrm{C}$ for 18 $h$ to reveal an intense green spot (TLC $\mathrm{MeOH} / \mathrm{CH}_{2} \mathrm{Cl}_{2} 1: 9$, v/v). The medium was concentrated and the resulting residue dissolved in $\mathrm{CH}_{2} \mathrm{Cl}_{2}(20 \mathrm{~mL})$ and washed with $\mathrm{H}_{2} \mathrm{O}(10$ $\mathrm{mL}$ ). The organic layer was dried over anhydrous $\mathrm{Na}_{2} \mathrm{SO}_{4}$, filtered and concentrated in vacuo. Purification on silica gel (step gradient of $\mathrm{MeOH}$ in $\mathrm{CH}_{2} \mathrm{Cl}_{2}$ from $0 \%$ to $3 \%$ ) afforded NIR fluorophores 5 .

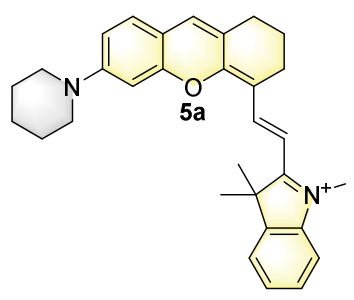

5a: $48 \%$ yield; Dark green solid; $\mathrm{R}_{\mathrm{f}}=0.5$ (silica gel, $\mathrm{MeOH} / \mathrm{CH}_{2} \mathrm{Cl}_{2} 1: 9$, v/v); IR (film) $v_{\max }$ $=3427,2933,1627,1552,1506,1445,1363,1311.1291,1271,1218,1196,1167,1107$, 
$1065,1018,926,800 \mathrm{~cm}^{-1} ;{ }^{1} \mathrm{H}$ NMR $\left(400 \mathrm{MHz}, \mathrm{CD}_{3} \mathrm{OD}\right) \delta=8.65(\mathrm{~d}, J=14.4 \mathrm{~Hz}, 1 \mathrm{H}), 7.60$ $7.30(\mathrm{~m}, 6 \mathrm{H}), 7.05(\mathrm{dd}, J=9.0,2.5 \mathrm{~Hz}, 1 \mathrm{H}), 6.89(\mathrm{~d}, J=2.4 \mathrm{~Hz}, 1 \mathrm{H}), 6.27(\mathrm{~d}, J=14.5 \mathrm{~Hz}$, $1 \mathrm{H}), 3.73(\mathrm{~s}, 3 \mathrm{H}), 3.57-3.50(\mathrm{~m}, 4 \mathrm{H}), 2.75(\mathrm{dt}, J=23.5,6.1 \mathrm{~Hz}, 4 \mathrm{H}), 1.97-1.90(\mathrm{~m}, 2 \mathrm{H}), 1.80$ $(\mathrm{s}, 6 \mathrm{H}) ;{ }^{13} \mathrm{C} \mathrm{NMR}\left(100 \mathrm{MHz}, \mathrm{CD}_{3} \mathrm{OD}\right): \delta=176.8,164.5,157.4,156.0,144.5,144.1,142.7$, $138.4,130.4,129.9,127.0,125.6,123.4,116.0,115.1,114.9,112.5,101.9,99.4,51.049 .7$, 32.1, 29.8, 28.5, 26.7, 25.4, 21.9 ppm; HRMS (ESI+): $m / z$ calcd for $\mathrm{C}_{31} \mathrm{H}_{35} \mathrm{~N}_{2} \mathrm{O}^{+}[\mathrm{M}]^{+\bullet}$ 451.2744, found 451.2758 .

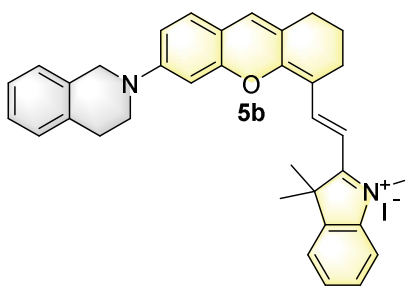

5b: 59\% yield; Dark green solid; $\mathrm{R}_{\mathrm{f}}=0.5$ (silica gel, $\mathrm{MeOH} / \mathrm{CH}_{2} \mathrm{Cl}_{2} 1: 9$, v/v); IR (film) $v_{\max }$ $=3437,2927,2856,1627,1559,1508,1447,1363,1292,1267,1219,1166,1152,1108$, $1065,1043,945,802 \mathrm{~cm}^{-1} ;{ }^{1} \mathrm{H}$ NMR $\left(400 \mathrm{MHz}, \mathrm{CD}_{3} \mathrm{OD}\right) \delta=8.68(\mathrm{~d}, J=14.4 \mathrm{~Hz}, 1 \mathrm{H}), 7.62-$ $7.20(\mathrm{~m}, 10 \mathrm{H}), 7.12(\mathrm{dd}, J=8.9,2.5 \mathrm{~Hz}, 1 \mathrm{H}), 6.95(\mathrm{~d}, J=2.4 \mathrm{~Hz}, 1 \mathrm{H}), 6.28(\mathrm{~d}, J=14.5 \mathrm{~Hz}$ 1H), $4.66(\mathrm{~s}, 2 \mathrm{H}), 3.78(\mathrm{t}, J=5.9 \mathrm{~Hz}, 2 \mathrm{H}), 3.73(\mathrm{~s}, 3 \mathrm{H}), 3.05(\mathrm{t}, J=5.9 \mathrm{~Hz}, 2 \mathrm{H}), 2.75(\mathrm{dt}, J=$ 24.4, 6.0 Hz, 4H), 2.03-1.89 (m, 2H), 1.82 (s, 6H).; $\left.{ }^{13} \mathrm{C} \mathrm{NMR} \mathrm{(100} \mathrm{MHz,} \mathrm{CD}_{3} \mathrm{OD}\right): \delta=176.9$, $164.5,157.2,155.1,144.7,144.1,142.7,138.5,136.4,134.7,130.4,129.9,129.1,128.1$, $127.6,127.6,127.1,125.6,123.4,116.0,115.1,114.0,112.5,106.4,102.0,98.7,51.0,49.9$, 46.4, 32.1, 29.9, 29.9, 28.5, 25.4, 21.9 ppm; HRMS (ESI+): $m / z$ calcd for $\mathrm{C}_{35} \mathrm{H}_{35} \mathrm{~N}_{2} \mathrm{O}^{+}[\mathrm{M}]^{+}$ 499.2744, found 499.2762.

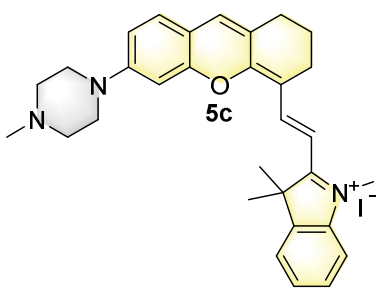


5c: $71 \%$ yield; Dark green solid; $\mathrm{R}_{\mathrm{f}}=0.5$ (silica gel, $\mathrm{MeOH} / \mathrm{CH}_{2} \mathrm{Cl}_{2} 1: 9$, v/v); IR (film) $v_{\max }$ $=3445,2928,2851,1626,1559,1508,1446,1364,1313,1292,1272,1220,1197,1168$, 1108, 1065, 1043, 1019, 926, $803 \mathrm{~cm}^{-1} ;{ }^{1} \mathrm{H}$ NMR (400 MHz, $\left.\mathrm{CD}_{3} \mathrm{OD}\right) \delta=8.71(\mathrm{~d}, J=14.6$ $\mathrm{Hz}, 1 \mathrm{H}), 7.62-7.35(\mathrm{~m}, 6 \mathrm{H}), 7.07(\mathrm{dd}, J=8.9,2.4 \mathrm{~Hz}, 1 \mathrm{H}), 6.97(\mathrm{~d}, J=2.1 \mathrm{~Hz}, 1 \mathrm{H}), 6.36(\mathrm{~d}$, $J=14.6 \mathrm{~Hz}, 1 \mathrm{H}), 3.78(\mathrm{~s}, 3 \mathrm{H}), 3.58-3.51(\mathrm{~m}, 4 \mathrm{H}), 2.83-2.69(\mathrm{~m}, 8 \mathrm{H}), 2.44(\mathrm{~s}, 3 \mathrm{H}), 1.98-1.89$ $(\mathrm{m}, 2 \mathrm{H}), 1.82(\mathrm{~s}, 6 \mathrm{H}) ;{ }^{13} \mathrm{C} \mathrm{NMR}\left(100 \mathrm{MHz}, \mathrm{CD}_{3} \mathrm{OD}\right): \delta=178.0,164.1,156.8,155.5,145.6$, $144.0,143.0,137.1,130.2,130.0,127.5,126.8,123.5,115.9,115.7,114.7,112.9,103.1$, 100.6, 55.5, 51.4, 47.9, 45.9, 32.4, 30.0, 28.4, 25.3, 21.8 ppm; HRMS (ESI+): $m / z$ calcd for $\mathrm{C}_{31} \mathrm{H}_{36} \mathrm{~N}_{3} \mathrm{O}^{+}[\mathrm{M}]^{+\bullet} 466.2853$, found 466.2858.

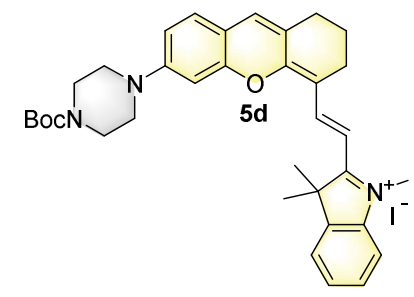

5d: $39 \%$ yield; Dark green solid; $\mathrm{R}_{\mathrm{f}}=0.5$ (silica gel, $\mathrm{MeOH} / \mathrm{CH}_{2} \mathrm{Cl}_{2} 1: 9$, v/v); IR (film) $v_{\max }$ $=3426,2927,2857,1688,1626,1559,1508,1446,1364,1311,1291,1271,1217,1167$, $1109 \mathrm{~m} 1066,1042,926,801 \mathrm{~cm}^{-1} ;{ }^{1} \mathrm{H}$ NMR $\left(400 \mathrm{MHz}, \mathrm{CD}_{3} \mathrm{OD}\right) \delta=8.72(\mathrm{~d}, J=14.6 \mathrm{~Hz}$ 1H), 7.63-7.35 (m, 6H), $7.07(\mathrm{dd}, J=8.9,2.4 \mathrm{~Hz}, 1 \mathrm{H}), 6.97(\mathrm{~d}, J=2.3 \mathrm{~Hz}, 1 \mathrm{H}), 6.36(\mathrm{~d}, J=$ $14.6 \mathrm{~Hz}, 1 \mathrm{H}), 3.78$ (s, 3H), $3.63(\mathrm{dd}, J=6.7,3.8 \mathrm{~Hz}, 4 \mathrm{H}), 3.51(\mathrm{dd}, J=6.6,3.9 \mathrm{~Hz}, 4 \mathrm{H}), 2.76$ $(\mathrm{dt}, J=22.9,6.0 \mathrm{~Hz}, 4 \mathrm{H}), 2.01-1.90(\mathrm{~m}, 2 \mathrm{H}), 1.82(\mathrm{~s}, 6 \mathrm{H}), 1.50(\mathrm{~s}, 9 \mathrm{H}) ;{ }^{13} \mathrm{C} \mathrm{NMR}(100 \mathrm{MHz}$, $\left.\mathrm{CD}_{3} \mathrm{OD}\right): \delta=177.9,164.1,156.8,156.4,155.6,145.6,144.0,143.0,137.2,130.2,130.0$, $127.5,126.7,123.5,115.9,115.7,114.7,112.9,103.0,100.5,81.7,64.3,51.3,48.2,32.3$, 30.0, 28.7, 28.4, 25.3, 21.8 ppm; HRMS (ESI+): $m / z$ calcd for $\mathrm{C}_{35} \mathrm{H}_{42} \mathrm{~N}_{3} \mathrm{O}_{3}{ }^{+}[\mathrm{M}]^{+\bullet} 552.3221$, found 552.3239. 


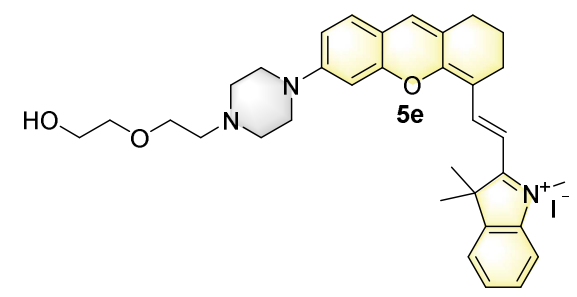

5e: $62 \%$ yield; Dark green solid; $\mathrm{R}_{\mathrm{f}}=0.5$ (silica gel, $\mathrm{MeOH} / \mathrm{CH}_{2} \mathrm{Cl}_{2} 1: 9$, v/v); IR (film) $v_{\max }$ $=3442,2931,2868,1734,1627,1560,1508,1447,1365,1312,1291,1271,1220,1197$, $1168,1109,1065,1044,1019,926,802 \mathrm{~cm}^{-1} ;{ }^{1} \mathrm{H}$ NMR $\left(400 \mathrm{MHz}, \mathrm{CD}_{3} \mathrm{OD}\right) \delta=8.70(\mathrm{~d}, J=$ $14.6 \mathrm{~Hz}, 1 \mathrm{H}), 7.61-7.33(\mathrm{~m}, 6 \mathrm{H}), 7.06(\mathrm{~d}, J=8.8 \mathrm{~Hz}, 1 \mathrm{H}), 6.94(\mathrm{~s}, 1 \mathrm{H}), 6.35$ (d, $J=14.6 \mathrm{~Hz}$, 1H), 4.28-4.19 (m, 2H), $3.78(\mathrm{~s}, 3 \mathrm{H}), 3.74-3.68(\mathrm{~m}, 4 \mathrm{H}), 3.57-3.48(\mathrm{~m}, 4 \mathrm{H}), 2.84-2.67$ (m, $10 \mathrm{H}), 1.98-1.88(\mathrm{~m}, 2 \mathrm{H}), 1.81(\mathrm{~s}, 6 \mathrm{H}) ;{ }^{13} \mathrm{C} \mathrm{NMR}\left(100 \mathrm{MHz}, \mathrm{CD}_{3} \mathrm{OD}\right): \delta=177.8,164.2$, $156.8,155.7,145.4,144.0,142.9,137.3,130.2,130.0,127.4,126.6,123.5,115.9,115.6$, $114.6,112.9,102.9,100.3,70.1,69.5,64.6,64.3,58.6,54.2,51.3,48.0,32.4,29.9,28.4,25.3$, 21.8 ppm; HRMS (ESI+): $m / z$ calcd for $\mathrm{C}_{34} \mathrm{H}_{42} \mathrm{~N}_{3} \mathrm{O}_{3}{ }^{+}[\mathrm{M}]^{+\bullet}$ 540.3221, found 540.3211.

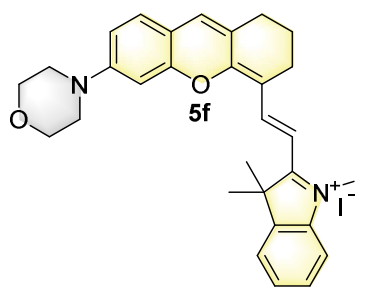

5f: $65 \%$ yield; Dark green solid; $\mathrm{R}_{\mathrm{f}}=0.5$ (silica gel, $\mathrm{MeOH} / \mathrm{CH}_{2} \mathrm{Cl}_{2} 1: 9$, v/v); IR (film) $v_{\max }$ $=3439,2926.2857,1626,1560,1508,1447,1364,1312,1290,1271,1221,1196,1168$, $1109,1066,1042,1019,926,801 \mathrm{~cm}^{-1} ;{ }^{1} \mathrm{H}$ NMR $\left(400 \mathrm{MHz}, \mathrm{CD}_{3} \mathrm{OD}\right) \delta=8.70(\mathrm{~d}, J=14.6$ $\mathrm{Hz}, 1 \mathrm{H}), 7.61-7.34(\mathrm{~m}, 6 \mathrm{H}), 7.05(\mathrm{dd}, J=8.8,2.5 \mathrm{~Hz}, 1 \mathrm{H}), 6.95(\mathrm{~d}, J=2.4 \mathrm{~Hz}, 1 \mathrm{H}), 6.36(\mathrm{~d}$, $J=14.6 \mathrm{~Hz}, 1 \mathrm{H}), 3.86(\mathrm{t}, J=4.9 \mathrm{~Hz}, 4 \mathrm{H}), 3.78(\mathrm{~s}, 3 \mathrm{H}), 3.47-3.42(\mathrm{~m}, 4 \mathrm{H}), 2.80-2.70(\mathrm{~m}$, 4H), 1.97-1.90 (t, 2H), $1.81(\mathrm{~s}, 6 \mathrm{H}) ;{ }^{13} \mathrm{C} \mathrm{NMR}\left(100 \mathrm{MHz}, \mathrm{CD}_{3} \mathrm{OD}\right): \delta=178.0,164.1,156.8$, $156.0,145.6,144.0,143.0,137.2,130.1,130.0,127.5,126.8,123.5,115.9,115.8,114.3$, 113.0, 103.0, 100.2, 67.6, 51.4, 48.7, 32.4, 30.0, 28.4, 25.3, 21.8 ppm; HRMS (ESI+): $m / z$ calcd for $\mathrm{C}_{30} \mathrm{H}_{33} \mathrm{~N}_{2} \mathrm{O}_{2}{ }^{+}[\mathrm{M}]^{+\bullet} 453.2537$, found 453.2550 . 


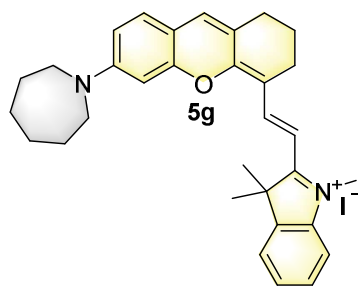

5g: 52\% yield; Dark green solid; $\mathrm{R}_{\mathrm{f}}=0.5$ (silica gel, $\mathrm{MeOH} / \mathrm{CH}_{2} \mathrm{Cl}_{2} 1: 9$, v/v); IR (film) v $v_{\max }$ $=3438,2927,2855,1627,1547,1507,1445,1309,1251,1228,1148,1106,1064,1043,927$, $802 \mathrm{~cm}^{-1} ;{ }^{1} \mathrm{H}$ NMR (400 MHz, $\left.\mathrm{CD}_{3} \mathrm{OD}\right) \delta=8.53(\mathrm{~d}, J=14.2 \mathrm{~Hz}, 1 \mathrm{H}), 7.47-7.17(\mathrm{~m}, 6 \mathrm{H})$, $6.86(\mathrm{~d}, J=11.4 \mathrm{~Hz}, 1 \mathrm{H}), 6.64(\mathrm{~s}, 1 \mathrm{H}), 6.12(\mathrm{~d}, J=14.3 \mathrm{~Hz}, 1 \mathrm{H}), 3.59(\mathrm{~m}, 7 \mathrm{H}), 2.65(\mathrm{dt}, J=$ 24.5, $6.1 \mathrm{~Hz}, 4 \mathrm{H}), 1.88-1.74(\mathrm{~m}, J=18.8,5.7 \mathrm{~Hz}, 6 \mathrm{H}), 1.69(\mathrm{~s}, 6 \mathrm{H}), 1.57-1.47(\mathrm{~m}, 4 \mathrm{H}) . ;{ }^{13} \mathrm{C}$ NMR (100 MHz, CD $\left.{ }_{3} \mathrm{OD}\right): \delta=175.9,164.8,157.7,154.8,144.2,143.8,142.4,139.5,130.8$, $129.9,126.6,124.6,123.4,116.1,114.6,113.4,112.1,101.1,96.8,51.0,50.7,31.9,29.7$, 28.6, 28.2, 27.8, 25.4, 22.0 ppm; HRMS (ESI+): $m / z$ calcd for $\mathrm{C}_{32} \mathrm{H}_{37} \mathrm{~N}_{2} \mathrm{O}^{+}[\mathrm{M}]^{+\bullet} 465.2900$, found 465.2913 .

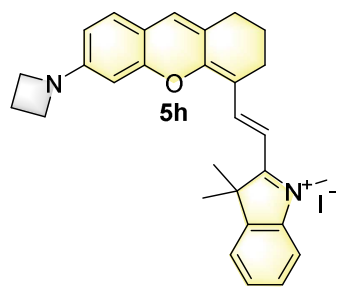

5h: 69\% yield; Dark green solid; $\mathrm{R}_{\mathrm{f}}=0.5$ (silica gel, $\mathrm{MeOH} / \mathrm{CH}_{2} \mathrm{Cl}_{2} 1: 9$, v/v); IR (film) v $\max$ $=3443,2926,2869,1627,1549,1508,1450,1361,1293,1256,1227,1163,1145,1105$, $1064,1043,925,806 \mathrm{~cm}^{-1} ;{ }^{1} \mathrm{H}$ NMR $\left(400 \mathrm{MHz}, \mathrm{CD}_{3} \mathrm{OD}\right) \delta=8.62(\mathrm{~d}, J=14.4 \mathrm{~Hz}, 1 \mathrm{H}), 7.61-$ $7.27(\mathrm{~m}, 6 \mathrm{H}), 6.53(\mathrm{~d}, J=10.8 \mathrm{~Hz}, 1 \mathrm{H}), 6.37(\mathrm{~s}, 1 \mathrm{H}), 6.24(\mathrm{~d}, J=14.4 \mathrm{~Hz}, 1 \mathrm{H}), 4.15(\mathrm{t}, J=$ $7.5 \mathrm{~Hz}, 4 \mathrm{H}), 3.70(\mathrm{~s}, 3 \mathrm{H}), 2.75(\mathrm{dt}, J=24.2,5.9 \mathrm{~Hz}, 4 \mathrm{H}), 2.51(\mathrm{~m}, J=7.5 \mathrm{~Hz}, 2 \mathrm{H}), 2.04-1.88$ (m, 2H), 1.79 (s, 6H); ${ }^{13} \mathrm{C}$ NMR (100 MHz, CD $\left.{ }_{3} \mathrm{OD}\right): \delta=176.3,164.6,157.3,156.1,144.2$, $144.0,142.5,139.5,130.7,129.9,126.8,124.8,123.4,116.0,115.0,112.3,111.9,101.4,95.6$, 
52.7, 50.8, 31.9, 29.8, 28.6, 25.4, 21.9, $17.1 \mathrm{ppm}$; HRMS (ESI+): $\mathrm{m} / z$ calcd for $\mathrm{C}_{29} \mathrm{H}_{31} \mathrm{~N}_{2} \mathrm{O}^{+}$ $[\mathrm{M}]^{+\bullet}$ 423.2431, found 423.2439 .

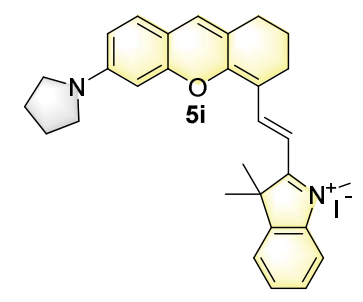

5i: $52 \%$ yield; Dark green solid; $\mathrm{R}_{\mathrm{f}}=0.5$ (silica gel, $\mathrm{MeOH} / \mathrm{CH}_{2} \mathrm{Cl}_{2} 1: 9, \mathrm{v} / \mathrm{v}$ ); IR (film) $v_{\max }$ $=3421,2927,2865,1629,1546,1508,1448,1360,1304,1293,1259,1225,1149,1106$, 1065, 1043, 1019, 923, $802 \mathrm{~cm}^{-1} ;{ }^{1} \mathrm{H}$ NMR (400 MHz, CD $\left.3 \mathrm{OD}\right) \delta=8.61(\mathrm{~d}, J=14.3 \mathrm{~Hz}, 1 \mathrm{H})$, $7.60-7.26(\mathrm{~m}, 6 \mathrm{H}), 6.81(\mathrm{dd}, J=8.9,2.3 \mathrm{~Hz}, 1 \mathrm{H}), 6.57(\mathrm{~d}, J=2.2 \mathrm{~Hz}, 1 \mathrm{H}), 6.20(\mathrm{~d}, J=14.3$ $\mathrm{Hz}, 1 \mathrm{H}), 3.68$ (s, 3H), 3.53-3.47 (m, 4H), $2.76(\mathrm{dt}, J=26.2,6.1 \mathrm{~Hz}, 4 \mathrm{H}), 1.99-1.90(\mathrm{~m}, 2 \mathrm{H})$, $1.79(\mathrm{~s}, 6 \mathrm{H}) ;{ }^{13} \mathrm{C}$ NMR $\left(100 \mathrm{MHz}, \mathrm{CD}_{3} \mathrm{OD}\right): \delta=175.7,164.8,157.5,153.4,144.3,143.5$, $142.4,139.9,130.7,129.9,126.5,124.3,123.4,116.1,114.7,114.2,112.1,100.9,97.1,64.3$, 50.7, 31.8, 29.7, 28.7, 26.4, 25.4, 22.0 ppm; HRMS (ESI+): $m / z$ calcd for $\mathrm{C}_{30} \mathrm{H}_{33} \mathrm{~N}_{2} \mathrm{O}^{+}[\mathrm{M}]^{+}$ 437.2587, found 437.2597.

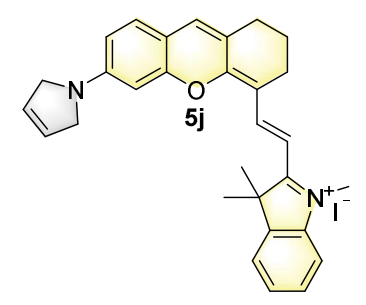

Adapting the general procedure for NIR dyes 5, to 6-bromo-2,3-dihydro-1H-xanthene-4carbaldehyde (1) in anhydrous 1,4-dioxane under argon in a sealed tube were added diallylamine (5 eq.), $\mathrm{Pd}_{2}(\mathrm{dba})_{3}\left(2.5 \mathrm{~mol} \%\right.$ ), DavePhos (2.5 mol\%) and $\mathrm{Cs}_{2} \mathrm{CO}_{3}$ (2.5 eq.). The crude mixture $4 \mathbf{n}$ was concentrated in vacuo and after addition of dichloromethane $(5 \mathrm{~mL})$ Grubbs' $1^{\text {st }}$ generation catalyst $(10 \mathrm{mg}, 20 \mathrm{~mol} \%)$ and 1,4-Benzoquinone (6 mg, 0.8 eq.) were added and the solution was stirred for $2 \mathrm{~h}$. The mixture was then filtered using a silica plug 
and the filtrate was concentrated in vacuo. The crude mixture was dissolved in anhydrous $\mathrm{Ac}_{2} \mathrm{O}$ (4 mL), $\mathrm{K}_{2} \mathrm{CO}_{3}(25 \mathrm{mg}, 2.6$ eq.) and 1,2,3,3-tetramethyl-3H-indol-1-ium iodide (2) (25 $\mathrm{mg}, 1.2$ eq.). The purification of dye $\mathbf{5} \mathbf{j}$ was performed according to the general procedure.

5j: $10 \%$ yield over 3 steps; Dark blue solid; $\mathrm{R}_{\mathrm{f}}=0.5$ (silica gel, $\mathrm{MeOH} / \mathrm{CH}_{2} \mathrm{Cl}_{2} 1: 9$, v/v); IR (film) $v_{\max }=3404,2925,2853,1635,1550,1508,1447,1359,1292,1258,1226,1159,1147$, 1104, 1064, 1043, 1019, $925 \mathrm{~cm}^{-1} ;{ }^{1} \mathrm{H}$ NMR (400 MHz, CD $\left.{ }_{3} \mathrm{OD}\right) \delta 8.66(\mathrm{~d}, J=14.3 \mathrm{~Hz}, 1 \mathrm{H})$, 7.59-7.28 (m, 6H), 6.79 (dd, $J=8.8,2.3 \mathrm{~Hz}, 1 \mathrm{H}), 6.62-6.57(\mathrm{~m}, 1 \mathrm{H}), 6.26(\mathrm{~d}, J=14.4 \mathrm{~Hz}$, 1H), $6.07(\mathrm{~s}, 2 \mathrm{H}), 4.31(\mathrm{~s}, 4 \mathrm{H}), 3.71(\mathrm{~s}, 3 \mathrm{H}), 2.77(\mathrm{dt}, J=25.5,6.1 \mathrm{~Hz}, 5 \mathrm{H}), 2.01-1.89(\mathrm{~m}$, 2H), 1.80 (s, 6H). ${ }^{13} \mathrm{C}$ NMR (100 MHz, CD $\left.3 \mathrm{OD}\right) \delta=176.4,164.8,157.0,152.7,144.2,142.6$, $139.5,130.9,129.9,126.9,126.8,124.8,123.4,116.0,114.7,113.6,112.3,101.4,97.2,55.9$, 50.8, 31.9, 30.7, 29.8, 28.6, 25.4, 21.9 ppm; HRMS (ESI+): $m / z$ calcd for $\mathrm{C}_{30} \mathrm{H}_{31} \mathrm{~N}_{2} \mathrm{O}^{+}[\mathrm{M}]^{+\bullet}$ 435.2431, found 435.2427 .

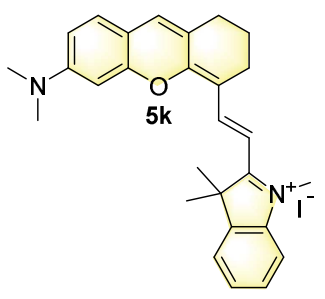

5k: 48\% yield; Dark green solid; $\mathrm{R}_{\mathrm{f}}=0.5$ (silica gel, $\mathrm{MeOH} / \mathrm{CH}_{2} \mathrm{Cl}_{2} 1: 9$, v/v); IR (film) $v_{\max }$ $=3447,2927,2856,1630,1552,1510,1443,1358,1308,1262,1170,1150,1107,1065$, 1043, 922, $803 \mathrm{~cm}^{-1} ;{ }^{1} \mathrm{H}$ NMR (400 MHz, CD $\left.{ }_{3} \mathrm{OD}\right) \delta=\delta 8.64(\mathrm{~d}, J=14.3 \mathrm{~Hz}, 1 \mathrm{H}), 7.59-7.22$ $(\mathrm{m}, 6 \mathrm{H}), 6.92(\mathrm{dd}, J=8.9,2.4 \mathrm{~Hz}, 1 \mathrm{H}), 6.70(\mathrm{dd}, J=2.4,0.7 \mathrm{~Hz}, 1 \mathrm{H}), 6.25(\mathrm{~d}, J=14.3 \mathrm{~Hz}$, 1H), $3.71(\mathrm{~s}, 3 \mathrm{H}), 3.18(\mathrm{~s}, 6 \mathrm{H}), 2.75(\mathrm{dt}, J=25.1,6.0 \mathrm{~Hz}, 4 \mathrm{H}), 2.00-1.89$ (m, 2H), 1.79 (s, $6 \mathrm{H}) ;{ }^{13} \mathrm{C}$ NMR $\left(100 \mathrm{MHz}, \mathrm{CD}_{3} \mathrm{OD}\right): \delta=176.4,164.7,157.3,155.7,144.2,142.5,139.2$, $130.5,129.9,126.8,124.9,123.4,116.0,114.5,113.4,112.3,106.4,101.5,97.3,50.8,40.5$, 32.0, 29.8, 28.6, 25.4, 21.9 ppm; HRMS (ESI+): $m / z$ calcd for $\mathrm{C}_{28} \mathrm{H}_{31} \mathrm{~N}_{2} \mathrm{O}^{+}[\mathrm{M}]^{+\bullet} 411.2431$, found 411.2441 . 


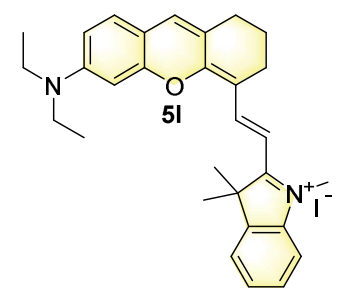

5l: 50\% yield; Dark green solid; $\mathrm{R}_{\mathrm{f}}=0.5$ (silica gel, $\mathrm{MeOH} / \mathrm{CH}_{2} \mathrm{Cl}_{2} 1: 9$, v/v); IR (film) $v_{\max }$ $=3447,2971,2930,1628,1548,1508,1444,1367,1312,1291,1252,1227,1166,1149$, $1107,1064,1043,1019,926,802 \mathrm{~cm}^{-1} ;{ }^{1} \mathrm{H}$ NMR $\left(400 \mathrm{MHz}, \mathrm{CD}_{3} \mathrm{OD}\right) \delta=\delta 8.63(\mathrm{~d}, J=14.3$ $\mathrm{Hz}, 1 \mathrm{H}), 7.68-7.22(\mathrm{~m}, 6 \mathrm{H}), 6.92(\mathrm{dd}, J=9.0,2.5 \mathrm{~Hz}, 1 \mathrm{H}), 6.69(\mathrm{~d}, J=2.4 \mathrm{~Hz}, 1 \mathrm{H}), 6.22(\mathrm{~d}$, $J=14.3 \mathrm{~Hz}, 1 \mathrm{H}), 3.70(\mathrm{~s}, 3 \mathrm{H}), 3.59(\mathrm{q}, J=7.1 \mathrm{~Hz}, 4 \mathrm{H}), 2.75(\mathrm{dt}, J=23.8,6.1 \mathrm{~Hz}, 4 \mathrm{H}), 2.12-$ $1.85(\mathrm{~m}, 2 \mathrm{H}), 1.79(\mathrm{~s}, 6 \mathrm{H}), 1.28(\mathrm{t}, J=7.1 \mathrm{~Hz}, 6 \mathrm{H}) ;{ }^{13} \mathrm{C} \mathrm{NMR}\left(100 \mathrm{MHz}, \mathrm{CD}_{3} \mathrm{OD}\right): \delta=175.9$, $164.8,157.7,153.7,144.2,143.8,142.4,139.5,130.8,129.8,126.6,124.5,123.4,116.1$, $114.5,113.5,112.1,101.1,96.8,50.7,46.0,31.9,29.7,28.6,25.4,22.0,12.8$ ppm; HRMS (ESI+): $m / z$ calcd for $\mathrm{C}_{30} \mathrm{H}_{35} \mathrm{~N}_{2} \mathrm{O}^{+}[\mathrm{M}]^{+\bullet} 439.2744$, found 439.2755 .

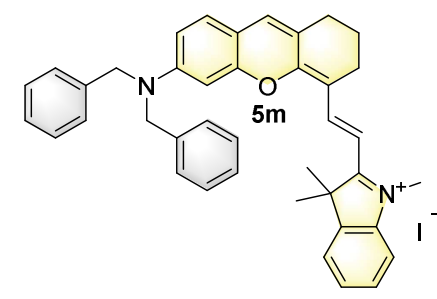

5m: $28 \%$ yield; Dark green solid; $\mathrm{R}_{\mathrm{f}}=0.5$ (silica gel, $\mathrm{MeOH} / \mathrm{CH}_{2} \mathrm{Cl}_{2} 1: 9$, v/v); IR (film) $v_{\max }=3409,2926,2856,1629,1556,1508,1447,1368,1291,1270,1222,1167,1109,1063$, 1043, 927, $804 \mathrm{~cm}^{-1} ;{ }^{1} \mathrm{H}$ NMR (400 MHz, CD $\left.3 \mathrm{OD}\right) \delta=8.54(\mathrm{~d}, J=14.5 \mathrm{~Hz}, 1 \mathrm{H}), 7.64-7.27$ $(\mathrm{m}, 16 \mathrm{H}), 6.95(\mathrm{dd}, J=8.9,2.5 \mathrm{~Hz}, 1 \mathrm{H}), 6.68(\mathrm{~d}, J=2.4 \mathrm{~Hz}, 1 \mathrm{H}), 6.26(\mathrm{~d}, J=14.5 \mathrm{~Hz}, 1 \mathrm{H})$, $4.94(\mathrm{~s}, 4 \mathrm{H}), 3.70(\mathrm{~s}, 3 \mathrm{H}), 2.71(\mathrm{dt}, J=28.8,6.1 \mathrm{~Hz}, 4 \mathrm{H}), 1.97-1.85(\mathrm{~m}, 2 \mathrm{H}), 1.69(\mathrm{~s}, 6 \mathrm{H}) ;{ }^{13} \mathrm{C}$ NMR $\left(100 \mathrm{MHz}, \mathrm{CD}_{3} \mathrm{OD}\right): \delta=177.1,164.4,156.7,154.4,145.0,144.0,142.6,138.8,138.2$, $130.2,130.0,130.0,128.5,127.7,127.1,125.6,123.4,115.8,114.8,113.9,112.6,102.1,99.2$, 
56.6, 51.0, 32.0, 29.7, 28.6, 25.1, 21.8 ppm; HRMS (ESI+): $m / z$ calcd for $\mathrm{C}_{40} \mathrm{H}_{39} \mathrm{~N}_{2} \mathrm{O}^{+}[\mathrm{M}]^{+}$ 563.3057, found 563.3059.

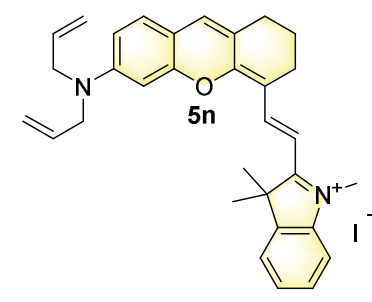

5n: $35 \%$ yield; Dark green solid; $\mathrm{R}_{\mathrm{f}}=0.5$ (silica gel, $\mathrm{MeOH} / \mathrm{CH}_{2} \mathrm{Cl}_{2} 1: 9$, v/v); IR (film) $v_{\max }$ $=3419,2919,2846,1627,1556,1509,1447,1366,1309,1291,1270,1223,1166,1109,927$, $804 \mathrm{~cm}^{-1} ;{ }^{1} \mathrm{H}$ NMR $\left(400 \mathrm{MHz}, \mathrm{CD}_{3} \mathrm{OD}\right) \delta=8.66(\mathrm{~d}, J=14.7 \mathrm{~Hz}, 1 \mathrm{H}), 7.60-7.31(\mathrm{~m}, 6 \mathrm{H})$, $6.89(\mathrm{dd}, J=8.9,2.5 \mathrm{~Hz}, 1 \mathrm{H}), 6.76(\mathrm{~d}, J=2.4 \mathrm{~Hz}, 1 \mathrm{H}), 6.29(\mathrm{~d}, J=14.4 \mathrm{~Hz}, 1 \mathrm{H}), 5.95(\mathrm{dd}, J$ $=17.1,10.5 \mathrm{~Hz}, 2 \mathrm{H}), 5.30-5.22(\mathrm{~m}, 4 \mathrm{H}), 4.18(\mathrm{dd}, J=4.8,1.8 \mathrm{~Hz}, 4 \mathrm{H}), 3.72(\mathrm{~s}, 3 \mathrm{H}), 2.76(\mathrm{dt}$, $J=25.8,5.9 \mathrm{~Hz}, 4 \mathrm{H}), 1.94(\mathrm{p}, J=6.3 \mathrm{~Hz}, 2 \mathrm{H}), 1.79(\mathrm{~s}, 6 \mathrm{H}) .{ }^{13} \mathrm{C} \mathrm{NMR}\left(100 \mathrm{MHz}, \mathrm{CD}_{3} \mathrm{OD}\right) \delta$ $=176.8,164.7,157.1,154.5,144.7,144.1,142.6,138.7,134.0,130.4,129.9,127.0,125.3$, $123.4,117.2,115.9,114.7,113.7,112.4,101.8,98.2,54.4,50.9,32.0,29.8,28.5,25.2,21.9$ ppm; HRMS (ESI+): $m / z$ calcd for $\mathrm{C}_{32} \mathrm{H}_{35} \mathrm{~N}_{2} \mathrm{O}^{+}[\mathrm{M}]^{+\bullet} 463.2744$, found 463.2738.

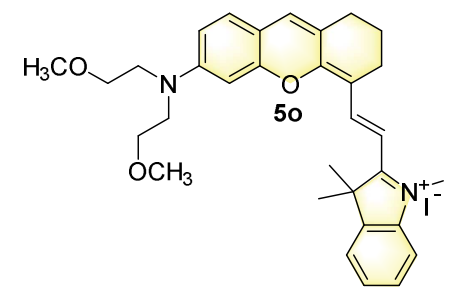

5o: $33 \%$ yield; Dark green solid; $\mathrm{R}_{\mathrm{f}}=0.5$ (silica gel, $\mathrm{MeOH} / \mathrm{CH}_{2} \mathrm{Cl}_{2} 1: 9$, v/v); IR (film) $v_{\max }$ $=3438,2925,2855,1629,1553,1508,1448,1368,1312,1292,1226,1192,1167,1107,927$, $802 \mathrm{~cm}^{-1} ;{ }^{1} \mathrm{H}$ NMR $\left(400 \mathrm{MHz}, \mathrm{CD}_{3} \mathrm{OD}\right) \delta=8.67(\mathrm{~d}, J=14.4 \mathrm{~Hz}, 1 \mathrm{H}), 7.59-7.30(\mathrm{~m}, 6 \mathrm{H})$, $6.97(\mathrm{dd}, J=8.9,2.5 \mathrm{~Hz}, 1 \mathrm{H}), 6.88(\mathrm{~d}, J=2.4 \mathrm{~Hz}, 1 \mathrm{H}), 6.27(\mathrm{~d}, J=14.4 \mathrm{~Hz}, 1 \mathrm{H}), 3.77(\mathrm{t}, J=$ $5.5 \mathrm{~Hz}, 4 \mathrm{H}), 3.72(\mathrm{~s}, 3 \mathrm{H}), 3.66(\mathrm{t}, J=5.5 \mathrm{~Hz}, 4 \mathrm{H}), 3.39(\mathrm{~s}, 6 \mathrm{H}), 2.76(\mathrm{dt}, J=24.1,6.1 \mathrm{~Hz}$, 4H), 2.01-1.90 (m, 2H), $\left.1.80(\mathrm{~s}, 6 \mathrm{H}) ;{ }^{13} \mathrm{C} \mathrm{NMR} \mathrm{(100} \mathrm{MHz,} \mathrm{CD}_{3} \mathrm{OD}\right): \delta=176.5,164.7,157.3$, 
$154.5,144.4,144.2,142.5,138.9,130.4,129.9,126.9,125.1,123.4,116.0,114.7,113.7$, 112.3, 101.6, 98.2, 71.4, 59.4, 52.3, 50.9, 32.0, 29.8, 28.6, 25.3, 21.9 ppm; HRMS (ESI+): $m / z$ calcd for $\mathrm{C}_{32} \mathrm{H}_{39} \mathrm{~N}_{2} \mathrm{O}_{3}{ }^{+}[\mathrm{M}]^{+\bullet} 499.2955$, found 499.2979 .

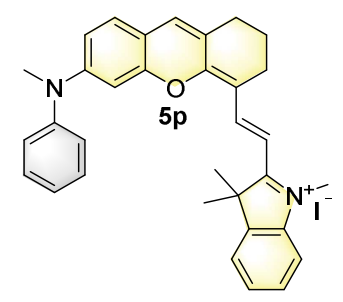

5p: $47 \%$ yield; Dark green solid; $\mathrm{R}_{\mathrm{f}}=0.5$ (silica gel, $\mathrm{MeOH} / \mathrm{CH}_{2} \mathrm{Cl}_{2} 1: 9$, v/v); IR (film) $v_{\max }$ $=3433,2923,2854,1628,1558,1493,1444,1369,1297,1269,1228,1168,1108,1065$, 1043, 927, $798 \mathrm{~cm}^{-1} ;{ }^{1} \mathrm{H}$ NMR (400 MHz, CD $\left.3 \mathrm{OD}\right) \delta=\delta 8.64(\mathrm{~d}, J=14.5 \mathrm{~Hz}, 1 \mathrm{H}), 7.62-7.24$ $(\mathrm{m}, 11 \mathrm{H}), 6.78(\mathrm{dd}, J=11.8,3.1 \mathrm{~Hz}, 2 \mathrm{H}), 6.33(\mathrm{~d}, J=14.5 \mathrm{~Hz}, 1 \mathrm{H}), 3.74(\mathrm{~s}, 3 \mathrm{H}), 3.48(\mathrm{~s}$, 3H), $2.75(\mathrm{dt}, J=25.0,5.9 \mathrm{~Hz}, 4 \mathrm{H}), 2.00-1.88(\mathrm{~m}, 2 \mathrm{H}), 1.74(\mathrm{~s}, 6 \mathrm{H}) ;{ }^{13} \mathrm{C} \mathrm{NMR}(100 \mathrm{MHz}$, $\left.\mathrm{CD}_{3} \mathrm{OD}\right): \delta=177.4,164.3,156.8,154.8,148.0,145.2,144.0,142.7,137.8,131.3,130.0$, $129.9,127.9,127.7,127.3,126.1,123.4,115.8,115.4,115.0,112.7,102.5,99.7,51.1,41.0$, 32.2, 29.9, 28.4, 25.3, 21.9 ppm; HRMS (ESI+): $m / z$ calcd for $\mathrm{C}_{33} \mathrm{H}_{33} \mathrm{~N}_{2} \mathrm{O}^{+}[\mathrm{M}]^{+\bullet} 473.2587$, found 473.2590 .

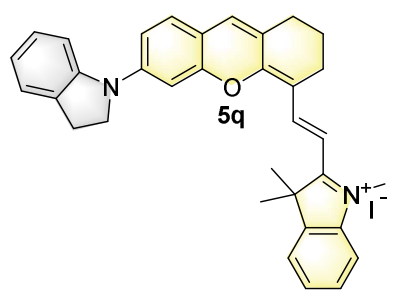

5q: $29 \%$ yield; Dark green solid; $\mathrm{R}_{\mathrm{f}}=0.5$ (silica gel, $\mathrm{MeOH} / \mathrm{CH}_{2} \mathrm{Cl}_{2} 1: 9$, v/v); IR (film) $v_{\max }$ $=3440,2925,2855,1627,1599,1559,1508,1448,1365,1291,1260,1226,1168,1110,927$, $802 \mathrm{~cm}^{-1} ;{ }^{1} \mathrm{H}$ NMR $\left(400 \mathrm{MHz}, \mathrm{CD}_{3} \mathrm{OD}\right) \delta=8.73(\mathrm{~d}, J=14.7 \mathrm{~Hz}, 1 \mathrm{H}), 7.64-7.15(\mathrm{~m}, 11 \mathrm{H})$, $6.92(\mathrm{td}, J=7.4,0.9 \mathrm{~Hz}, 1 \mathrm{H}), 6.41(\mathrm{~d}, J=14.7 \mathrm{~Hz}, 1 \mathrm{H}), 4.12(\mathrm{t}, J=8.3 \mathrm{~Hz}, 2 \mathrm{H}), 3.80(\mathrm{~s}, 3 \mathrm{H})$, $3.21(\mathrm{t}, J=8.3 \mathrm{~Hz}, 2 \mathrm{H}), 2.77(\mathrm{dt}, J=25.0,5.9 \mathrm{~Hz}, 4 \mathrm{H}), 2.02-1.91(\mathrm{~m}, 2 \mathrm{H}), 1.81(\mathrm{~s}, 6 \mathrm{H}) ;{ }^{13} \mathrm{C}$ 
NMR (100 MHz, $\left.\mathrm{CD}_{3} \mathrm{OD}\right): \delta=178.3,163.7,156.3,149.2,145.9,145.7,143.9,143.0,136.4$, $134.2,130.1,130.0,128.2,127.7,127.4,126.7,123.5,122.7,116.8,116.1,115.9,113.1$, 111.4, 103.6, 102.2, 53.4, 51.5, 32.5, 30.0, 28.9, 28.3, 25.3, 21.8 ppm; HRMS (ESI+): $m / z$ calcd for $\mathrm{C}_{34} \mathrm{H}_{33} \mathrm{~N}_{2} \mathrm{O}^{+}[\mathrm{M}]^{+\bullet} 485.2587$, found 485.2602.

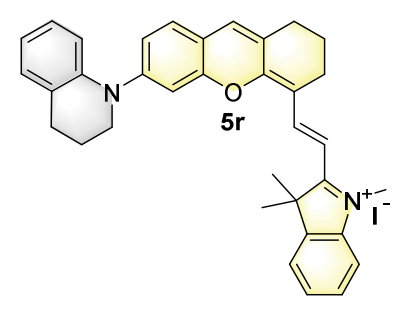

5r: $51 \%$ yield; Dark blue solid; $\mathrm{R}_{\mathrm{f}}=0.5$ (silica gel, $\mathrm{MeOH} / \mathrm{CH}_{2} \mathrm{Cl}_{2} 1: 9$, v/v); IR (film) $v_{\max }$ $=3430,2926,2855,1624,1560,1504,1490,1444,1366,1258,1226,1168,1109,1064$, 1043, 927, $801 \mathrm{~cm}^{-1} ;{ }^{1} \mathrm{H}$ NMR (400 MHz, CD $\left.3 \mathrm{OD}\right) \delta=8.68(\mathrm{~d}, J=14.8 \mathrm{~Hz}, 1 \mathrm{H}), 7.62-6.92$ (m, 12H), $6.41(\mathrm{~d}, J=14.7 \mathrm{~Hz}, 1 \mathrm{H}), 3.79(\mathrm{~d}, J=4.4 \mathrm{~Hz}, 5 \mathrm{H}), 2.85-2.66(\mathrm{~m}, 6 \mathrm{H}), 2.05(\mathrm{t}, J=$ $6.3 \mathrm{~Hz}, 2 \mathrm{H}), 1.95(\mathrm{~d}, J=6.0 \mathrm{~Hz}, 2 \mathrm{H}), 1.76(\mathrm{~s}, 6 \mathrm{H}) . ;{ }^{13} \mathrm{C} \mathrm{NMR}\left(100 \mathrm{MHz}, \mathrm{CD}_{3} \mathrm{OD}\right): \delta=178.4$, $163.6,156.2,153.5,146.0,143.9,143.0,142.3,136.2,132.1,130.4,130.0,129.6,127.8$, $127.7,127.3,123.8,123.5,121.4,118.8,117.4,115.8,113.2,105.3,103.8,51.5,50.3,32.6$, 30.0, 28.2, 25.3, 25.1, 21.8 ppm; HRMS (ESI+): $m / z$ calcd for $\mathrm{C}_{35} \mathrm{H}_{35} \mathrm{~N}_{2} \mathrm{O}^{+}[\mathrm{M}]^{+\bullet} 499.2744$, found 499.2760 .

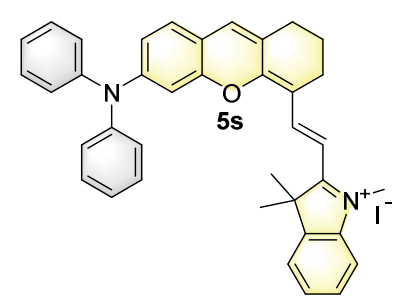

5s: $14 \%$ yield; Dark green solid; $\mathrm{R}_{\mathrm{f}}=0.5$ (silica gel, $\mathrm{MeOH} / \mathrm{CH}_{2} \mathrm{Cl}_{2} 1: 9, \mathrm{v} / \mathrm{v}$ ); IR (film) $v_{\max }$ $=3439,2927,2856,1626,1594,1562,1503,1490,1455,1414,1368,1260,1231,1186$, $1171,1110,1064,927,801 \mathrm{~cm}^{-1} ;{ }^{1} \mathrm{H}$ NMR $\left(400 \mathrm{MHz}, \mathrm{CD}_{3} \mathrm{OD}\right) \delta=8.57(\mathrm{~d}, J=14.8 \mathrm{~Hz}, 1 \mathrm{H})$, $7.59-7.17(\mathrm{~m}, 16 \mathrm{H}), 6.88(\mathrm{dd}, J=8.6,2.3 \mathrm{~Hz}, 1 \mathrm{H}), 6.82(\mathrm{dd}, J=2.2,0.7 \mathrm{~Hz}, 1 \mathrm{H}), 6.41(\mathrm{~d}, J=$ 
$14.8 \mathrm{~Hz}, 1 \mathrm{H}), 3.78(\mathrm{~s}, 3 \mathrm{H}), 2.75(\mathrm{dt}, J=29.3,5.9 \mathrm{~Hz}, 4 \mathrm{H}), 1.97-1.89(\mathrm{~m}, 2 \mathrm{H}), 1.65(\mathrm{~s}, 6 \mathrm{H})$; ${ }^{13} \mathrm{C}$ NMR (100 MHz, $\left.\mathrm{CD}_{3} \mathrm{OD}\right): \delta=178.7,163.4,155.9,153.4,147.3,146.2,143.8,143.0$, $135.8,131.0,130.1,129.8,128.0,127.9,127.5,126.8,123.5,119.2,117.4,115.8,113.3$, 105.9, 104.1, 51.5, 32.5, 30.0, 28.0, 25.2, 21.7 ppm; HRMS (ESI+): $m / z$ calcd for $\mathrm{C}_{38} \mathrm{H}_{35} \mathrm{~N}_{2} \mathrm{O}^{+}$ $[\mathrm{M}]^{+\bullet} 535.2744$, found 535.2763.

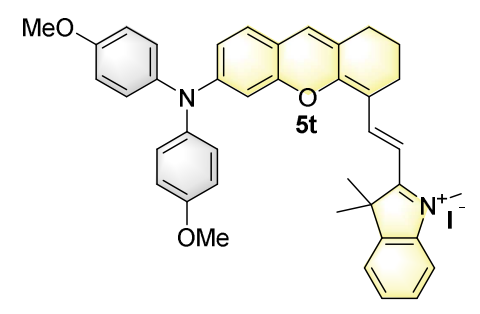

5t: $43 \%$ yield; Dark blue solid; $\mathrm{R}_{\mathrm{f}}=0.5$ (silica gel, $\mathrm{MeOH} / \mathrm{CH}_{2} \mathrm{Cl}_{2} 1: 9$, v/v); IR (film) $v_{\max }=$ 3432, 2931, 1626, 1560, 1499, 1445, 1413, 1368, 1291, 1260, 1243, 1229, 1168, 1107, 1064, $1042,1020,928,803 \mathrm{~cm}^{-1} ;{ }^{1} \mathrm{H}$ NMR $\left(400 \mathrm{MHz}, \mathrm{CD}_{3} \mathrm{OD}\right) \delta=8.52(\mathrm{~d}, J=14.6 \mathrm{~Hz}, 1 \mathrm{H}), 7.58-$ $7.29(\mathrm{~m}, 6 \mathrm{H}), 7.23-7.16(\mathrm{~m}, 4 \mathrm{H}), 7.04-6.96(\mathrm{~m}, 4 \mathrm{H}), 6.76(\mathrm{dd}, J=8.7,2.3 \mathrm{~Hz}, 1 \mathrm{H}), 6.63(\mathrm{dd}$, $J=2.2,0.8 \mathrm{~Hz}, 1 \mathrm{H}), 6.35(\mathrm{~d}, J=14.6 \mathrm{~Hz}, 1 \mathrm{H}), 3.85(\mathrm{~s}, 6 \mathrm{H}), 3.74(\mathrm{~s}, 3 \mathrm{H}), 2.73(\mathrm{dt}, J=27.0$, $6.1 \mathrm{~Hz}, 4 \mathrm{H}), 1.96-1.87(\mathrm{~m}, 2 \mathrm{H}), 1.64(\mathrm{~s}, 6 \mathrm{H}) ;{ }^{13} \mathrm{C} \mathrm{NMR}\left(100 \mathrm{MHz}, \mathrm{CD}_{3} \mathrm{OD}\right): \delta=177.8$, $163.9,159.5,156.2,154.6,145.5,143.9,142.7,139.7,136.9,130.0,129.9,129.2,127.6$, $126.8,123.4,117.3,116.4,116.3,115.8,113.0,103.2,103.0,56.2,51.2,32.4,29.9,28.2$, 25.3, 21.8 ppm; HRMS (ESI+): $m / z$ calcd for $\mathrm{C}_{40} \mathrm{H}_{39} \mathrm{~N}_{2} \mathrm{O}_{3}{ }^{+}[\mathrm{M}]^{+\bullet}$ 595.2955, found 595.2981.

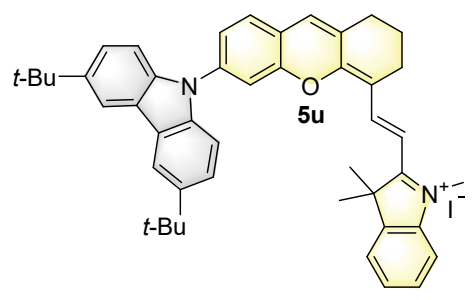

5u: $38 \%$ yield; Dark blue solid; $\mathrm{R}_{\mathrm{f}}=0.5$ (silica gel $\mathrm{MeOH} / \mathrm{CH}_{2} \mathrm{Cl}_{2} 1: 9$, v/v); IR (film) $v_{\max }=$ $3432,2960,2866,1610,1568,1535,1509,1496,1460,1365,1279,1189,1111,1064,923$, $803 \mathrm{~cm}^{-1} ;{ }^{1} \mathrm{H}$ NMR $\left(400 \mathrm{MHz}, \mathrm{CD}_{3} \mathrm{OD}\right) \delta=8.66(\mathrm{~d}, J=15.2 \mathrm{~Hz}, 1 \mathrm{H}), 8.13-8.08(\mathrm{~m}, 2 \mathrm{H})$, ), 
7.61-7.20 (m, 12H), $6.50(\mathrm{~d}, J=15.2 \mathrm{~Hz}, 1 \mathrm{H}), 3.80(\mathrm{~s}, 3 \mathrm{H}), 2.68(\mathrm{dd}, J=30.4,6.1 \mathrm{~Hz}, 4 \mathrm{H})$, 1.93-1.81 (m, 2H), 1.62 (s, 6H), $1.35(\mathrm{~s}, 18 \mathrm{H}) ;{ }^{13} \mathrm{C}$ NMR (100 MHz, CD $\left.{ }_{3} \mathrm{OD}\right): \delta=180.8$, $161.2,155.0,147.5,145.2,143.6,143.5,142.2,140.0,132.4,131.7,130.2,130.1,129.0$, $125.3,125.1,124.3,123.6,122.0,117.5,116.1,114.3,114.1,110.3,106.9,52.3,35.6,33.4$, 32.4, 30.4, 27.8, 25.1, 21.6 ppm; HRMS (ESI+): $m / z$ calcd for $\mathrm{C}_{46} \mathrm{H}_{49} \mathrm{~N}_{2} \mathrm{O}^{+}[\mathrm{M}]^{+\bullet}$ 645.3839, found 645.3854 .

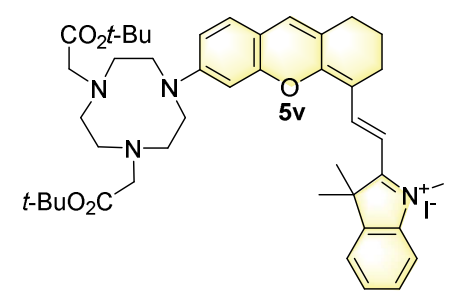

5v: $16 \%$ yield; Dark green solid; $\mathrm{R}_{\mathrm{f}}=0.5$ (silica gel, $\mathrm{MeOH} / \mathrm{CH}_{2} \mathrm{Cl}_{2} 1: 9, \mathrm{v} / \mathrm{v}$ ); IR (film) v $v_{\max }$ $=3419,2918,2846,1730,1628,1556,1509,1446,1368,1308,1291,1266,1222,1156$, 1109, 1065, 1043, 1018, $991 \mathrm{~cm}^{-1} ;{ }^{1} \mathrm{H}$ NMR (400 MHz, CD $\left.{ }_{3} \mathrm{OD}\right) \delta=8.66(\mathrm{~d}, J=14.3 \mathrm{~Hz}$, 1H), 7.57-7.28 (m, 6H), $6.99(\mathrm{~d}, J=9.0 \mathrm{~Hz}, 1 \mathrm{H}), 6.79(\mathrm{~s}, 1 \mathrm{H}), 6.24(\mathrm{~d}, J=14.4 \mathrm{~Hz}, 1 \mathrm{H}), 3.75$ (s, 4H), $3.70(\mathrm{~s}, 3 \mathrm{H}), 3.46(\mathrm{~s}, 4 \mathrm{H}), 3.23(\mathrm{~s}, 4 \mathrm{H}), 2.76(\mathrm{dt}, J=25.0,5.9 \mathrm{~Hz}, 4 \mathrm{H}), 2.67(\mathrm{~s}, 4 \mathrm{H})$, $1.95(\mathrm{~d}, J=11.7 \mathrm{~Hz}, 2 \mathrm{H}), 1.81(\mathrm{~s}, 6 \mathrm{H}), 1.47(\mathrm{~s}, 18 \mathrm{H}) .{ }^{13} \mathrm{C} \mathrm{NMR}\left(100 \mathrm{MHz}, \mathrm{CD}_{3} \mathrm{OD}\right) \delta=$ $176.2,172.8,164.7,157.3,154.9,144.2,144.0,142.5,139.2,130.4,129.9,126.7,124.9$, $123.3,116.1,114.9,114.4,112.2,101.3,98.5,82.5,59.8,56.6,54.7,54.3,50.8,31.9,29.7$, 28.7, 28.5, 25.3, 22.0 ppm; HRMS (ESI+): $\mathrm{m} / z$ calcd for $\mathrm{C}_{44} \mathrm{H}_{59} \mathrm{~N}_{4} \mathrm{O}_{5}^{+}[\mathrm{M}]^{+\bullet} 723.4480$, found 723.4482 . 
${ }^{1} \mathrm{H}$ and ${ }^{13} \mathrm{C}$ NMR spectra of synthesized compounds
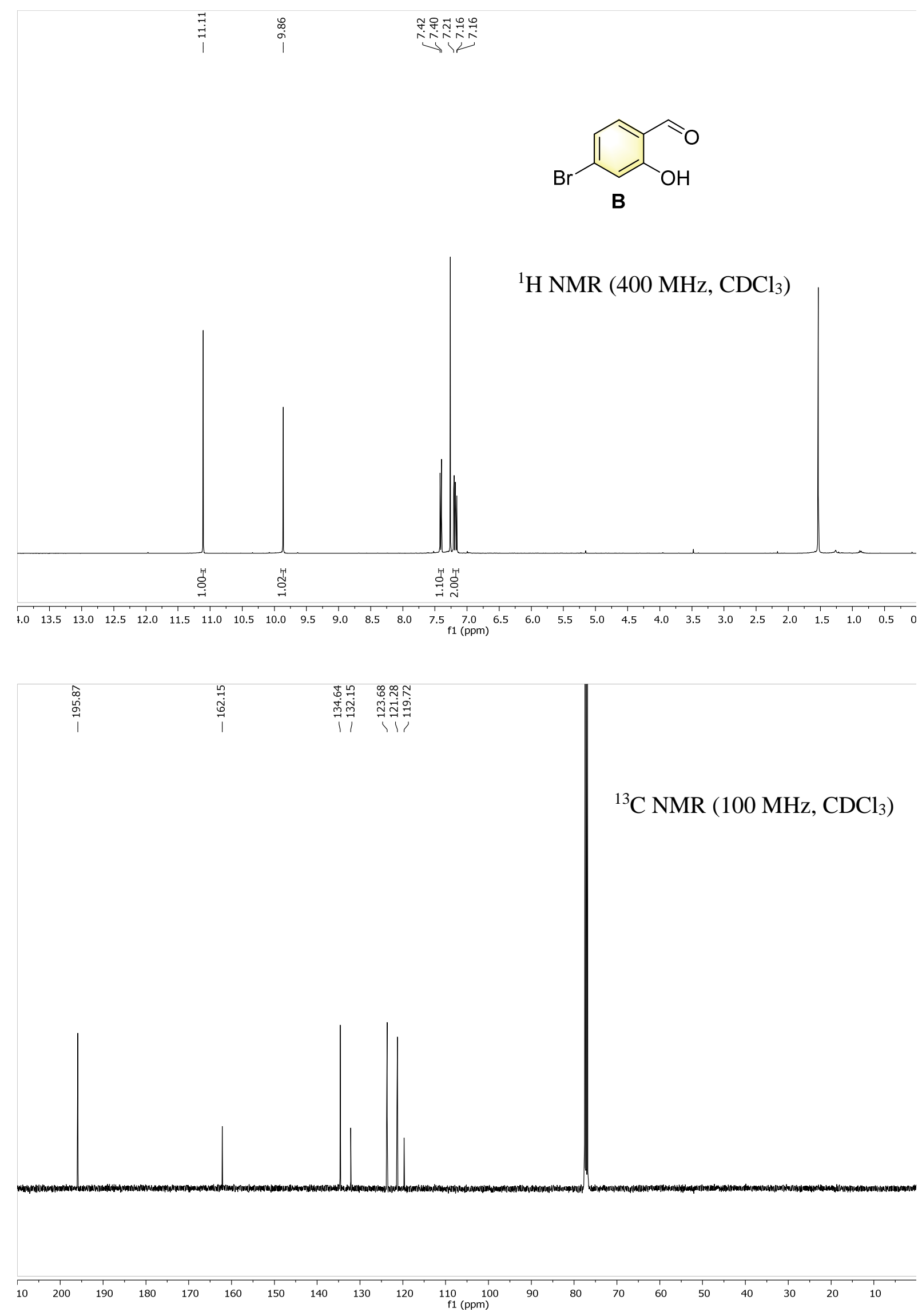

S19 

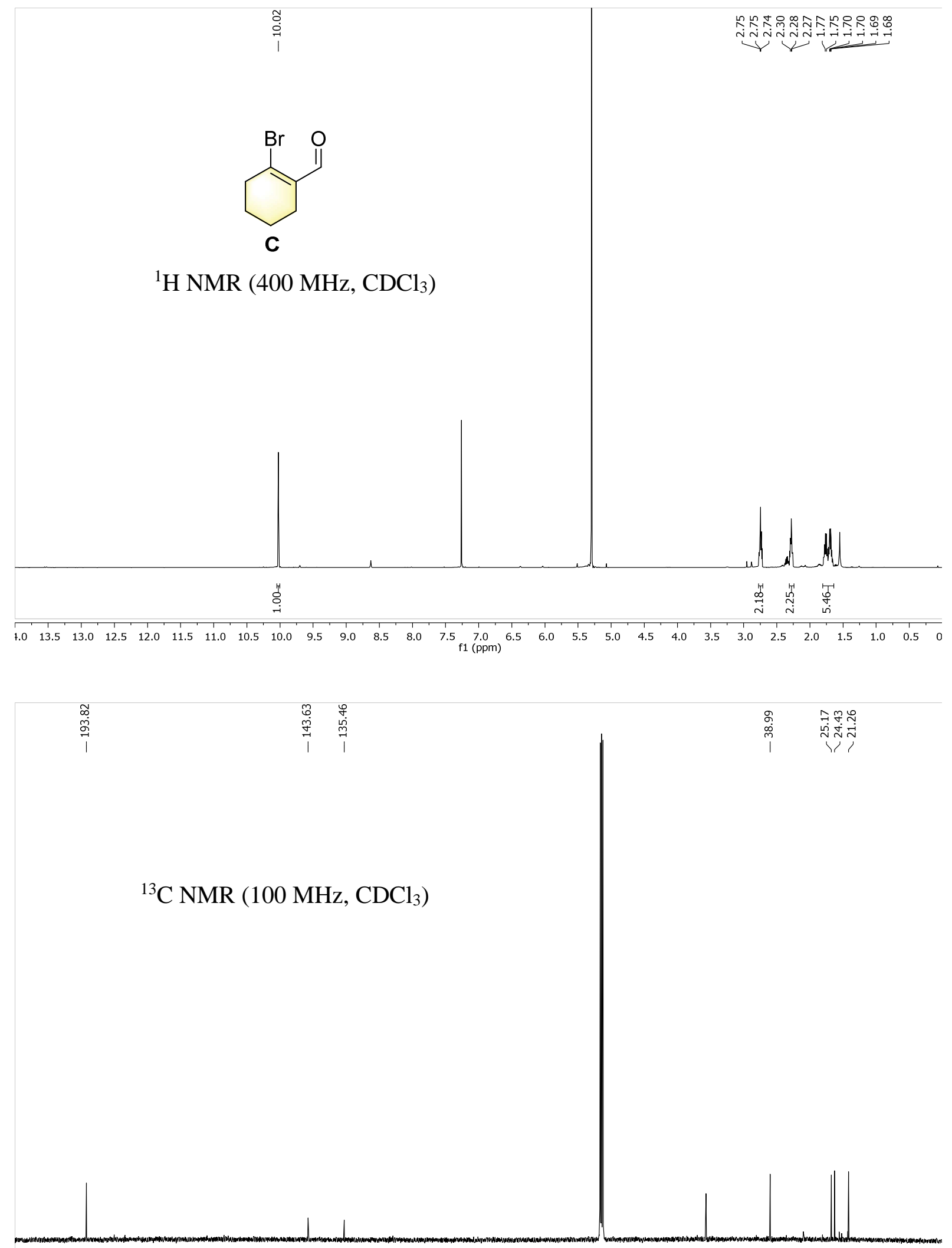

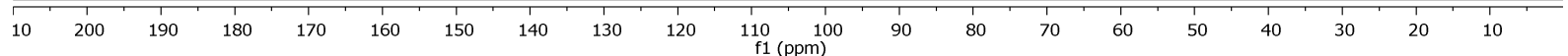



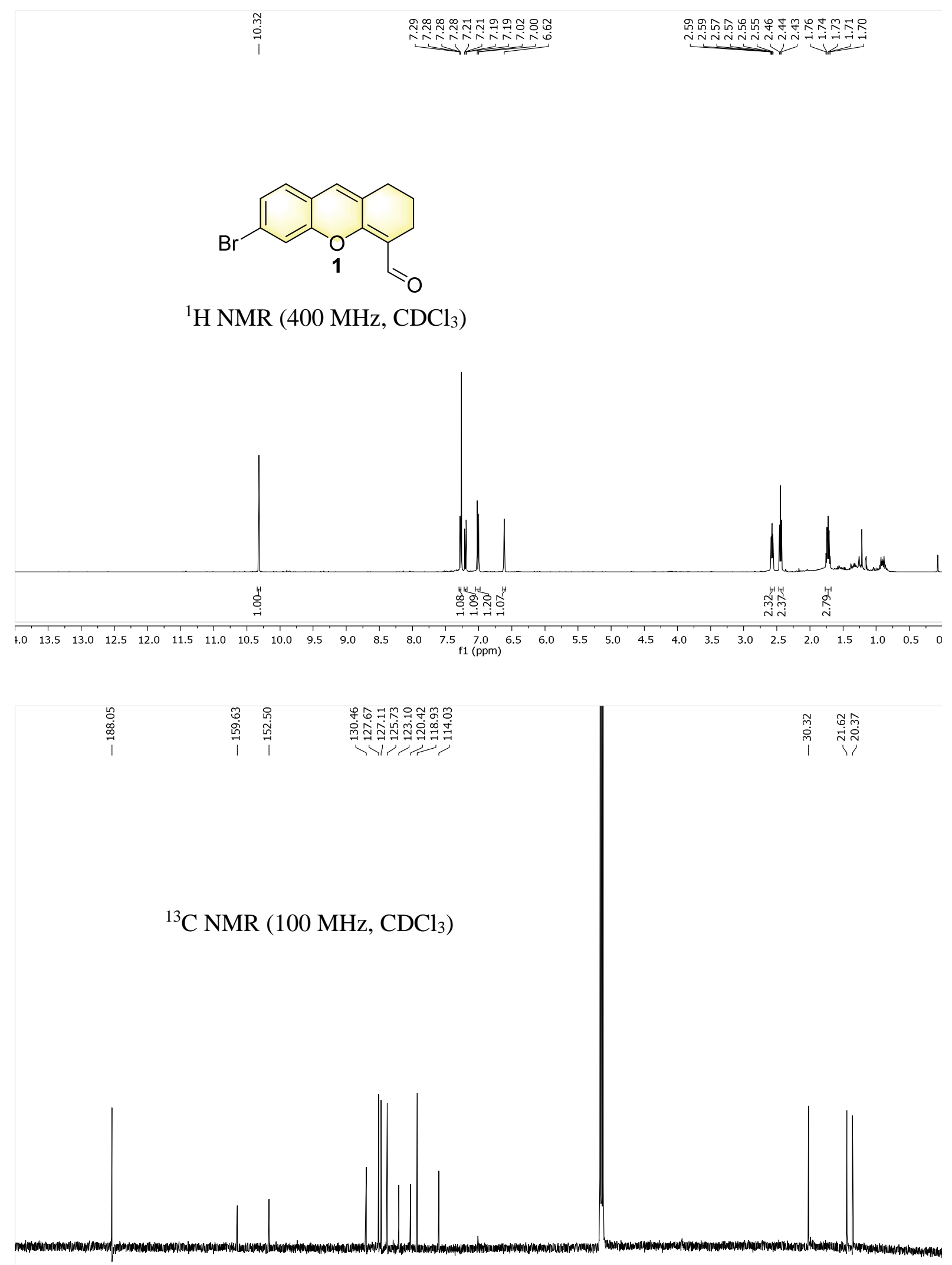

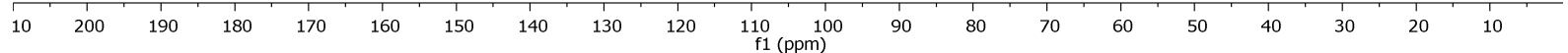



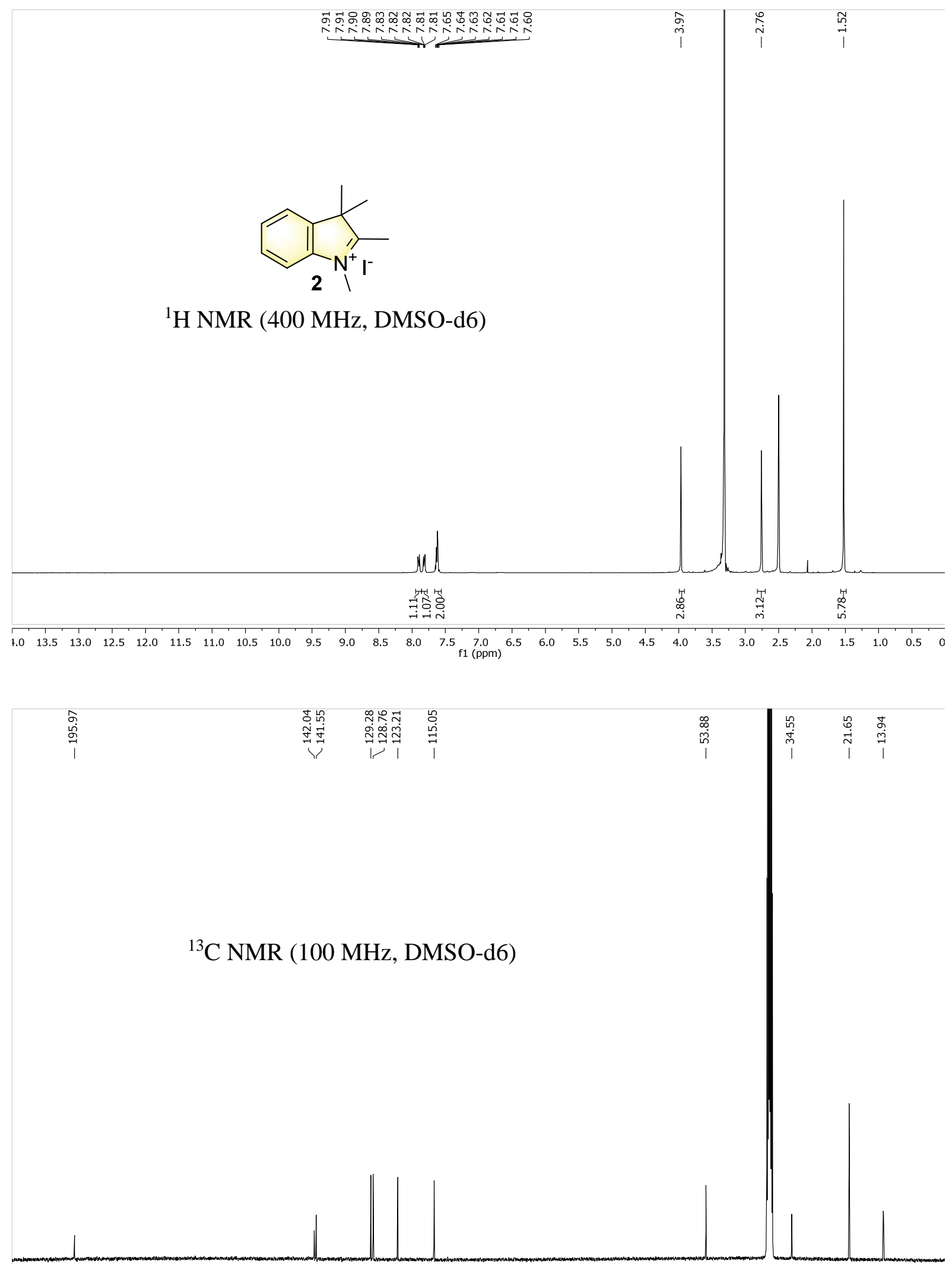

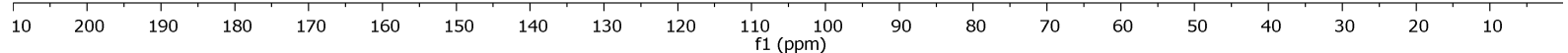




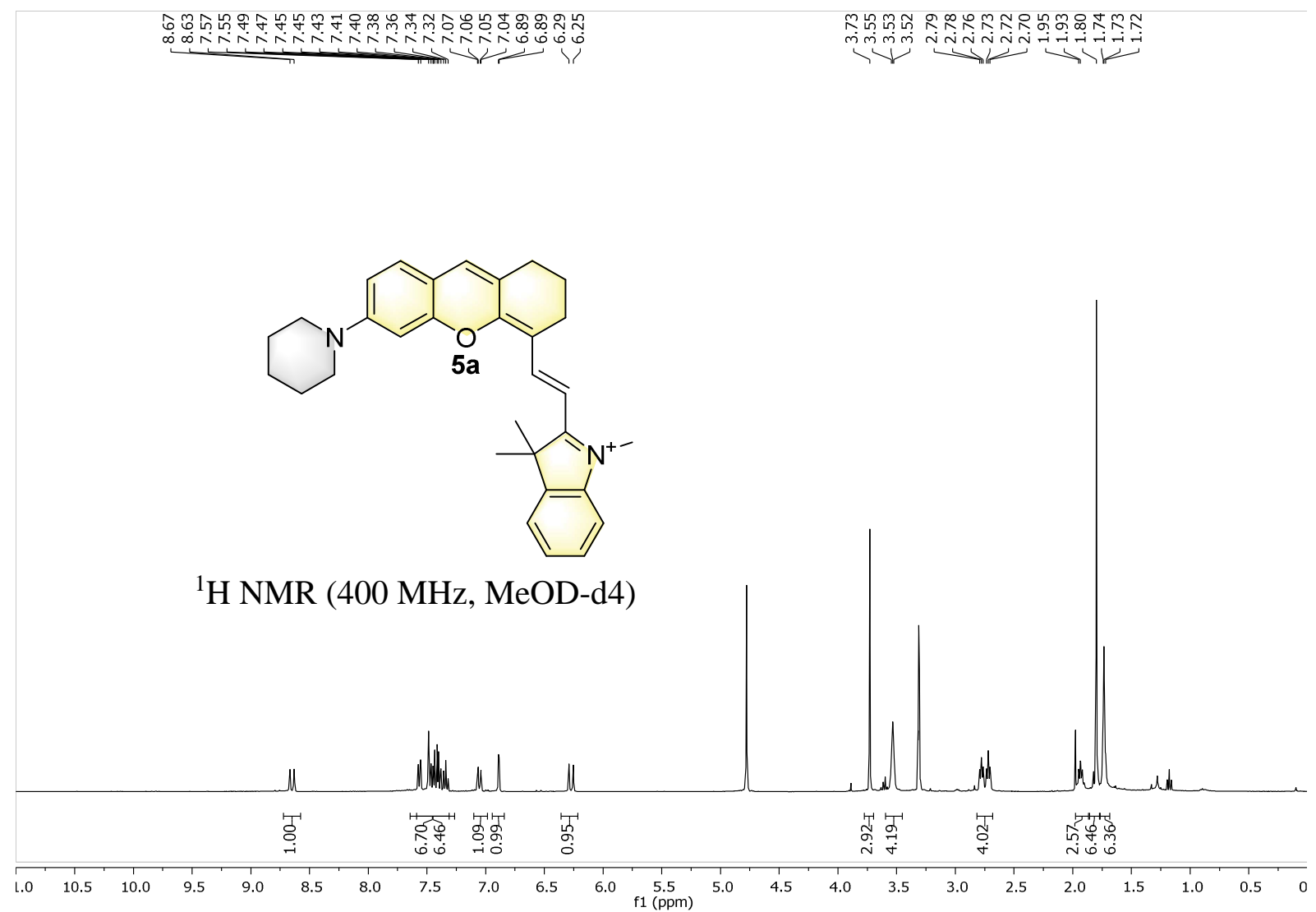

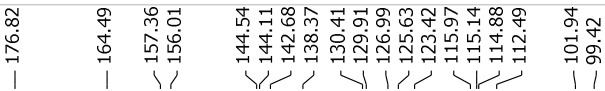

${ }^{13} \mathrm{C}$ NMR (100 MHz MeOD-d4)

$\begin{array}{lllllllllll}10 & 200 & 190 & 180 & 170 & 160 & 150 & 140 & 130 & 120 & \substack{110 \\ \mathrm{f} 1(\mathrm{ppm})}\end{array}$ 


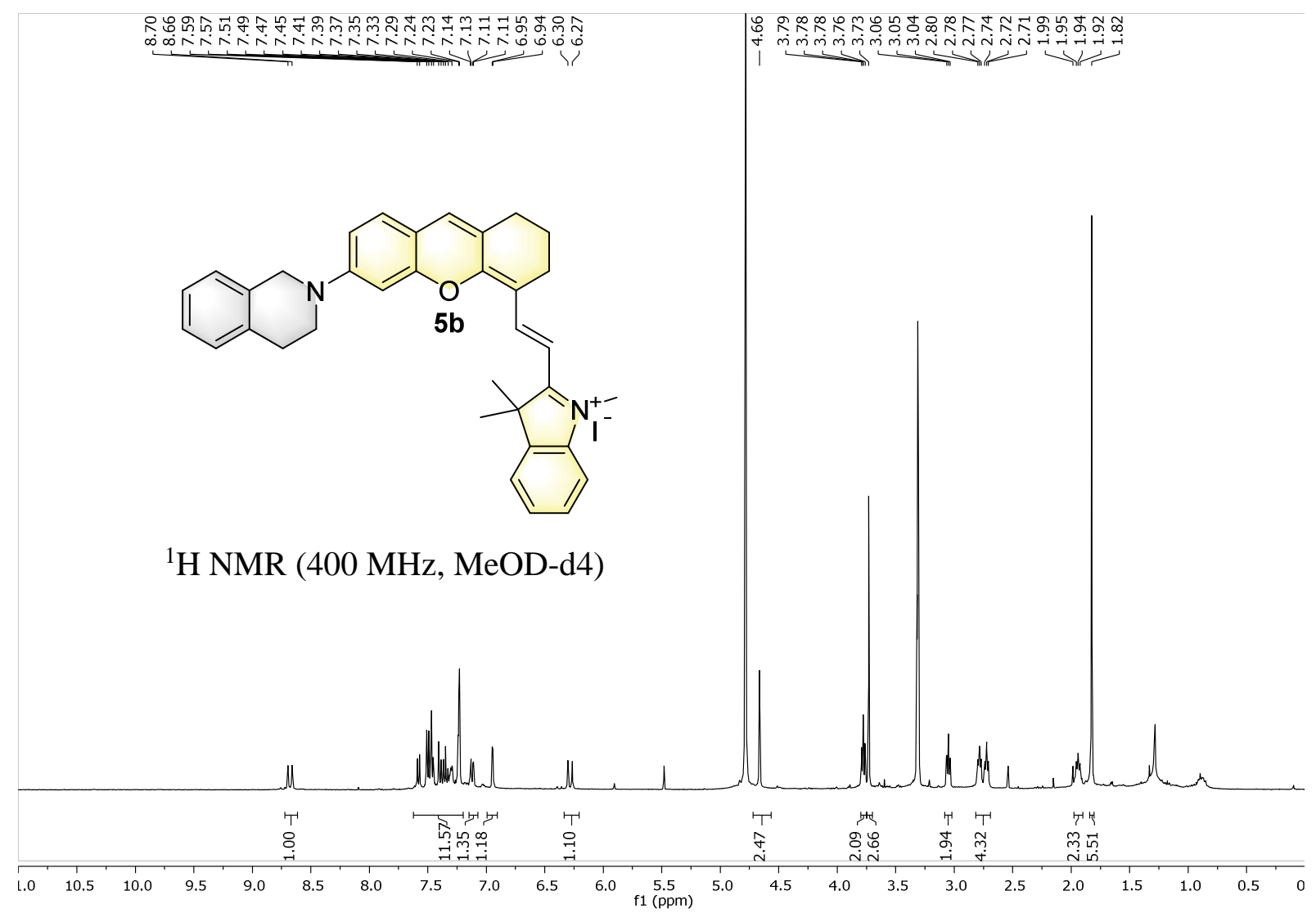

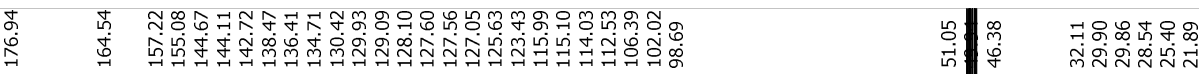

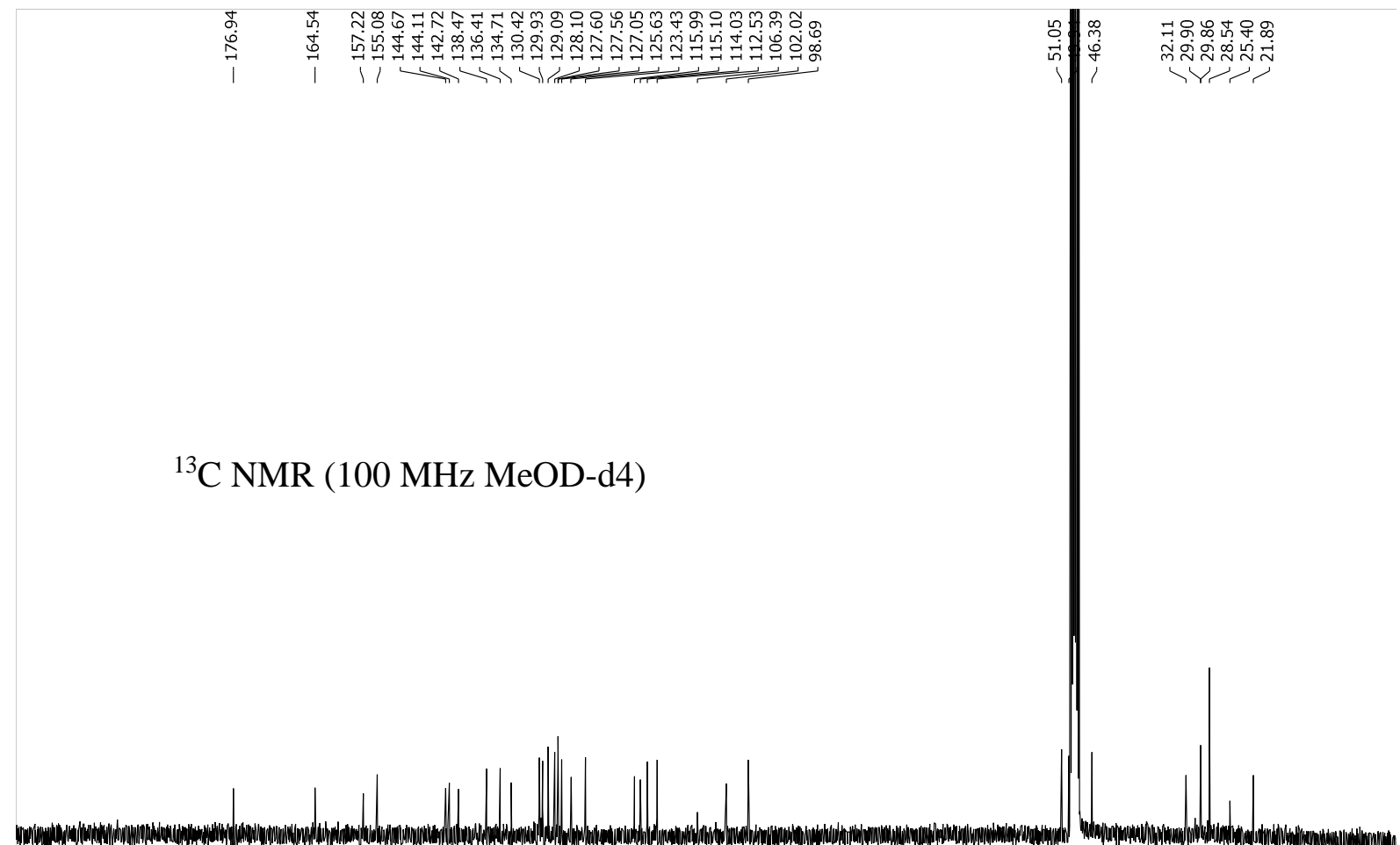

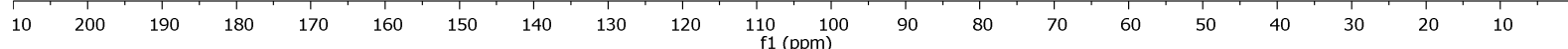




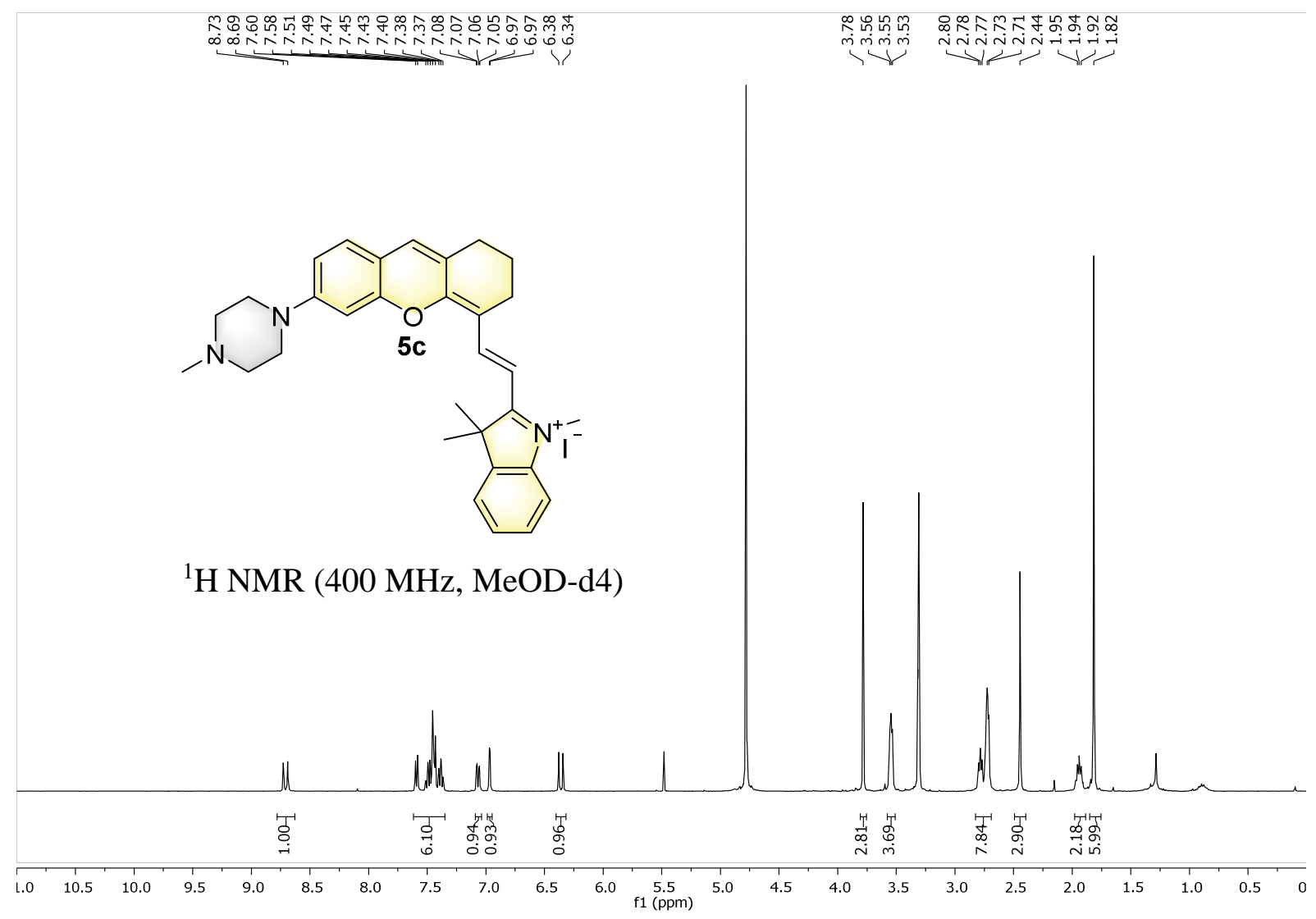

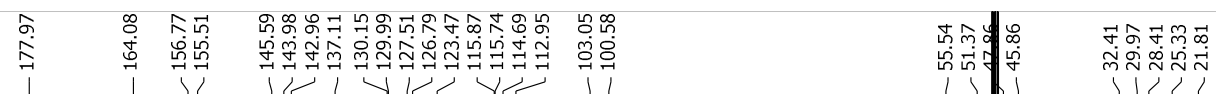

${ }^{13} \mathrm{C}$ NMR (100 MHz MeOD-d4)

$\begin{array}{lllllllllll}10 & 200 & 190 & 180 & 170 & 160 & 150 & 140 & 130 & 120 & 110 \\ \mathrm{f} 1(\mathrm{ppm})\end{array}$ 


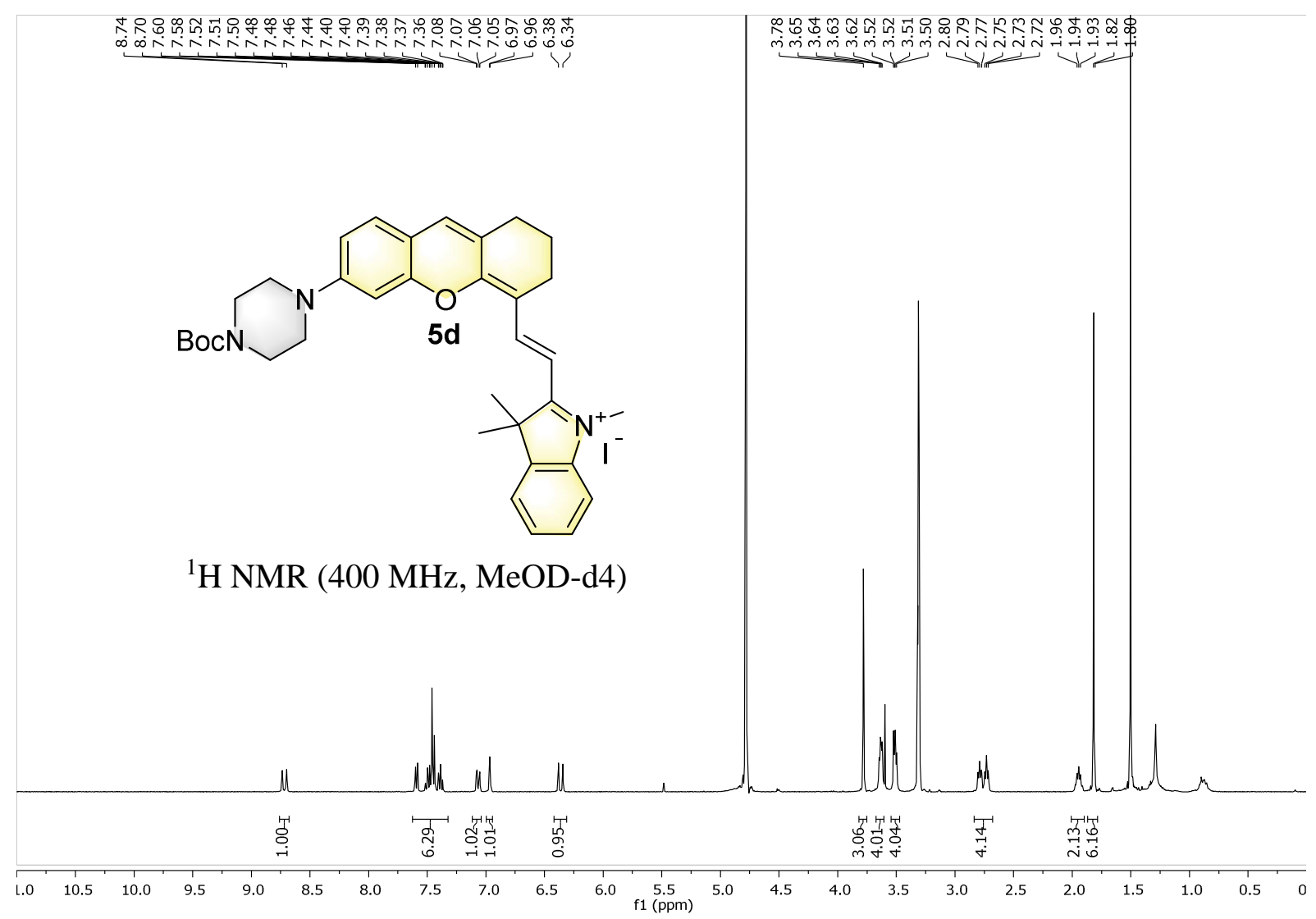

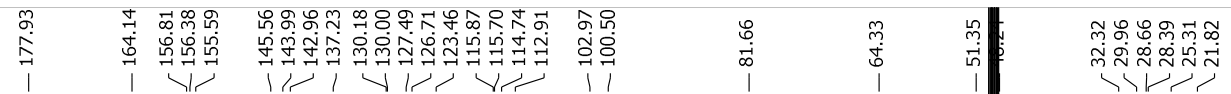

${ }^{13} \mathrm{C}$ NMR (100 MHz MeOD-d4)

$\begin{array}{lllllllllll}10 & 200 & 190 & 180 & 170 & 160 & 150 & 140 & 130 & 120 & 110 \\ \mathrm{f} 1(\mathrm{ppm})\end{array}$ 


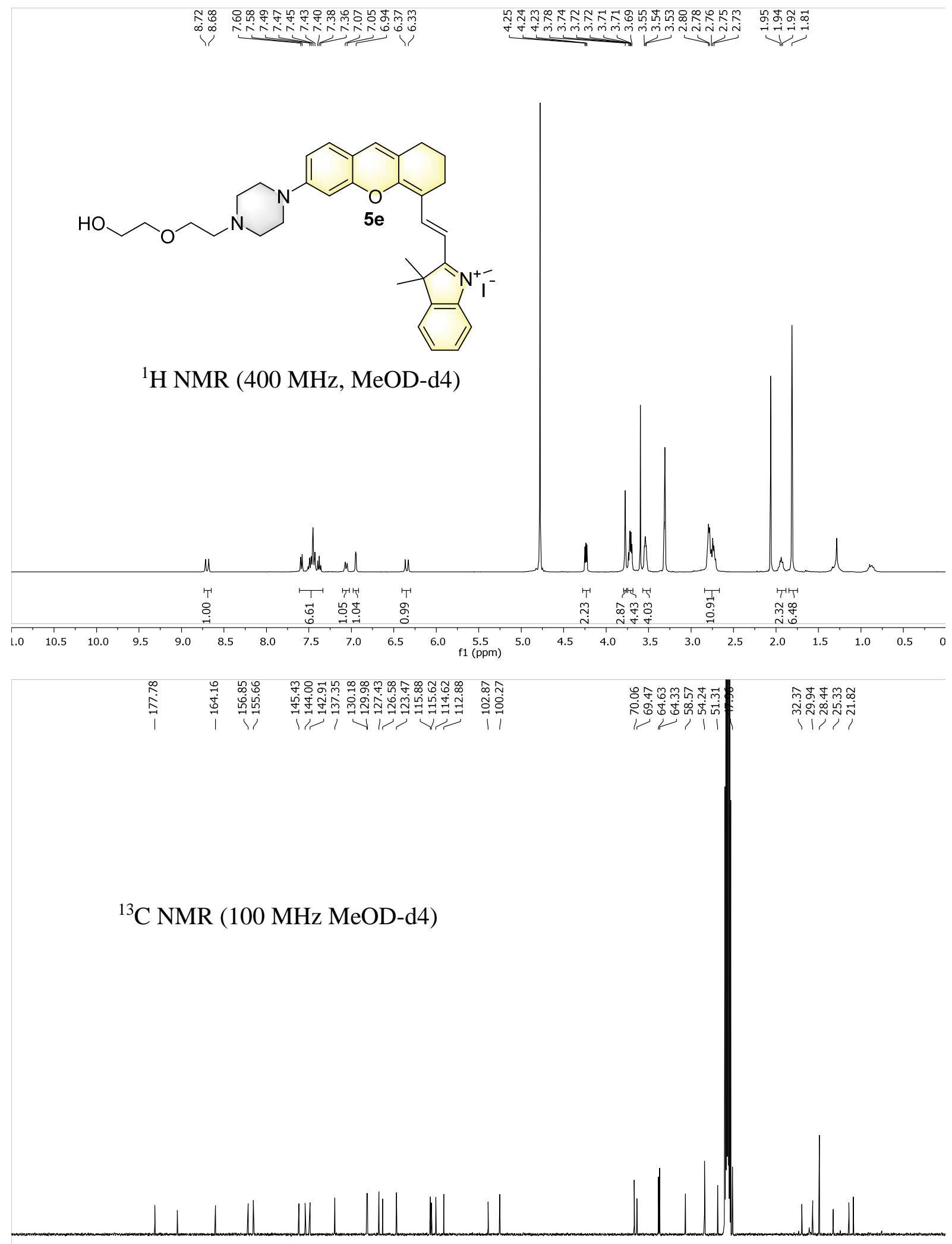

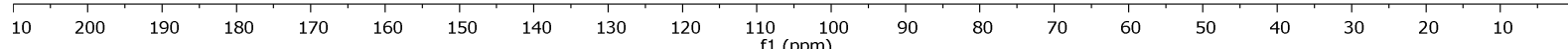



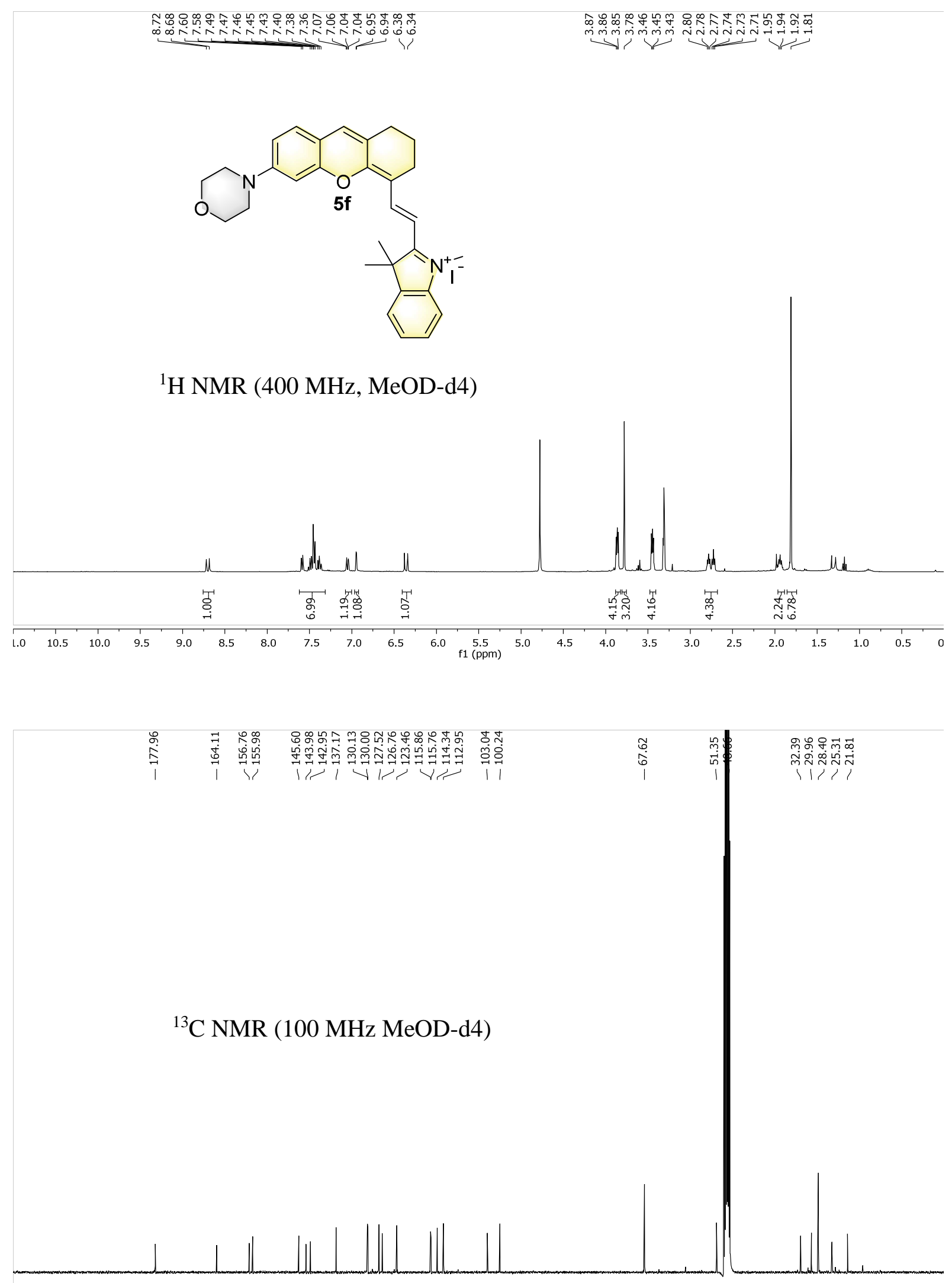

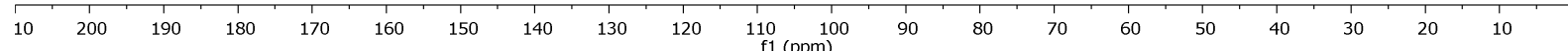




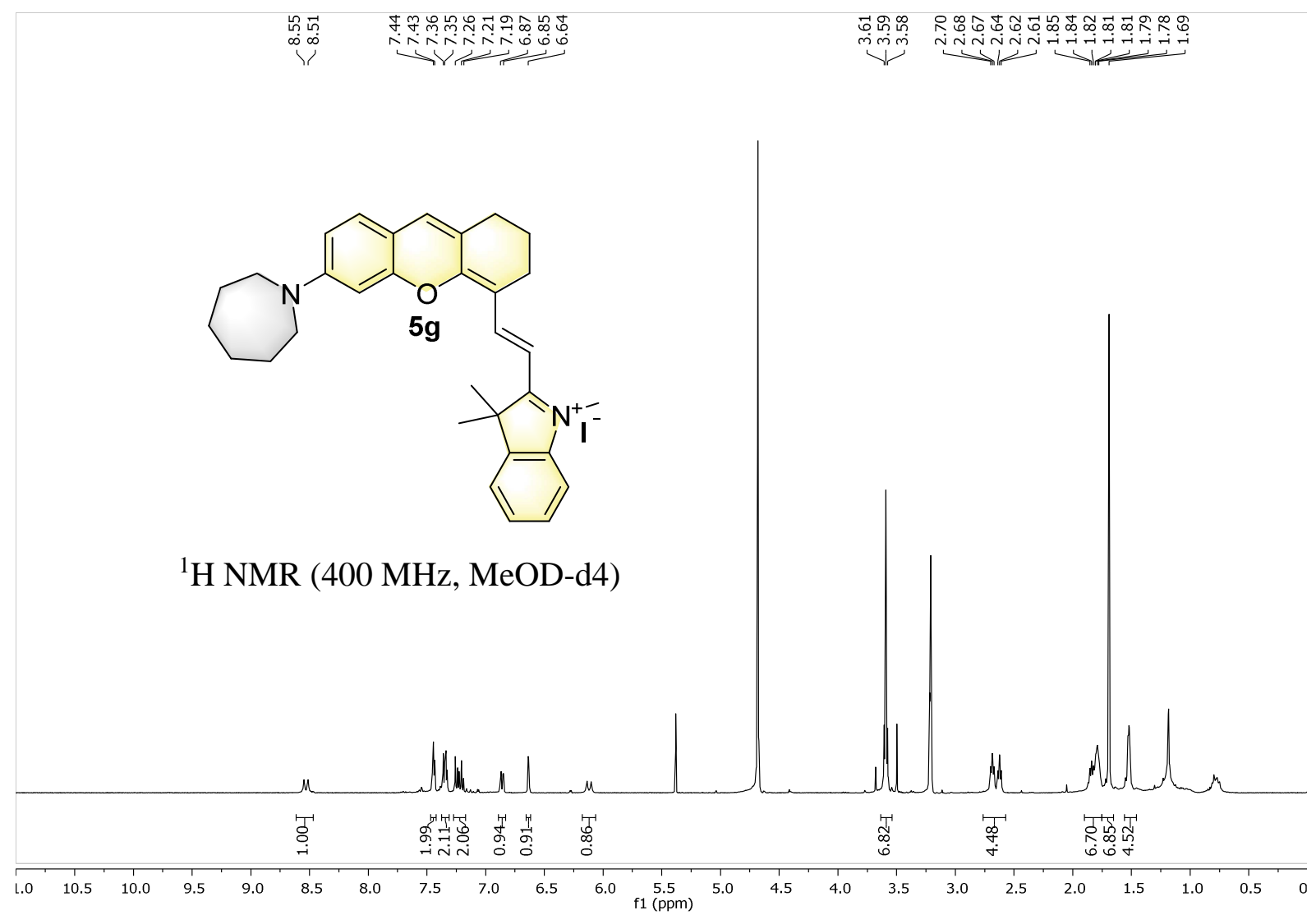

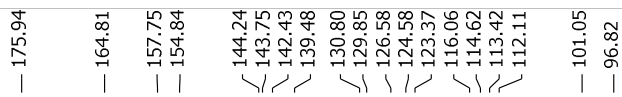

${ }^{13} \mathrm{C}$ NMR (100 MHz MeOD-d4)

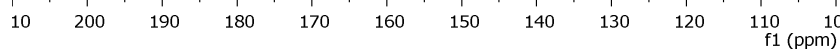




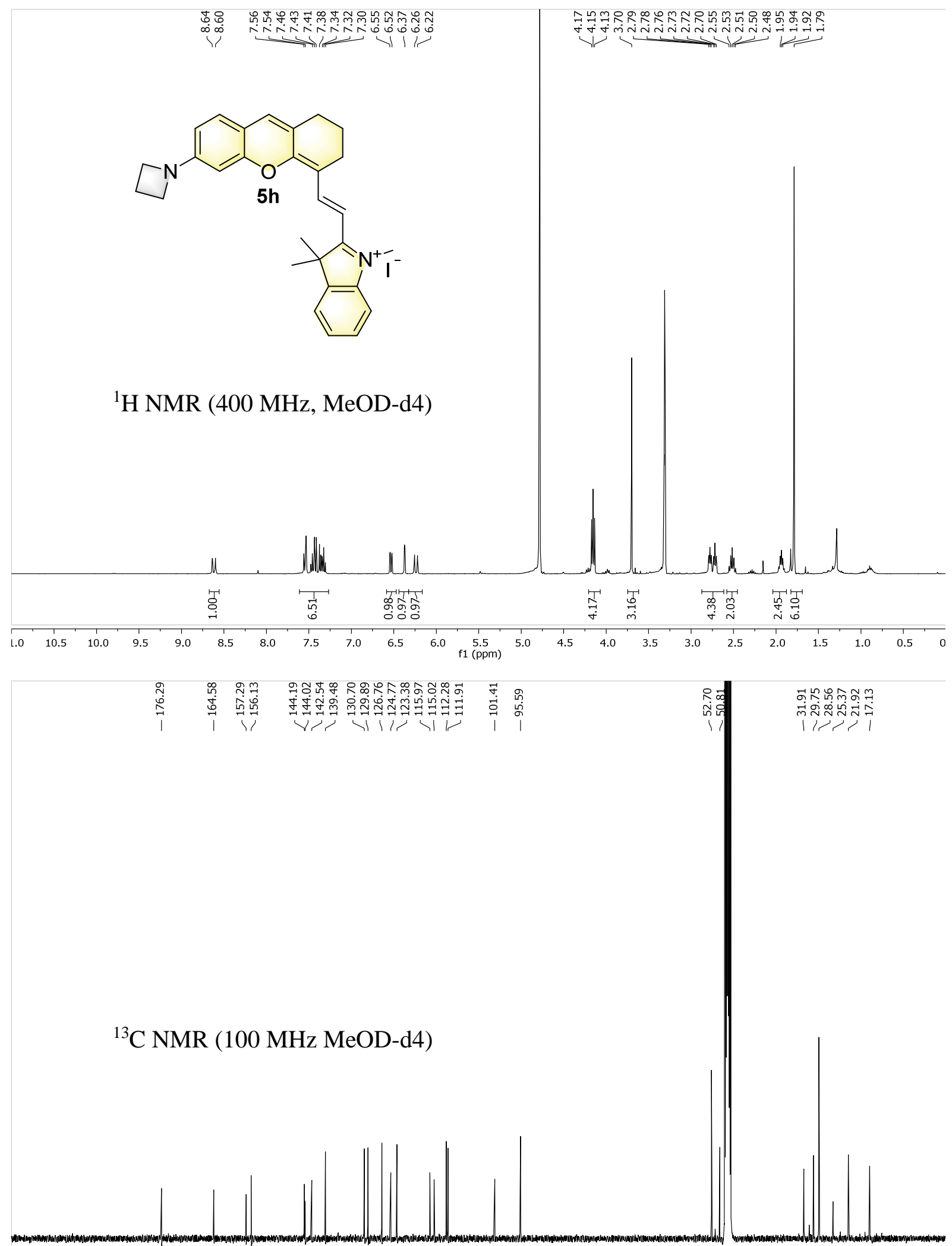

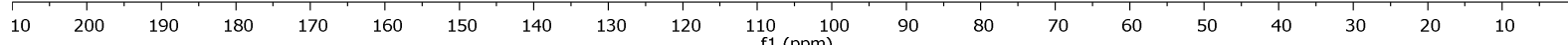




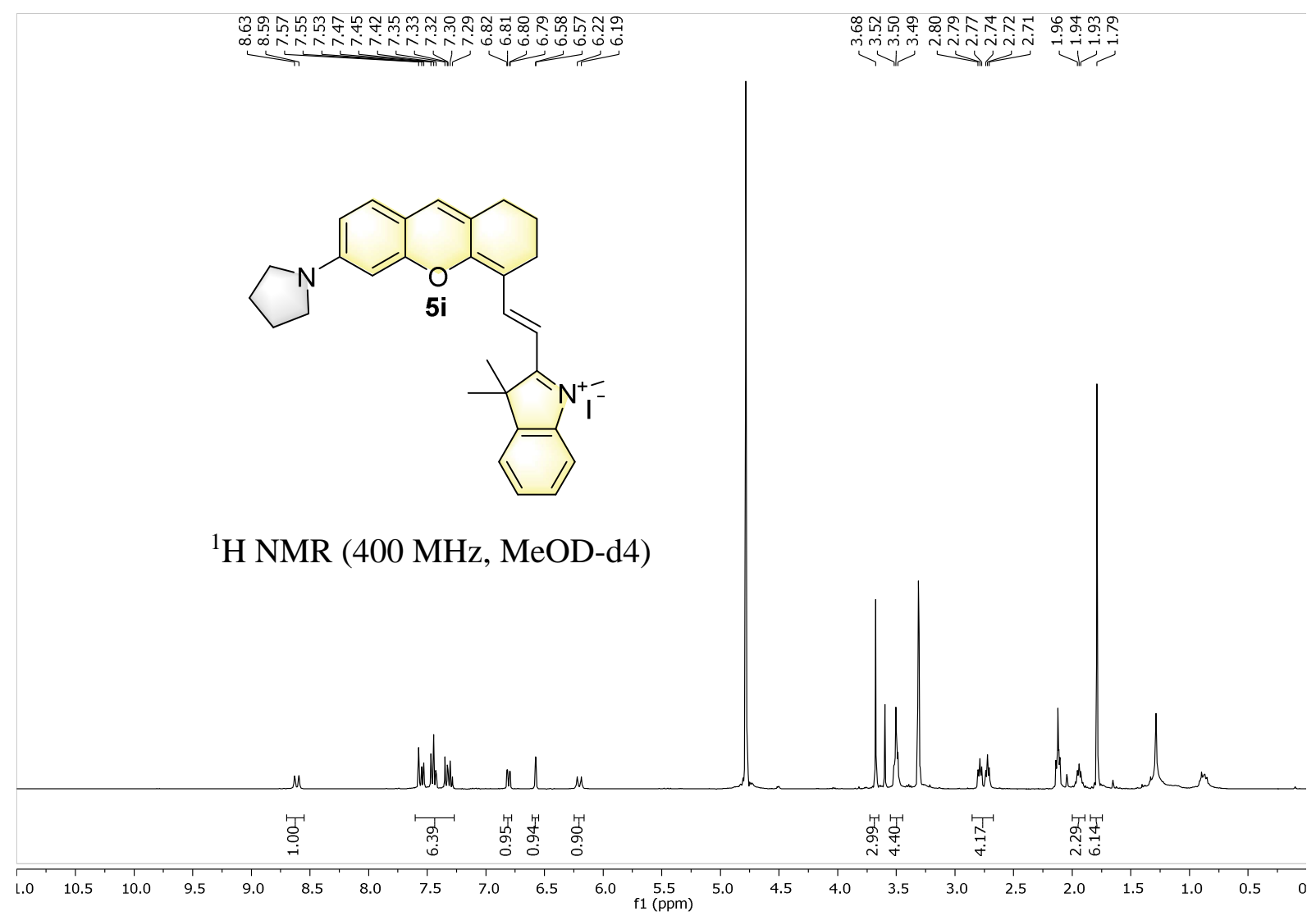

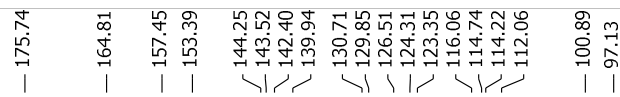

${ }^{13} \mathrm{C}$ NMR (100 MHz MeOD-d4)

$\begin{array}{lllllllllll}10 & 200 & 190 & 180 & 170 & 160 & 150 & 140 & 130 & 120 & 110 \\ \mathrm{f} 1(\mathrm{ppm})\end{array}$ 


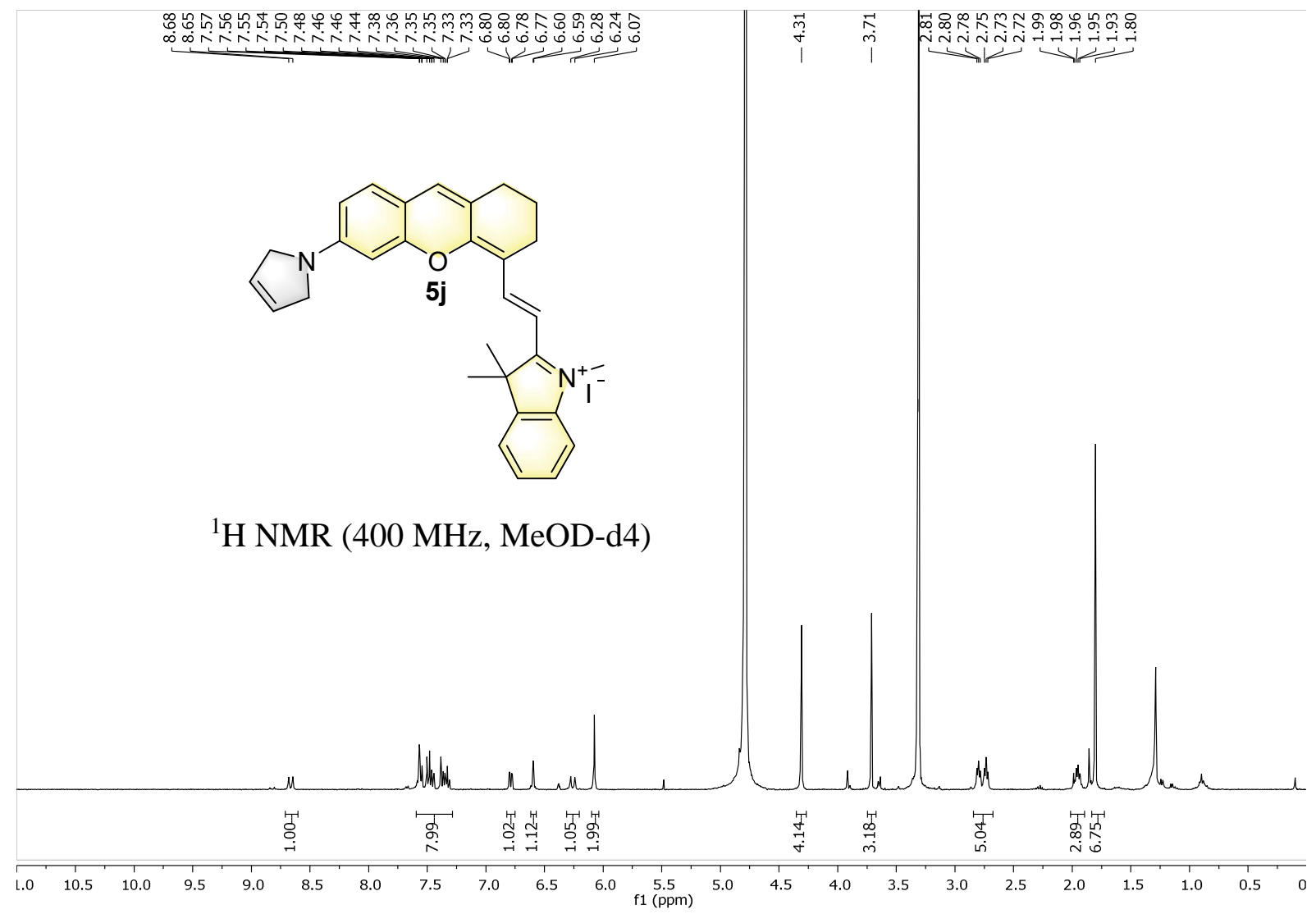

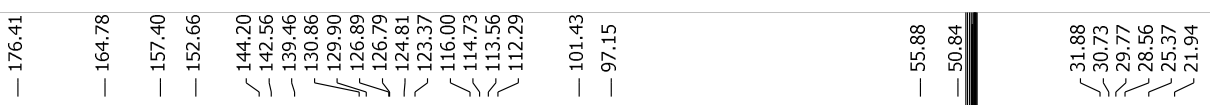

${ }^{13} \mathrm{C}$ NMR (100 MHz MeOD-d4)

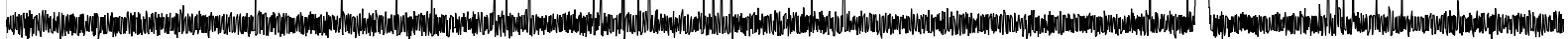

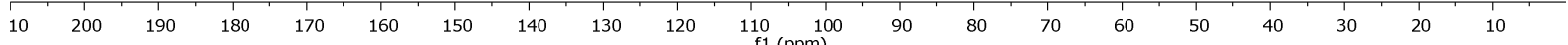




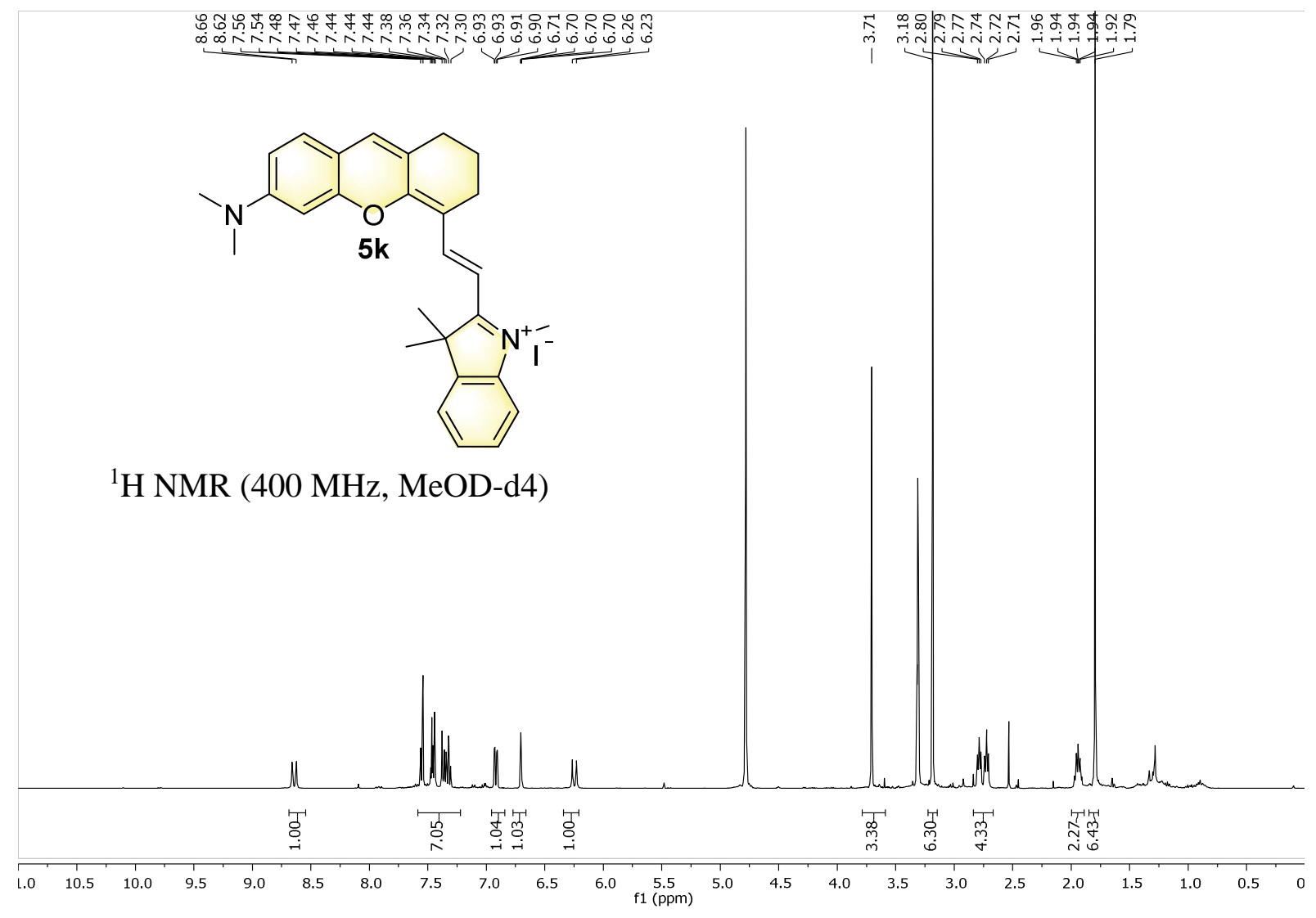

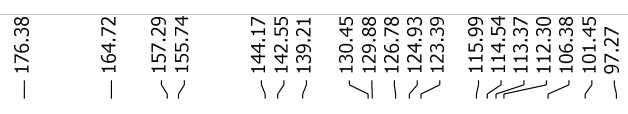

${ }^{13} \mathrm{C}$ NMR (100 MHz MeOD-d4)

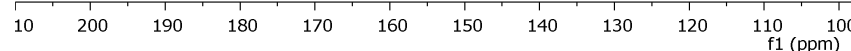




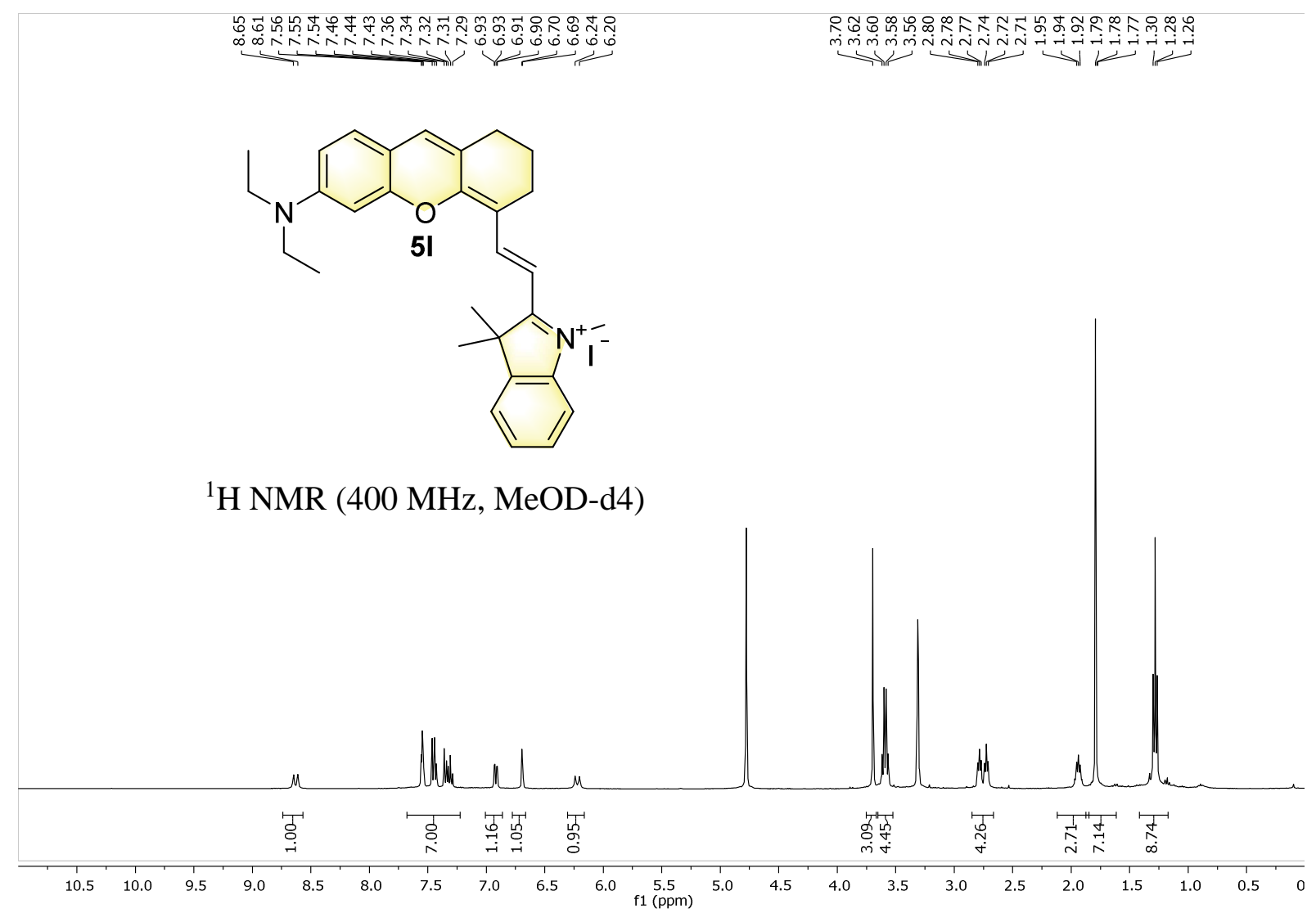

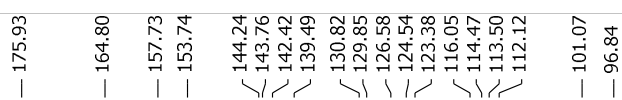

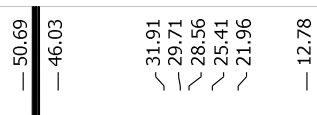

${ }^{13} \mathrm{C}$ NMR (100 MHz MeOD-d4)

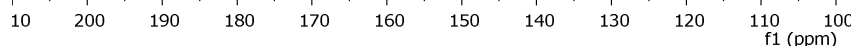
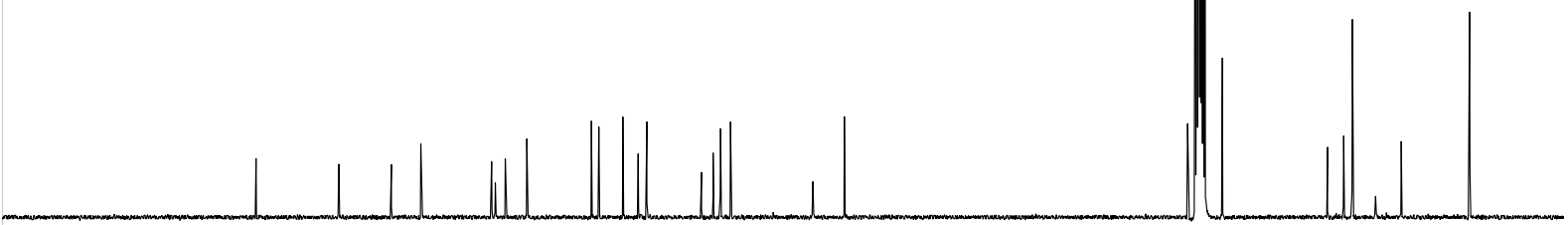

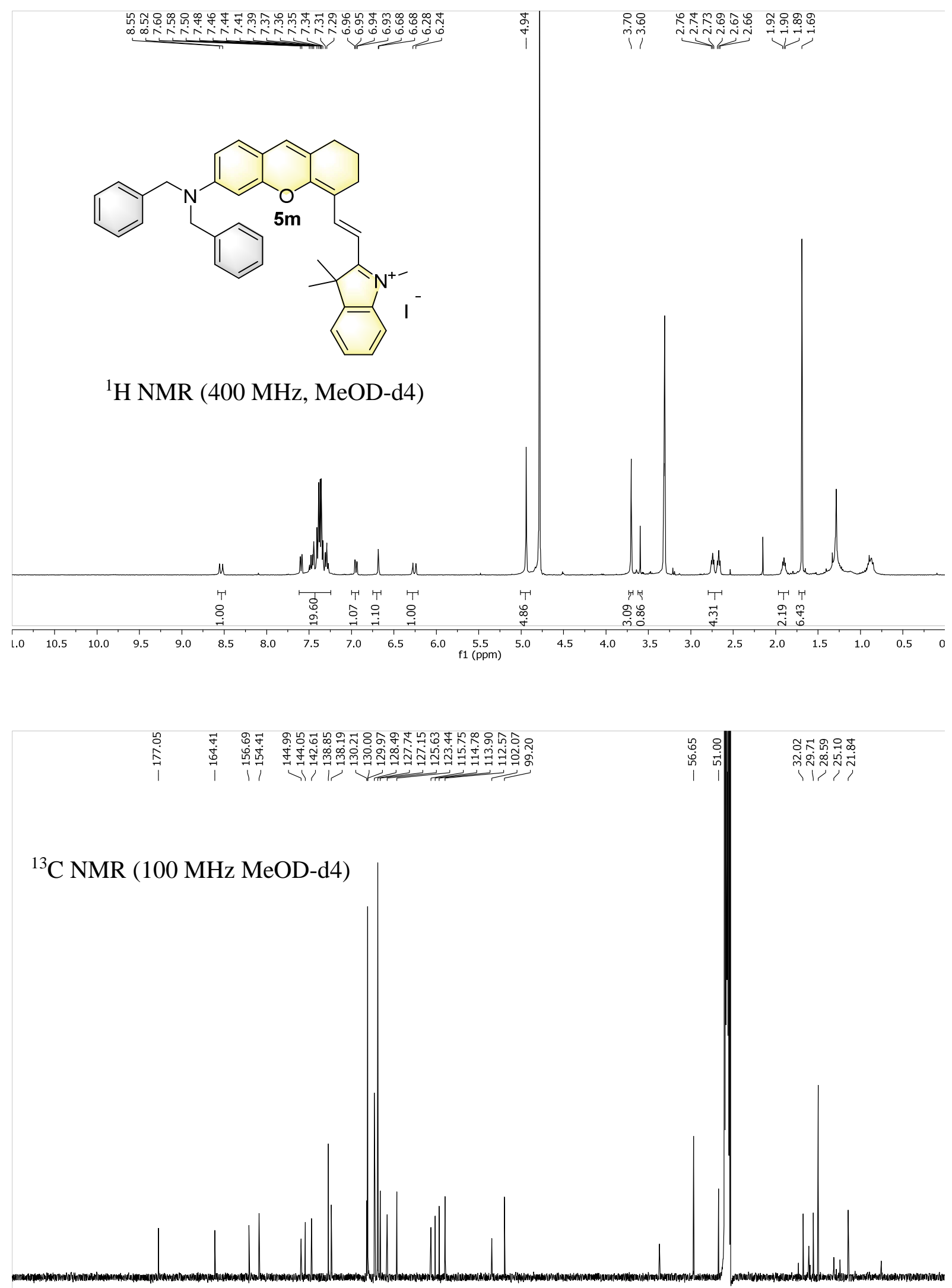

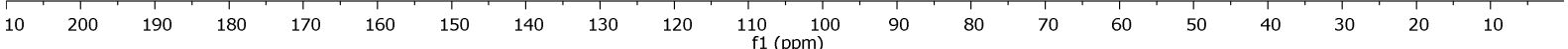




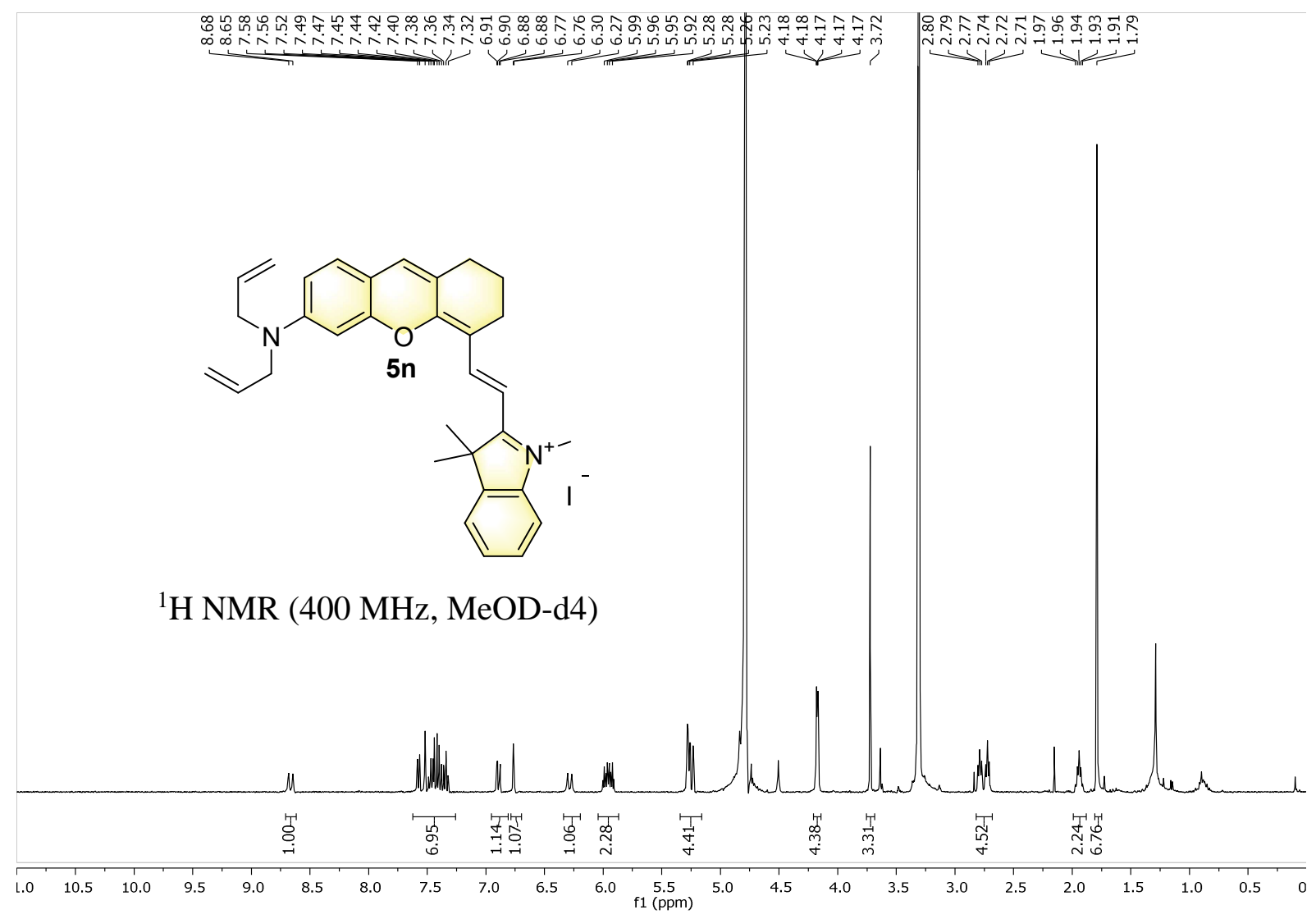

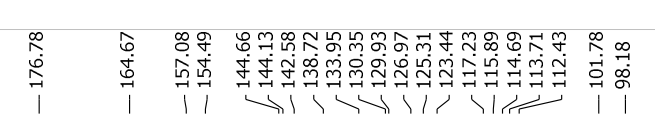

${ }^{13} \mathrm{C}$ NMR (100 MHz MeOD-d4)

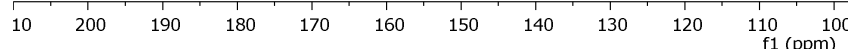




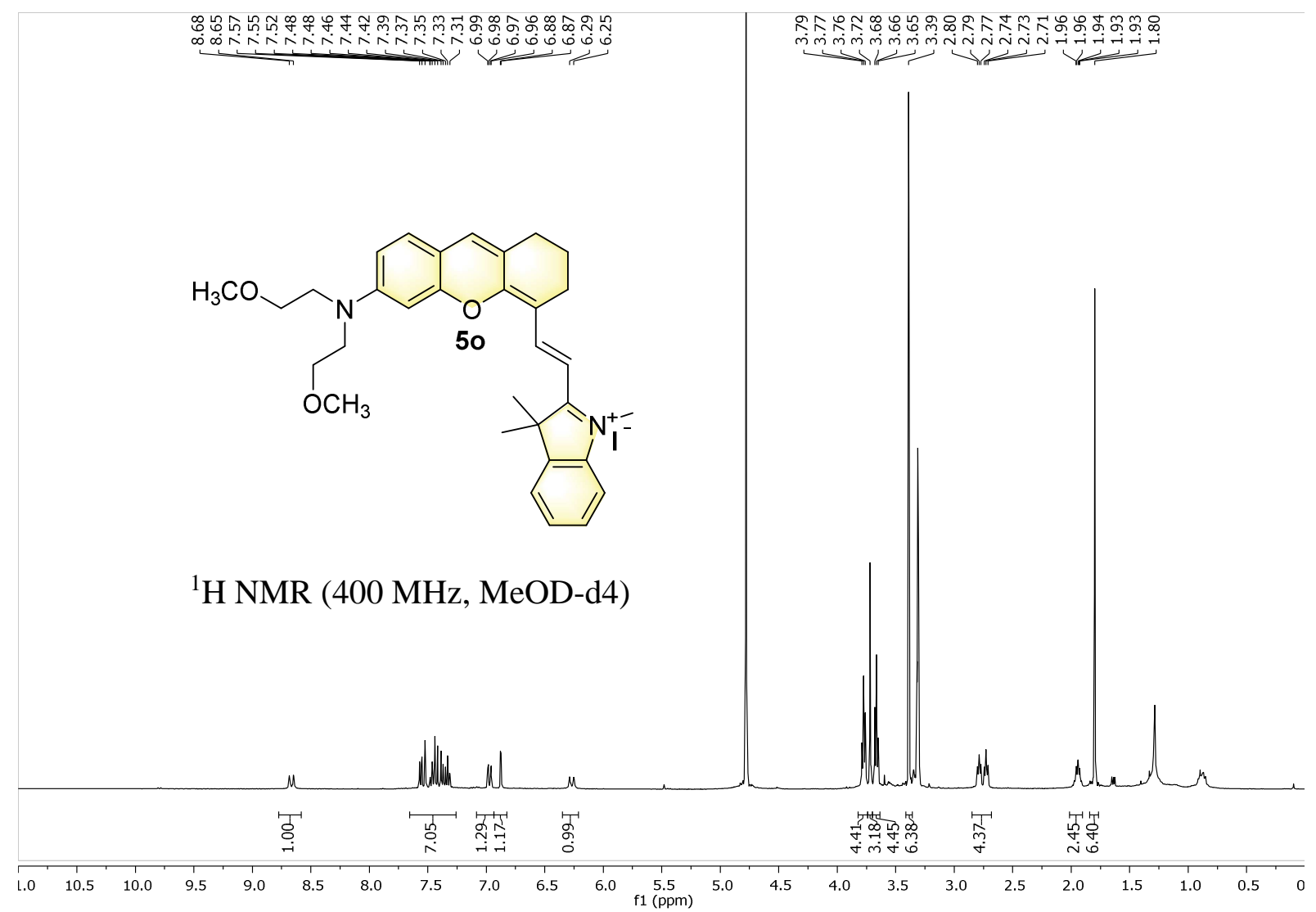

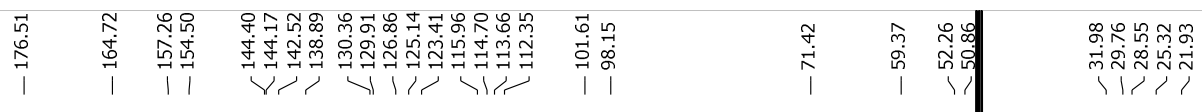

${ }^{13} \mathrm{C}$ NMR (100 MHz MeOD-d4)

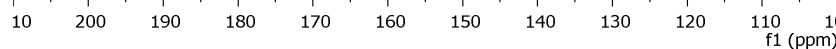




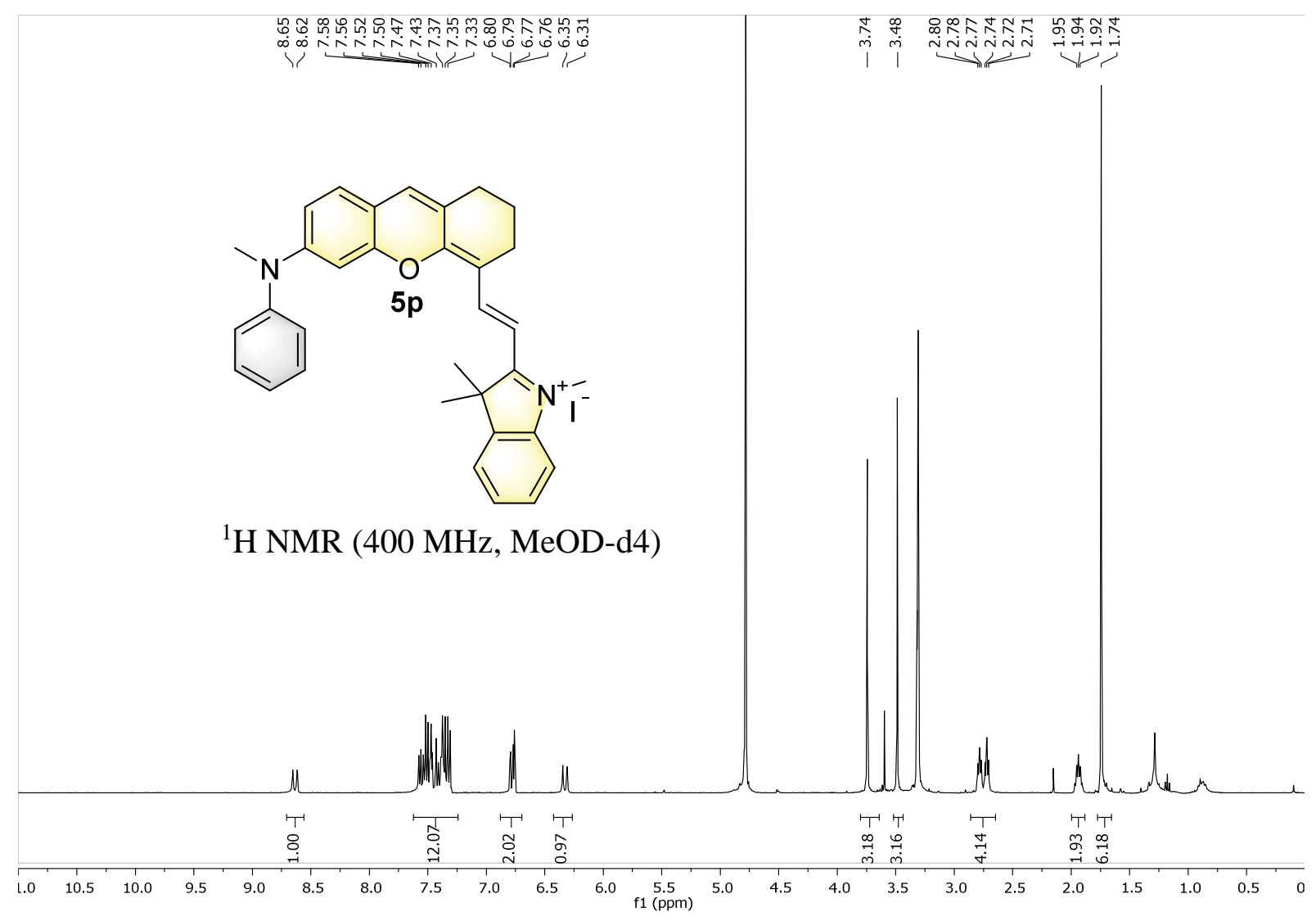

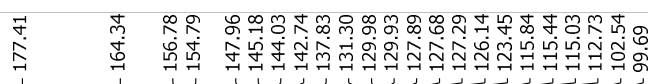

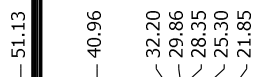

${ }^{13} \mathrm{C}$ NMR (100 MHz MeOD-d4)
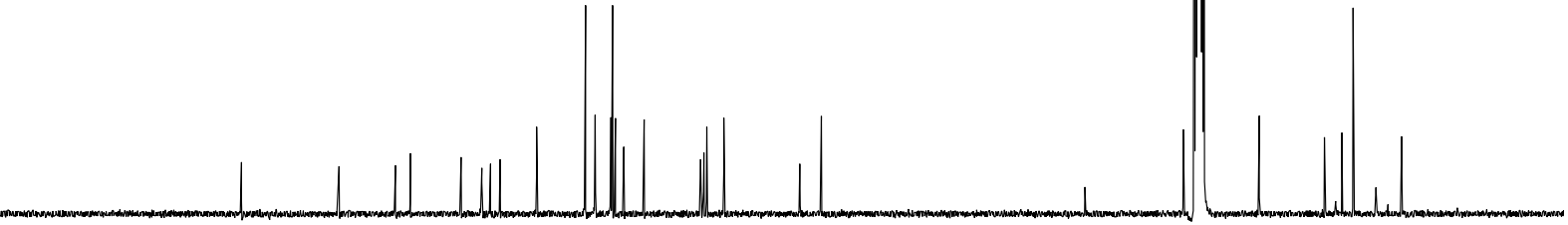

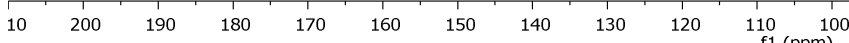



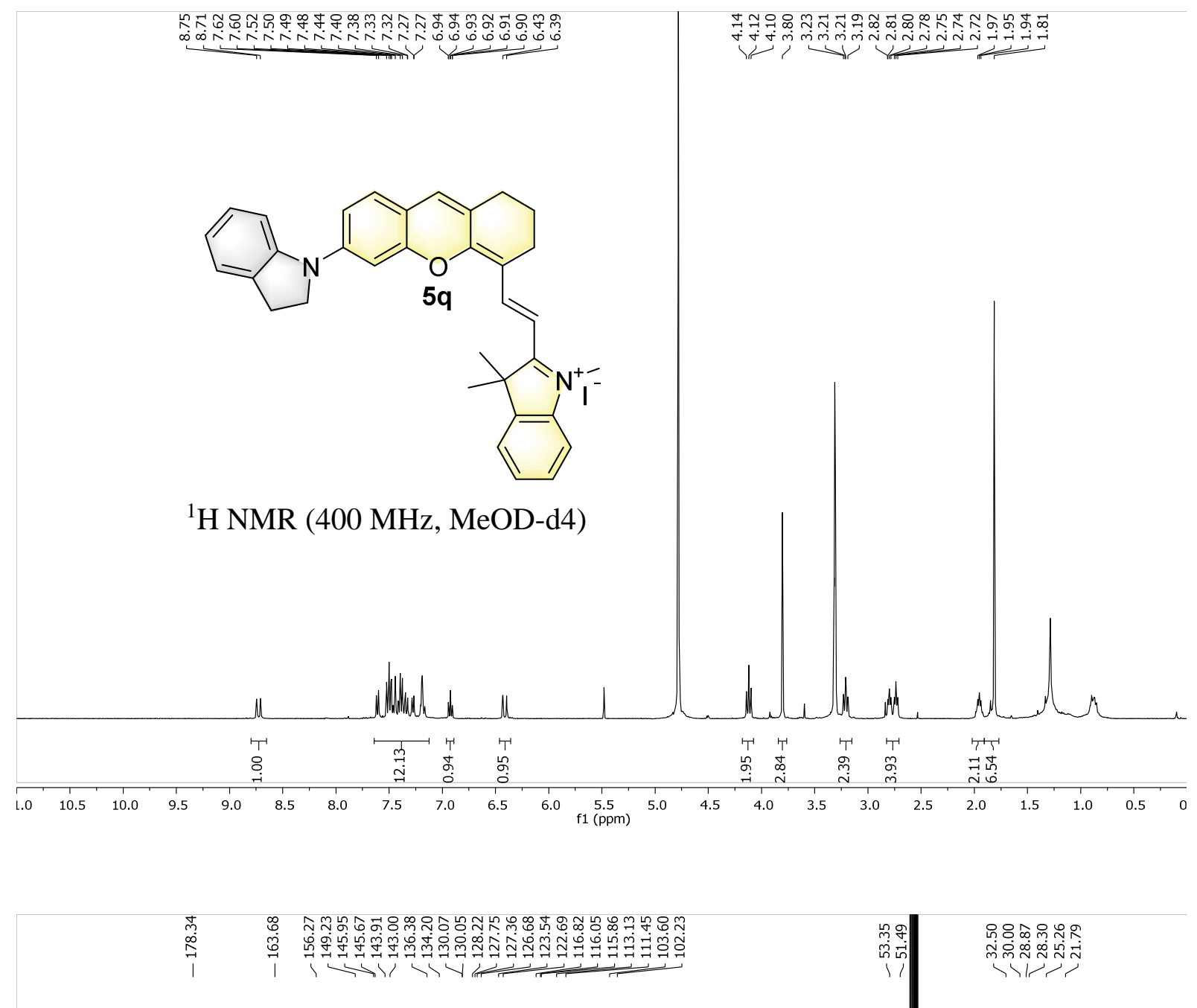

${ }^{13} \mathrm{C}$ NMR (100 MHz MeOD-d4)

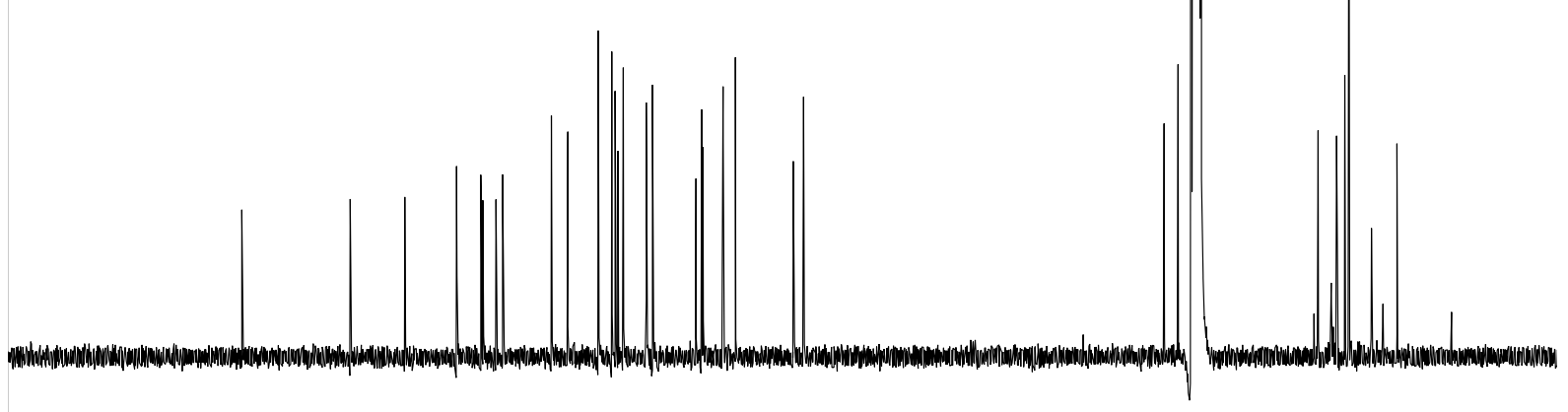

$\begin{array}{lllllllllll}10 & 200 & 190 & 180 & 170 & 160 & 150 & 140 & 130 & 120 & 110 \\ \mathrm{f} 1(\mathrm{ppm})\end{array}$ 

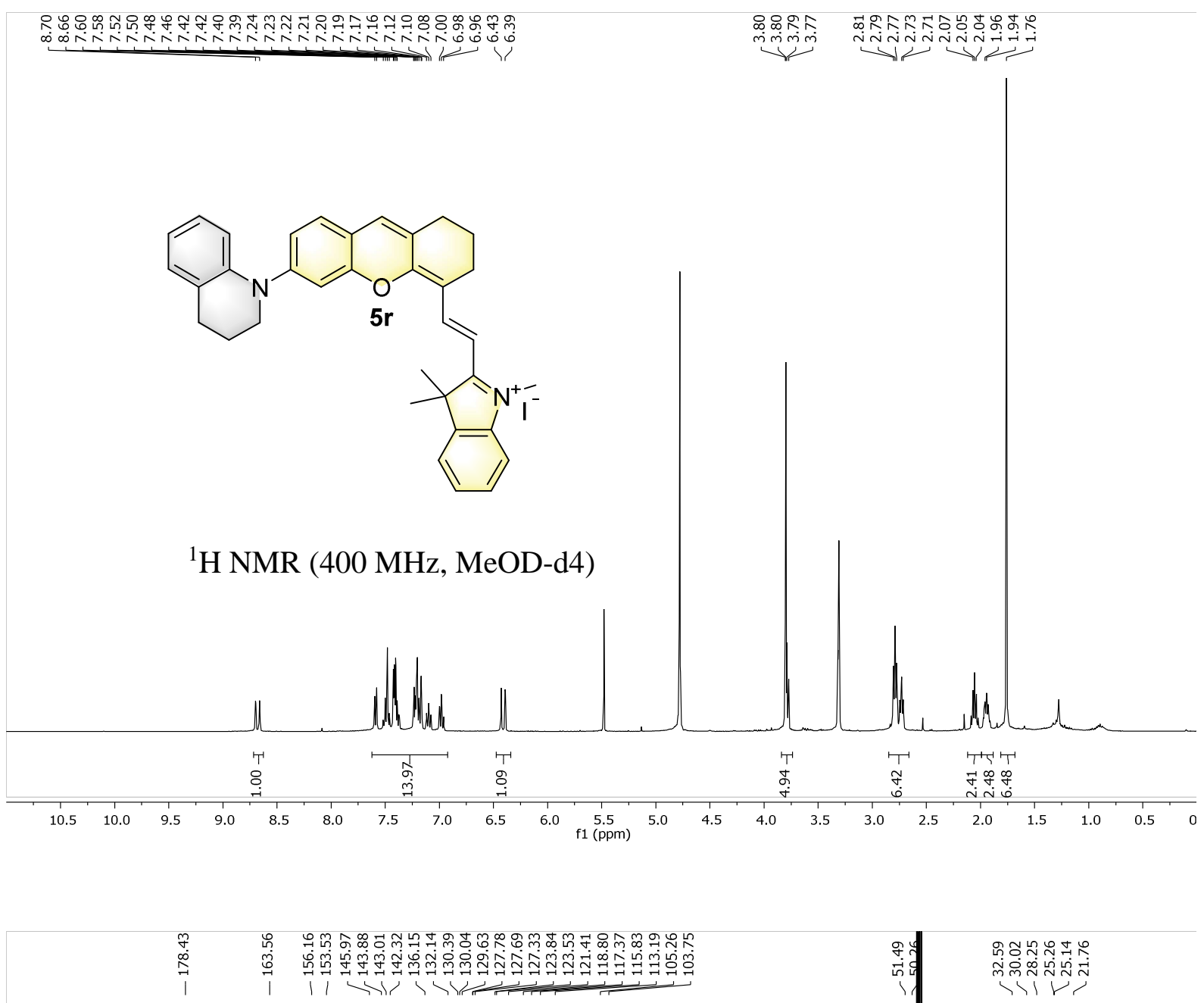

${ }^{13} \mathrm{C}$ NMR (100 MHz MeOD-d4)

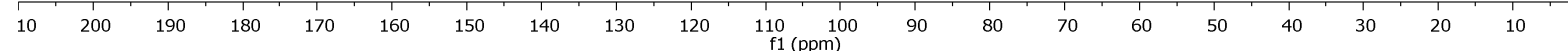




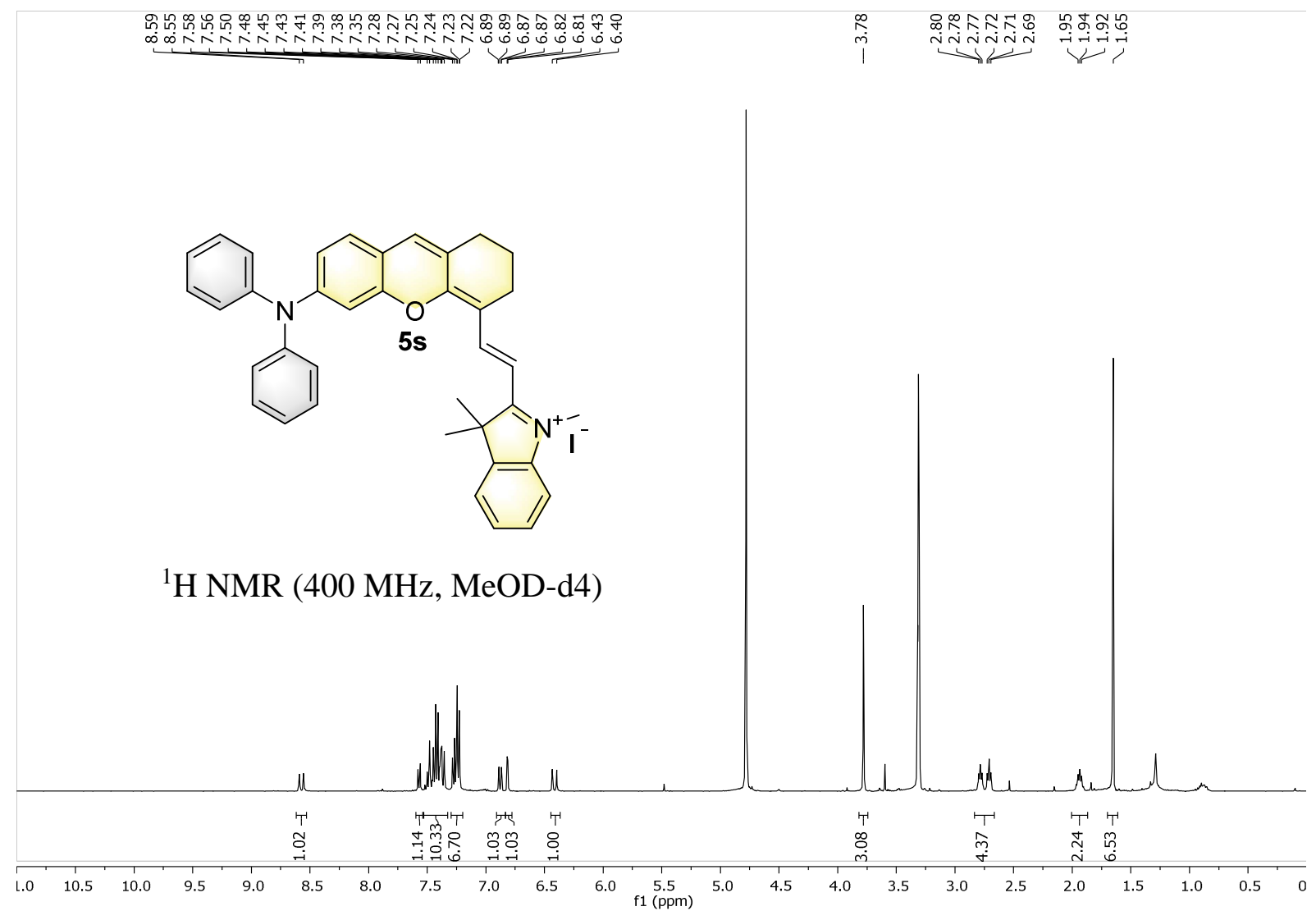

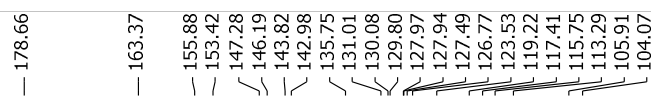

${ }^{13} \mathrm{C}$ NMR (100 MHz MeOD-d4)

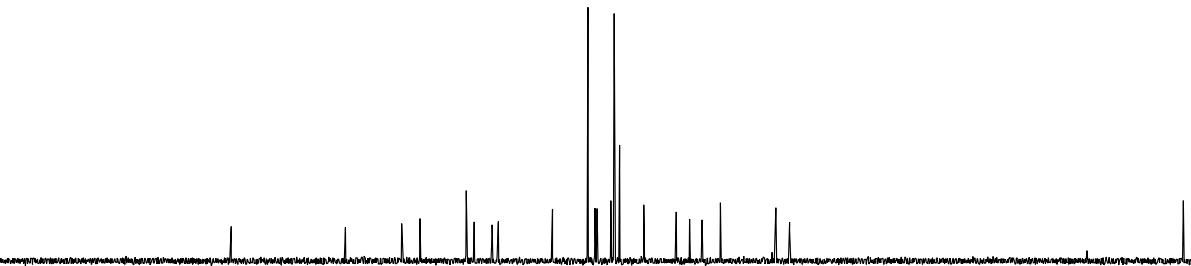

मำำ

लำํำ่

11111

$\begin{array}{llllllllllll}10 & 200 & 190 & 180 & 170 & 160 & 150 & 140 & 130 & 120 & 1100 & 100 \\ \text { f1 (ppm) }\end{array}$

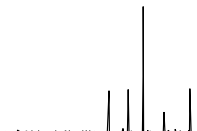



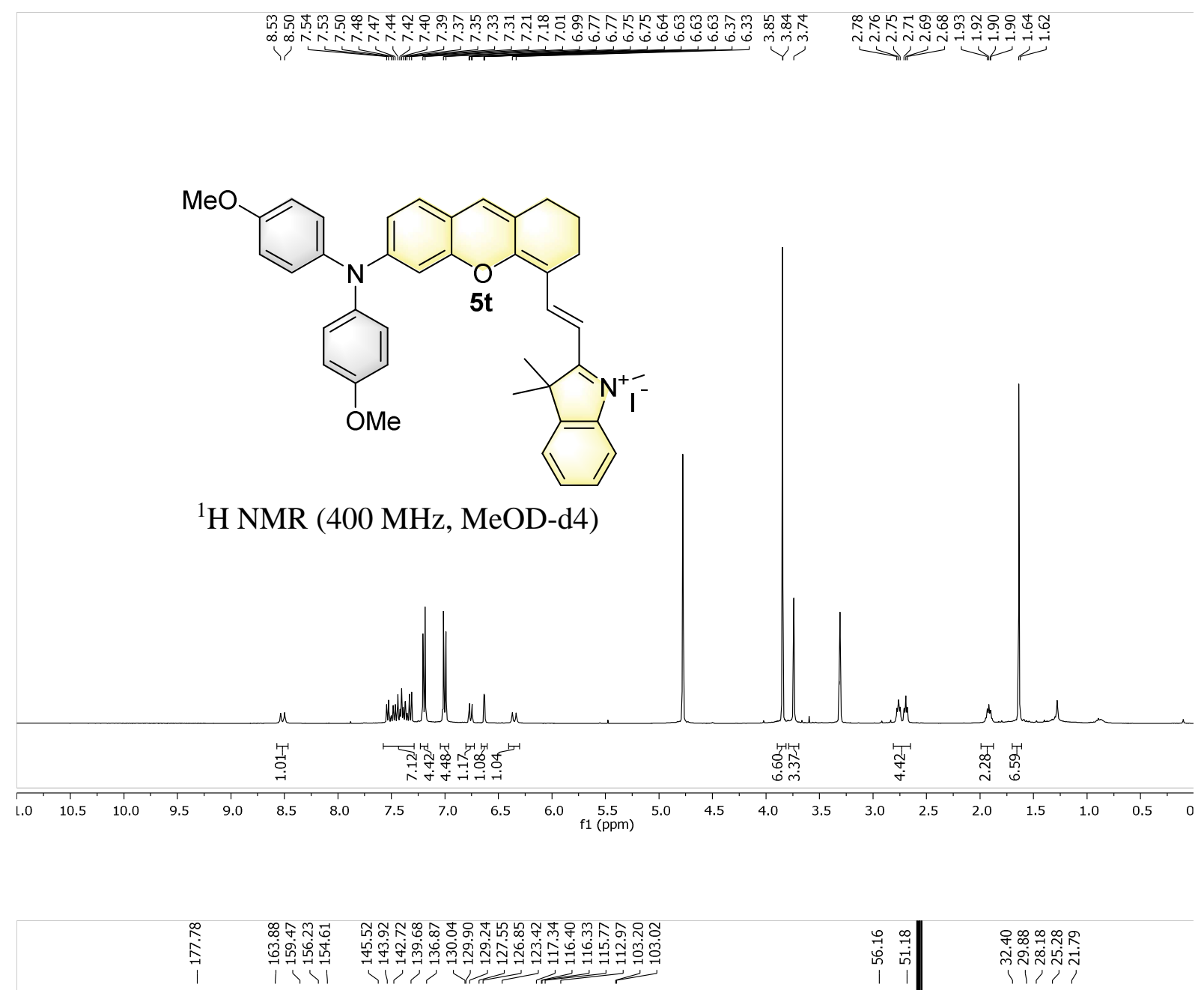

${ }^{13} \mathrm{C}$ NMR (100 MHz MeOD-d4)

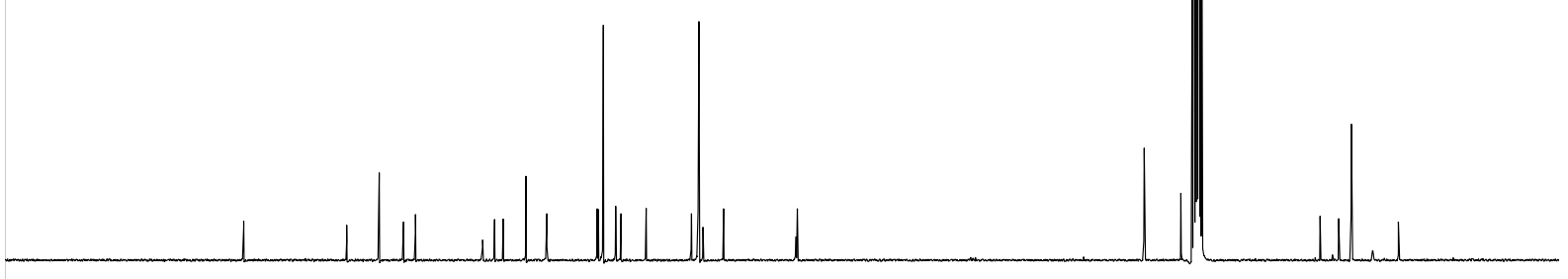

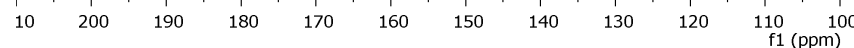




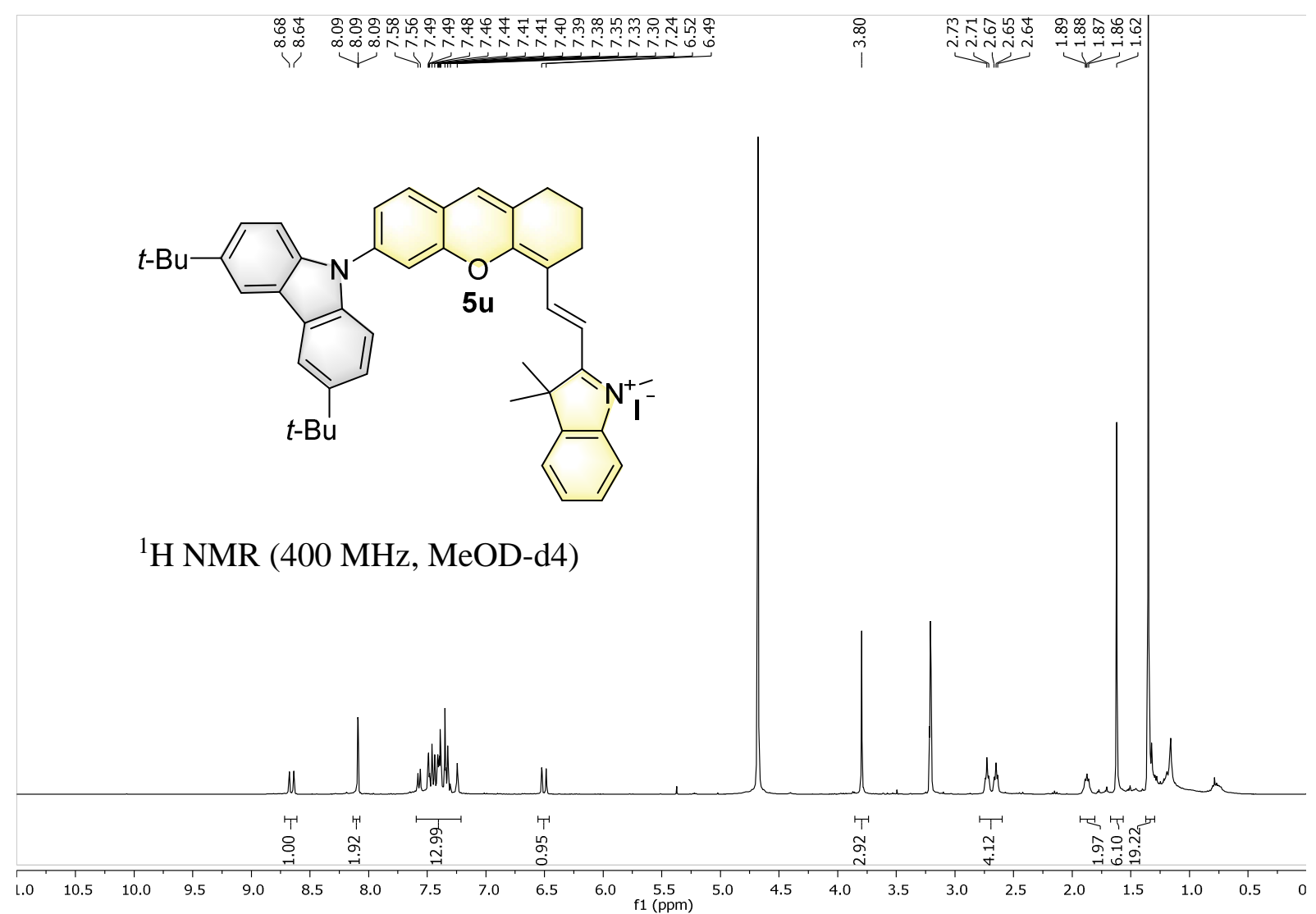

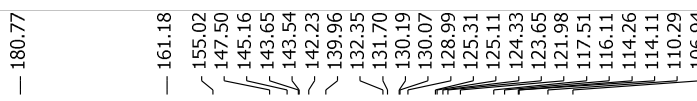

${ }^{13} \mathrm{C}$ NMR (100 MHz MeOD-d4)

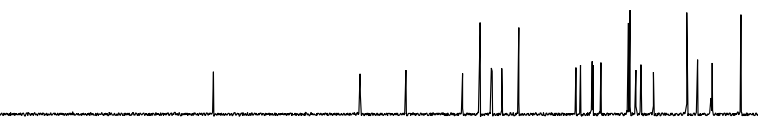

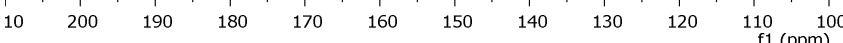




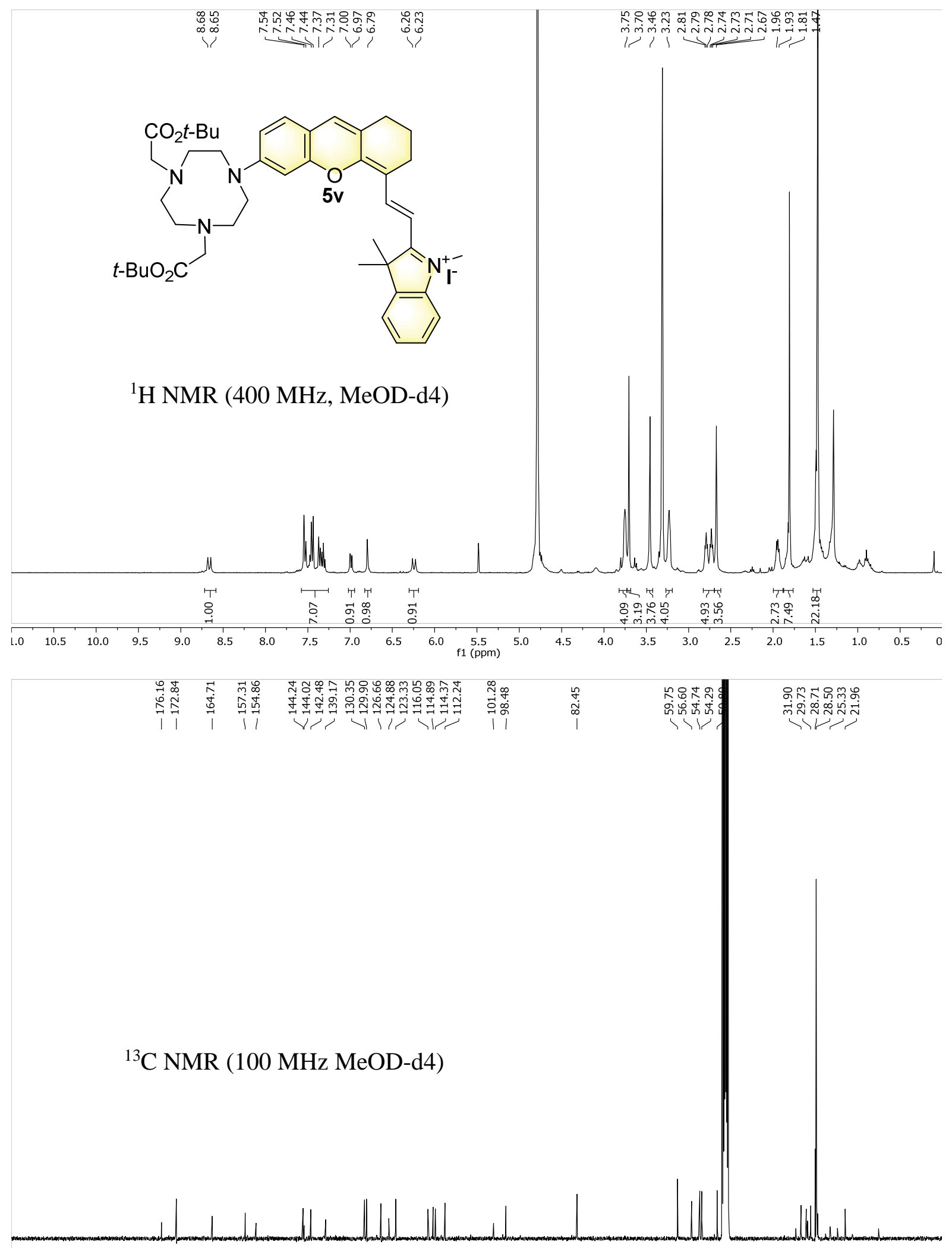

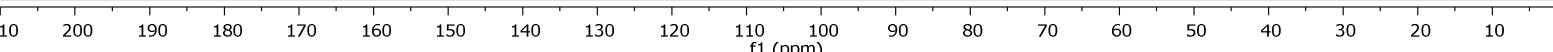




\section{Absorption, excitation and emission spectra of DHX-hemicyanine fused NIR dyes}

Please note: for all absorption spectra, the main absorption band is assigned to $S O \rightarrow S 1$ transition. The second one (far less intense) located in the range 450-500 $\mathrm{nm}$ is assigned to $S O \rightarrow S 2$ transition. For some excitation spectra, peak at $415 \mathrm{~nm}\left(\lambda_{\text {ex }} / 2\right)$ assigned to Rayleigh scattering is observed. All emission spectra (also those of standard namely ICG) are corrected until $850 \mathrm{~nm}$, which explains the artefact observed at this wavelength. UV-vis spectra recorded in the range $10^{-6}-10^{-5} \mathrm{M}$ for determination of molar extinction coefficients. Fluorescence Ex/Em spectra recorded in the range $10^{-7}-10^{-6} \mathrm{M}$ for $Q Y$ determinations.

Fig S1. Normalised absorption (blue), excitation (Em. $830 \mathrm{~nm}$, green) and emission (Ex. 650 $\mathrm{nm}$, red) spectra of compound $\mathbf{5 c}$ in PBS $+5 \%$ BSA at $25^{\circ} \mathrm{C}$.

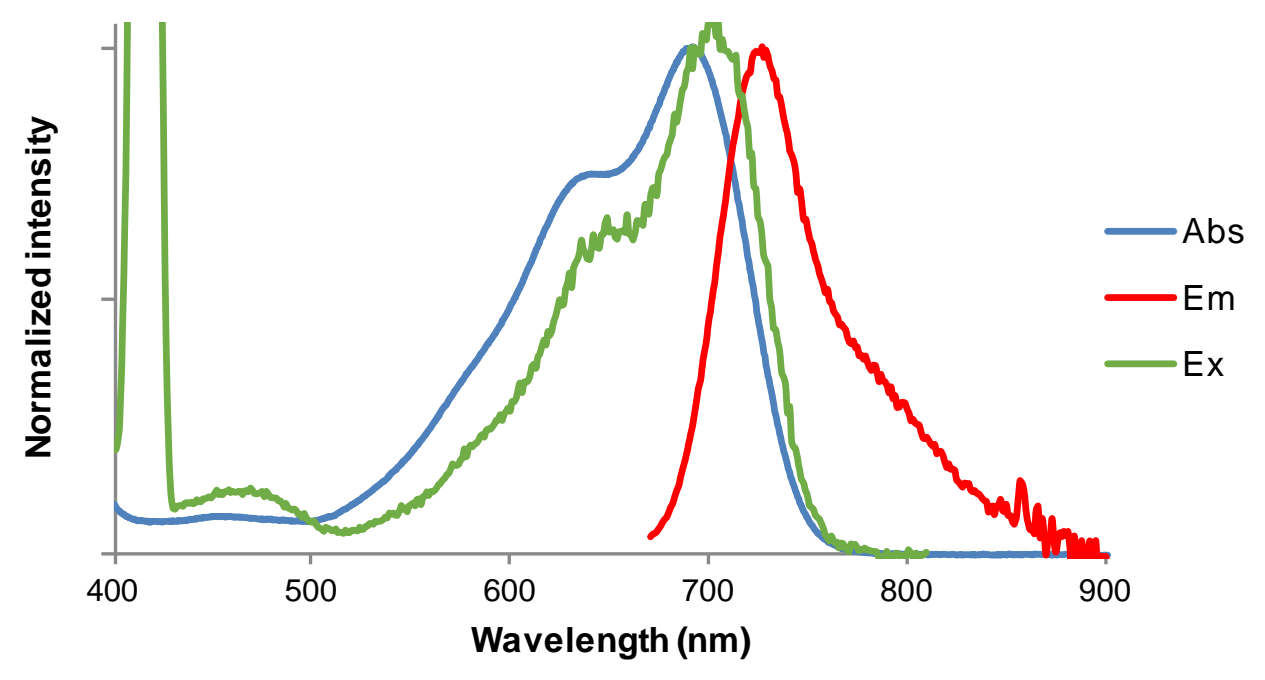

For this compound in PBS + 5\% BSA, no perfect matching between the absorption and excitation spectra was obtained, indicating the presence of H-type dimers in the solution despite the presence of a large amount of BSA.

Fig S2. Normalised absorption (blue), excitation (Em. $830 \mathrm{~nm}$, green) and emission (Ex. 650 $\mathrm{nm}$, red) spectra of compound $\mathbf{5 c}$ in EtOH at $25^{\circ} \mathrm{C}$.

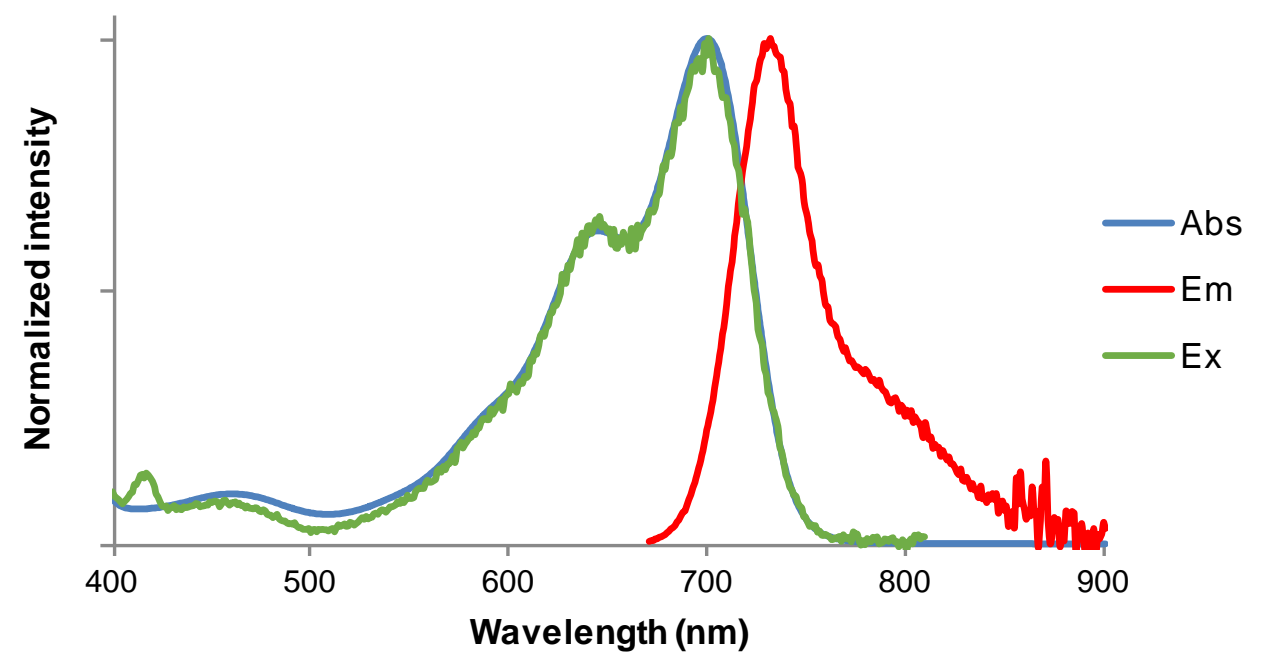


Fig S3. Normalised absorption (blue), excitation (Em. $830 \mathrm{~nm}$, green) and emission (Ex. 650 nm, red) spectra of compound $\mathbf{5 c}$ in $\mathrm{CHCl}_{3}$ at $25^{\circ} \mathrm{C}$.

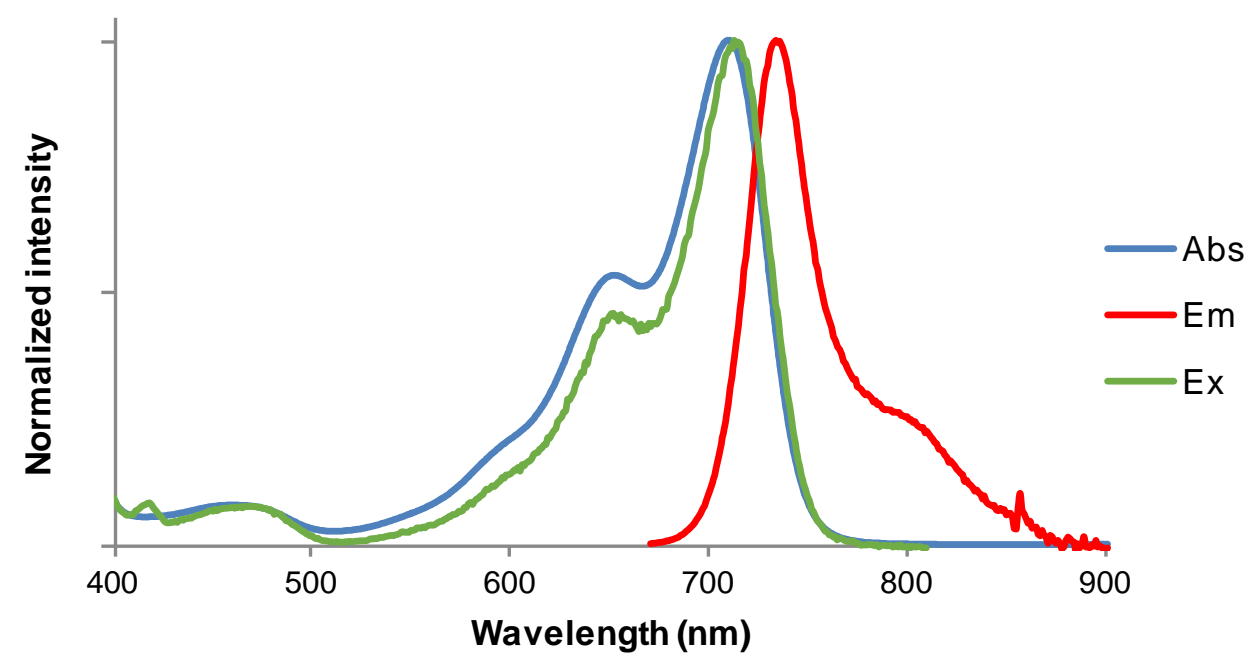

Fig S4. Normalised absorption (blue), excitation (Em. $830 \mathrm{~nm}$, green) and emission (Ex. 650 $\mathrm{nm}$, red) spectra of compound $\mathbf{5 j}$ in PBS $+5 \%$ BSA at $25^{\circ} \mathrm{C}$.

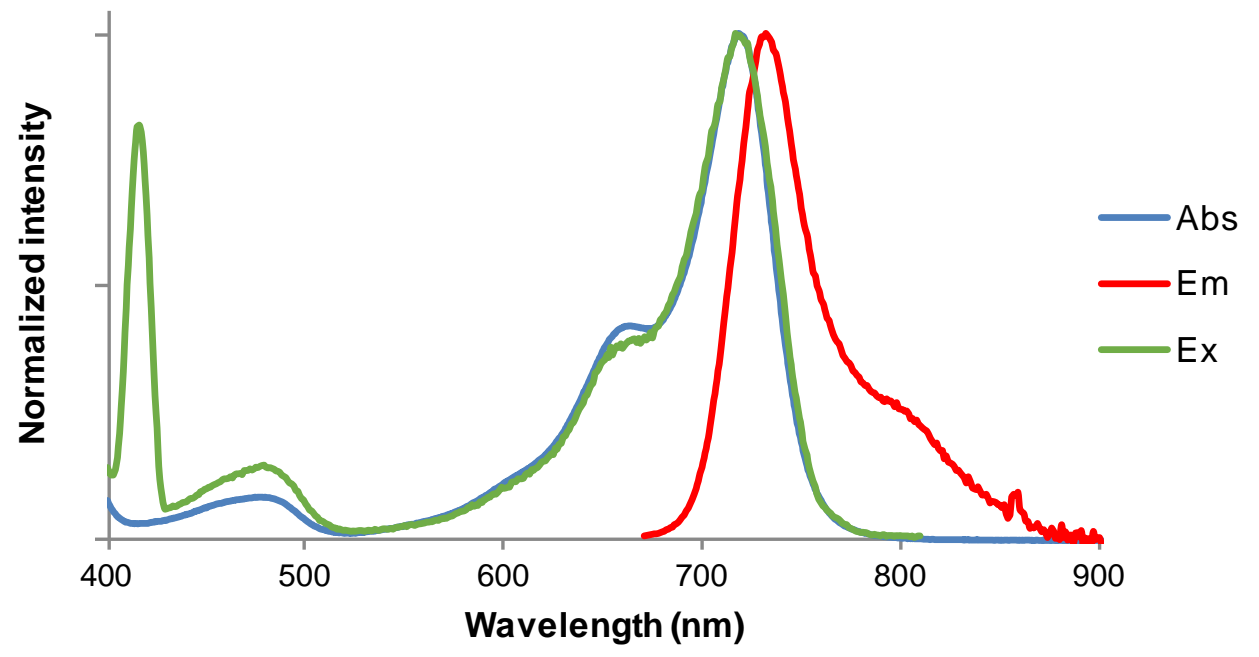


Fig S5. Normalised absorption (blue), excitation (Em. $830 \mathrm{~nm}$, green) and emission (Ex. 650 $\mathrm{nm}$, red) spectra of compound $\mathbf{5} \mathbf{j}$ in $\mathrm{EtOH}$ at $25^{\circ} \mathrm{C}$.

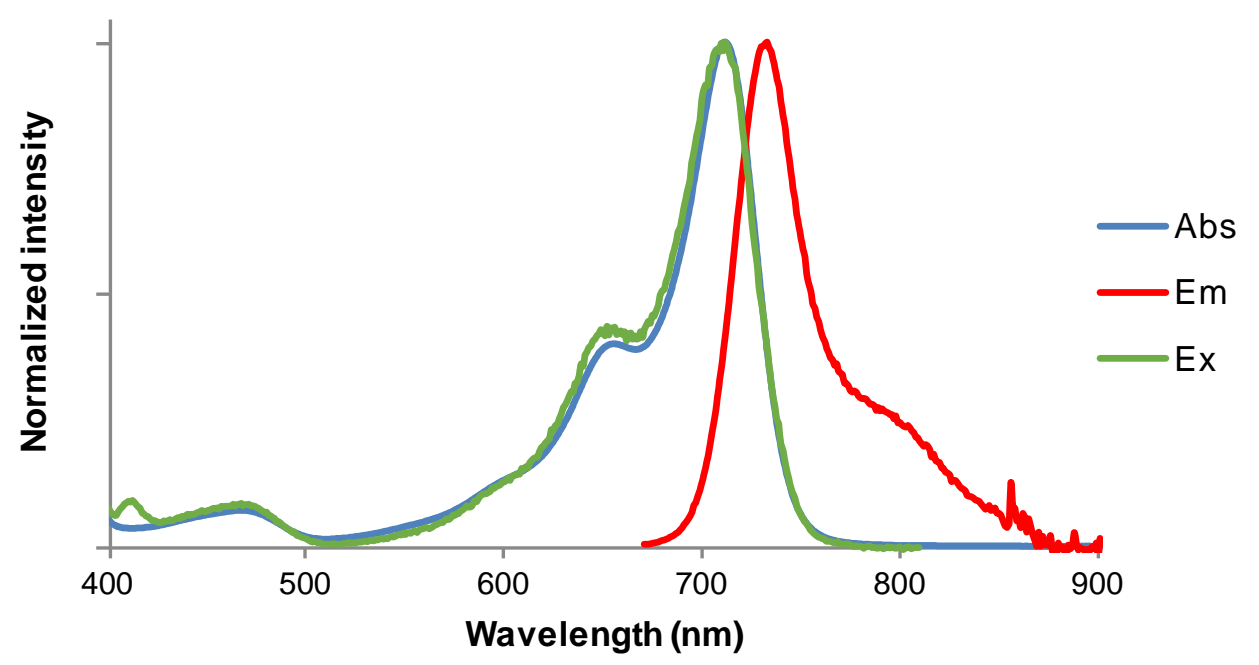

Fig S6. Normalised absorption (blue), excitation (Em. $830 \mathrm{~nm}$, green) and emission (Ex. 650 $\mathrm{nm}$, red) spectra of compound $\mathbf{5 j}$ in $\mathrm{CHCl}_{3}$ at $25{ }^{\circ} \mathrm{C}$.

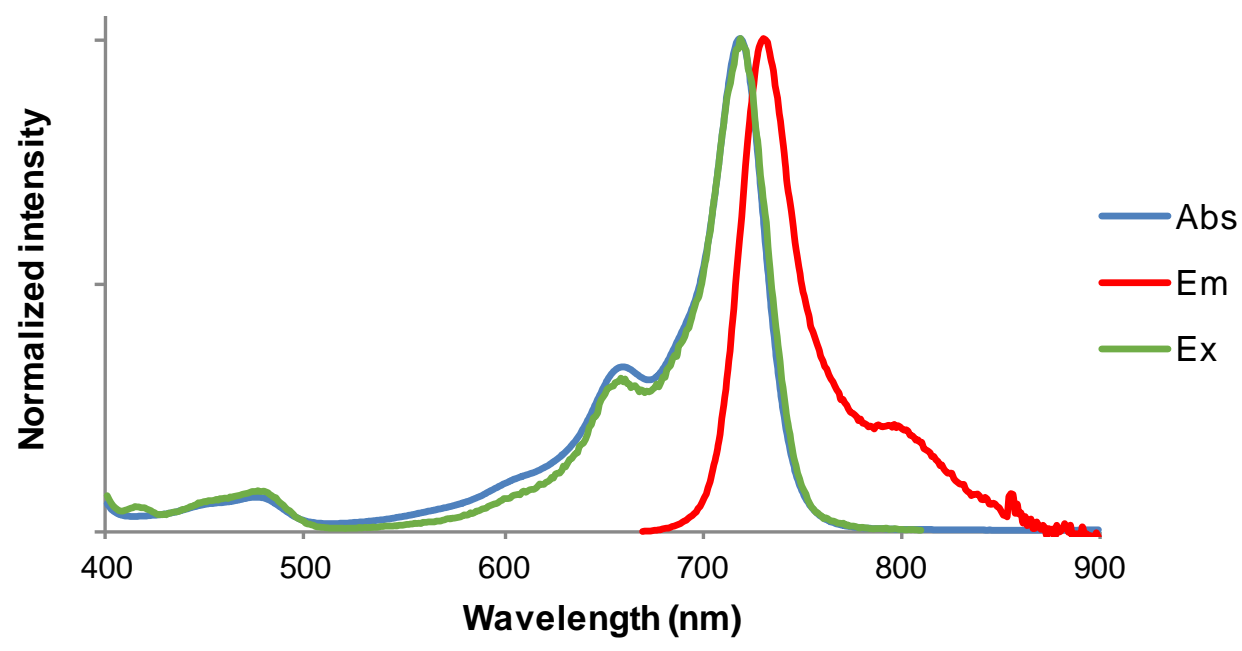


Fig S7. Normalised absorption (blue), excitation (Em. $800 \mathrm{~nm}$, green) and emission (Ex. 650 $\mathrm{nm}$, red) spectra of compound $\mathbf{5 l}$ in PBS $+5 \%$ BSA at $25^{\circ} \mathrm{C}$.

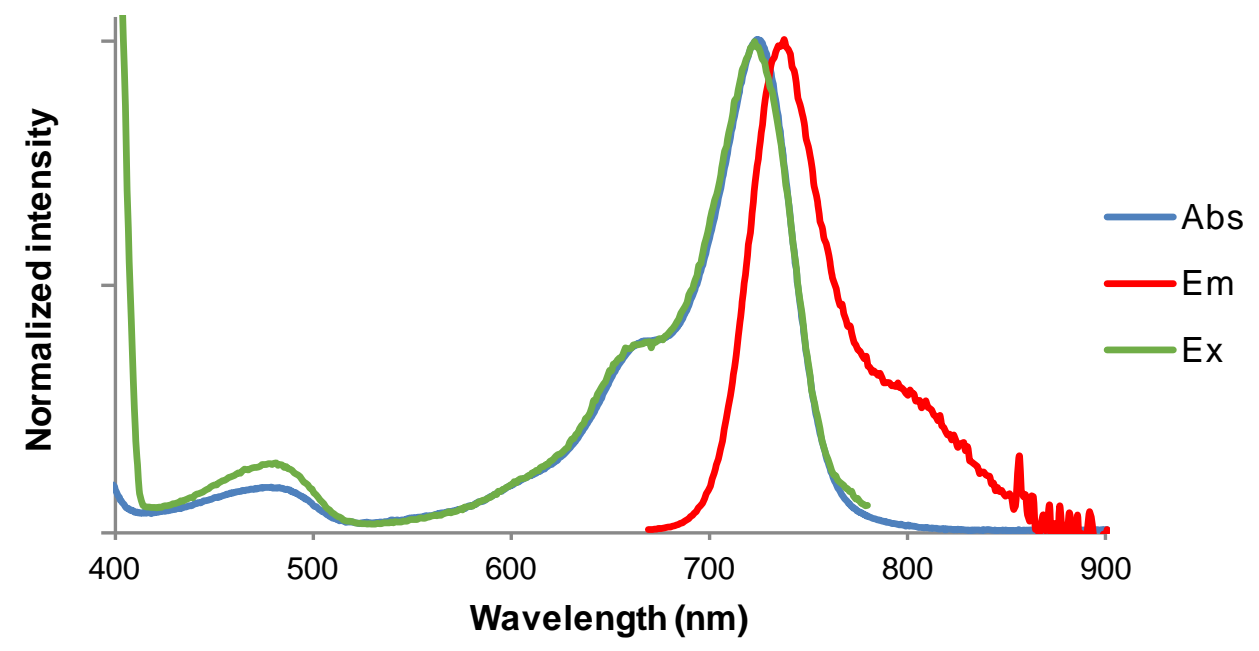

Fig S8. Normalised absorption (blue), excitation (Em. $800 \mathrm{~nm}$, green) and emission (Ex. 650 $\mathrm{nm}$, red) spectra of compound $\mathbf{5 l}$ in EtOH at $25^{\circ} \mathrm{C}$.

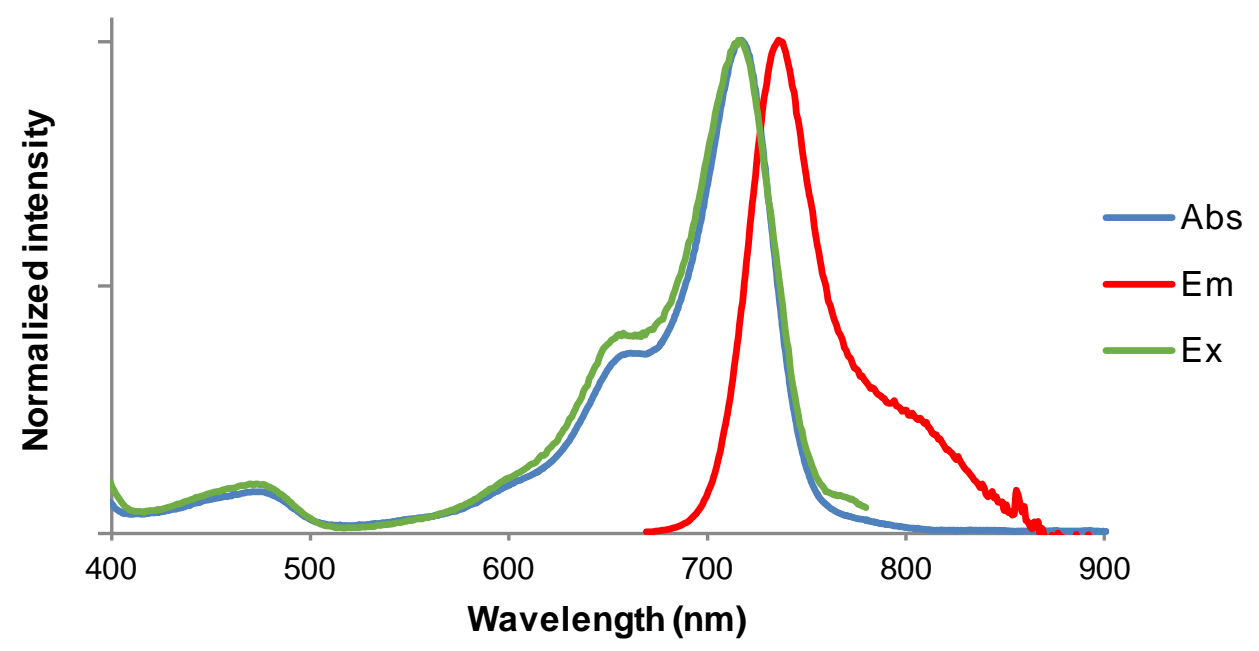


Fig S9. Normalised absorption (blue), excitation (Em. $800 \mathrm{~nm}$, green) and emission (Ex. 650 $\mathrm{nm}$, red) spectra of compound $\mathbf{5 l}$ in $\mathrm{CHCl}_{3}$ at $25^{\circ} \mathrm{C}$.

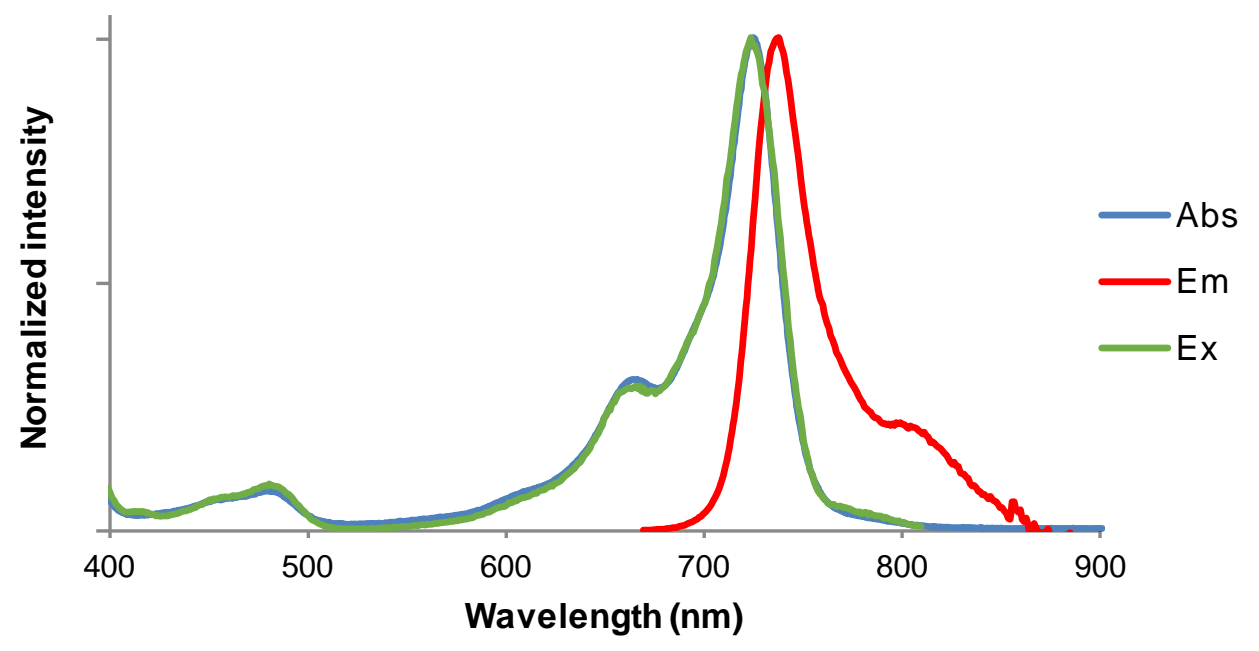

Fig S10. Normalised absorption (blue), excitation (Em. $830 \mathrm{~nm}$, green) and emission (Ex. 650 $\mathrm{nm}$, red) spectra of compound $\mathbf{5 p}$ in PBS $+5 \%$ BSA at $25^{\circ} \mathrm{C}$.

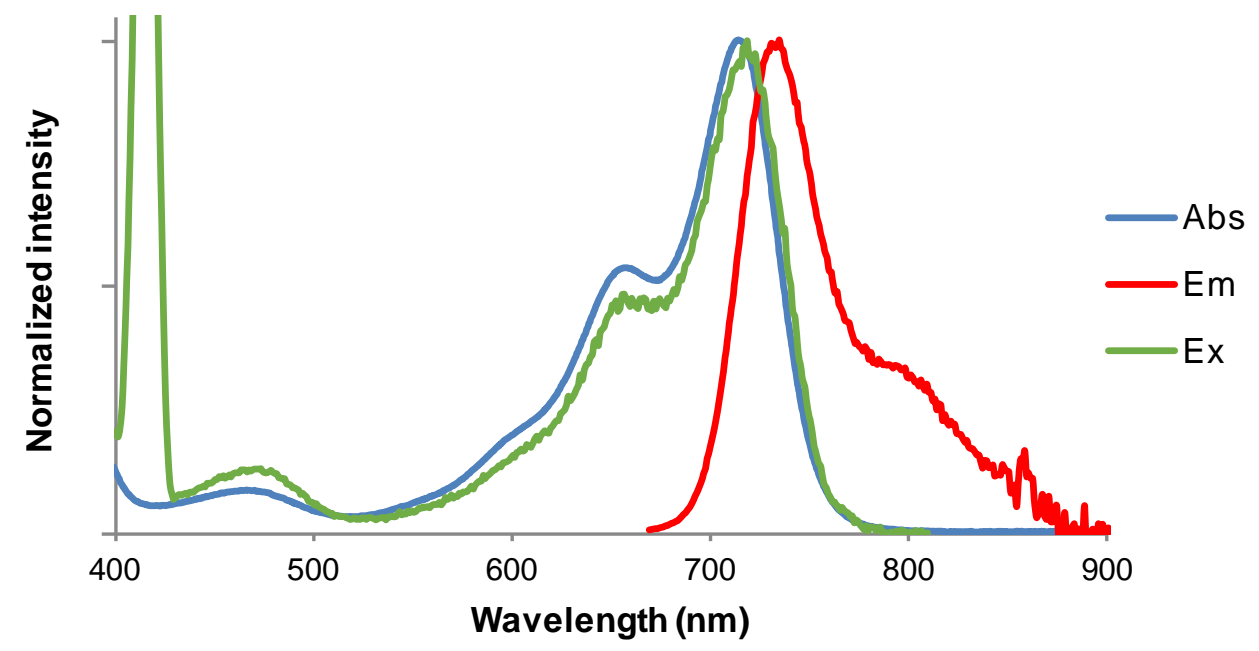


Fig S11. Normalised absorption (blue), excitation (Em. $830 \mathrm{~nm}$, green) and emission (Ex. 650 $\mathrm{nm}$, red) spectra of compound $\mathbf{5 p}$ in EtOH at $25^{\circ} \mathrm{C}$.

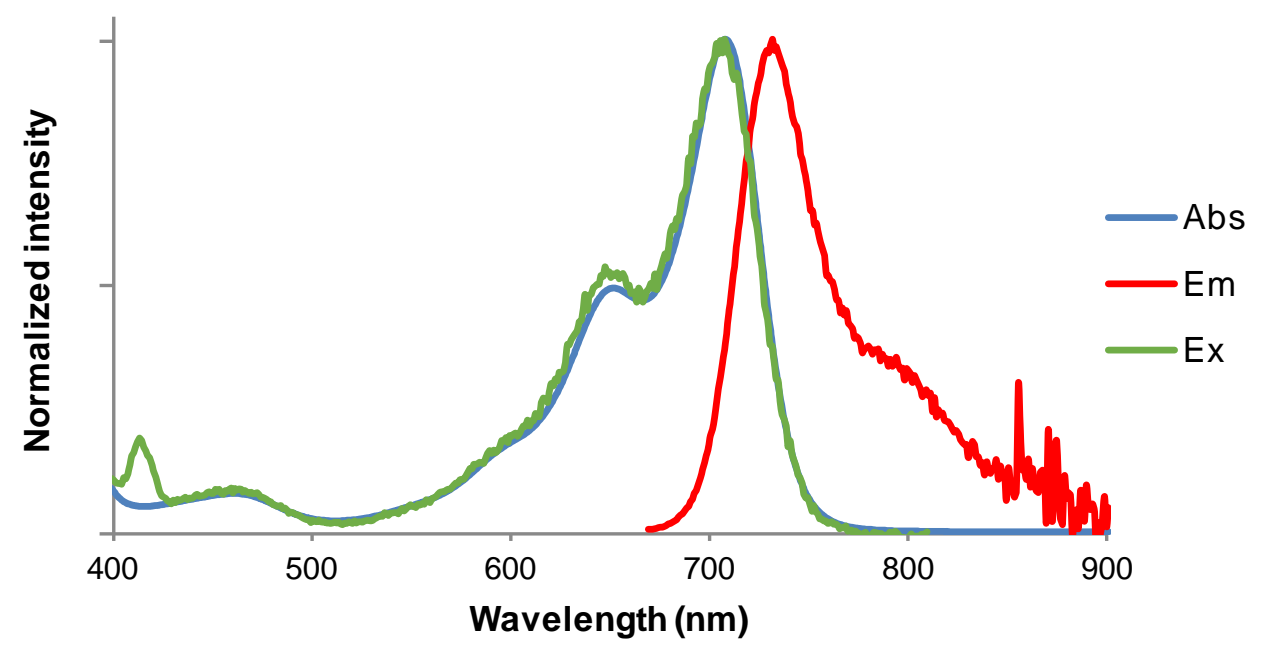

Fig S12. Normalised absorption (blue), excitation (Em. $830 \mathrm{~nm}$, green) and emission (Ex. 650 $\mathrm{nm}$, red) spectra of compound $\mathbf{5 p}$ in $\mathrm{CHCl}_{3}$ at $25^{\circ} \mathrm{C}$.

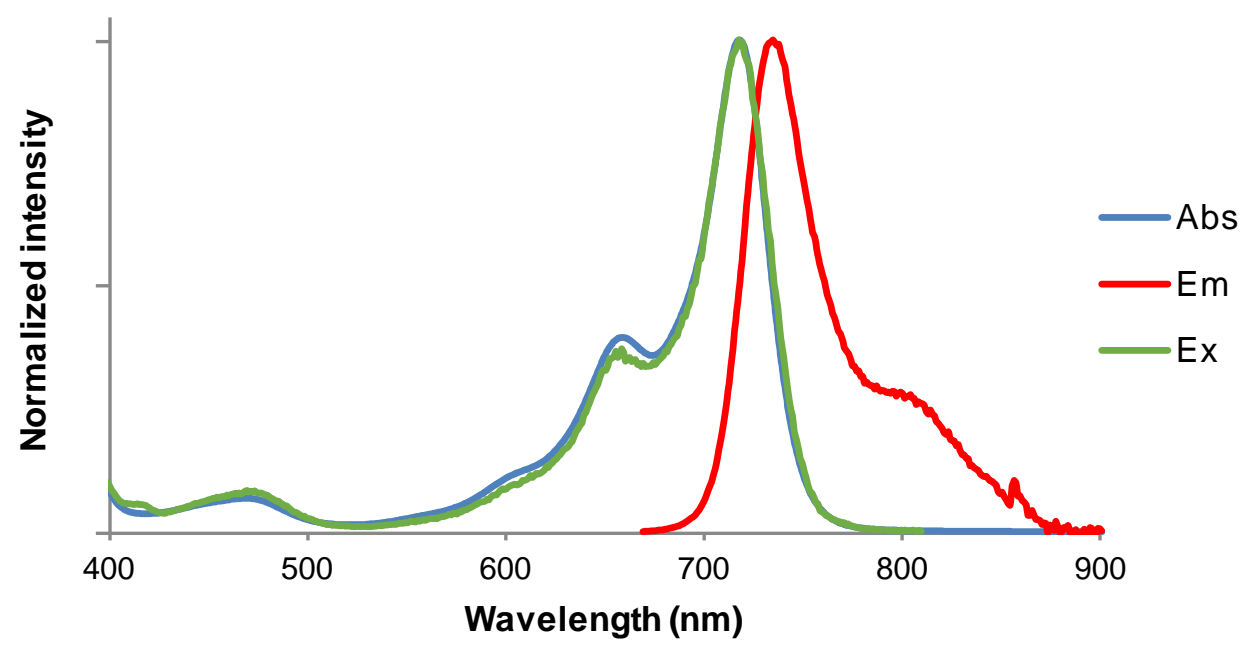


Fig S13. Normalised absorption (blue), excitation (Em. $830 \mathrm{~nm}$, green) and emission (Ex. 650 $\mathrm{nm}$, red) spectra of compound $\mathbf{5 q}$ in $\mathrm{PBS}+5 \%$ BSA at $25^{\circ} \mathrm{C}$.

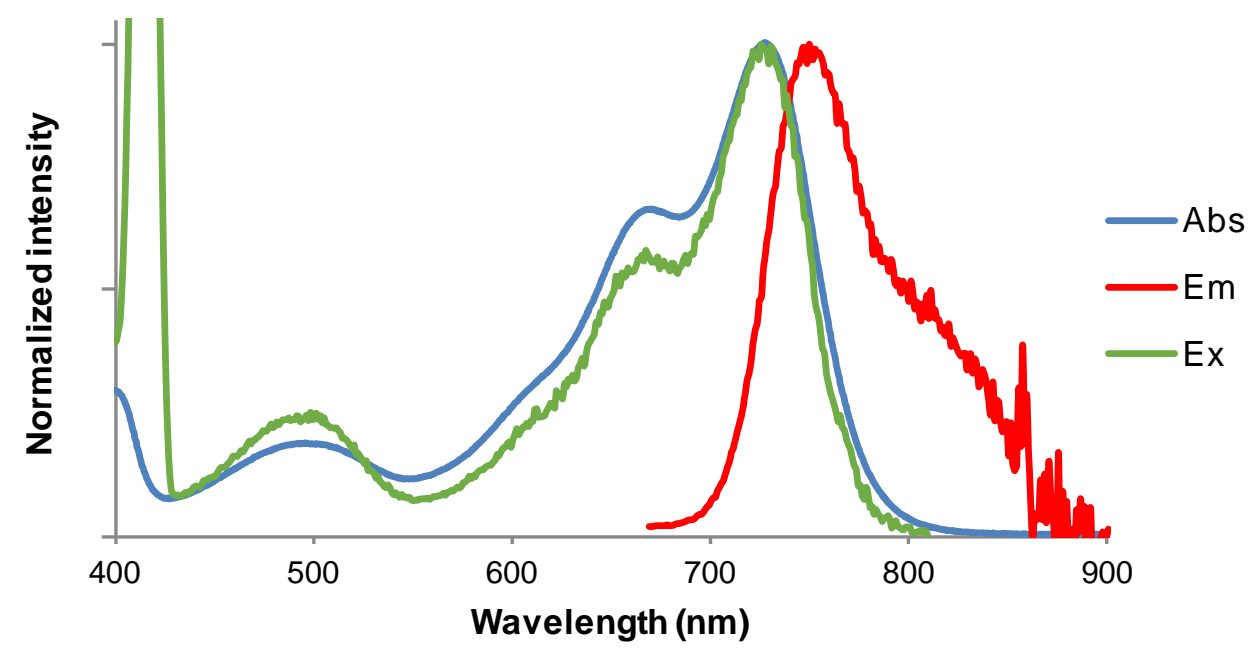

Fig S14. Normalised absorption (blue) and emission (Ex. $650 \mathrm{~nm}$, red) spectra of compound $\mathbf{5 q}$ in $\mathrm{EtOH}$ at $25^{\circ} \mathrm{C}$ (this compound is not fluorescent in EtOH).

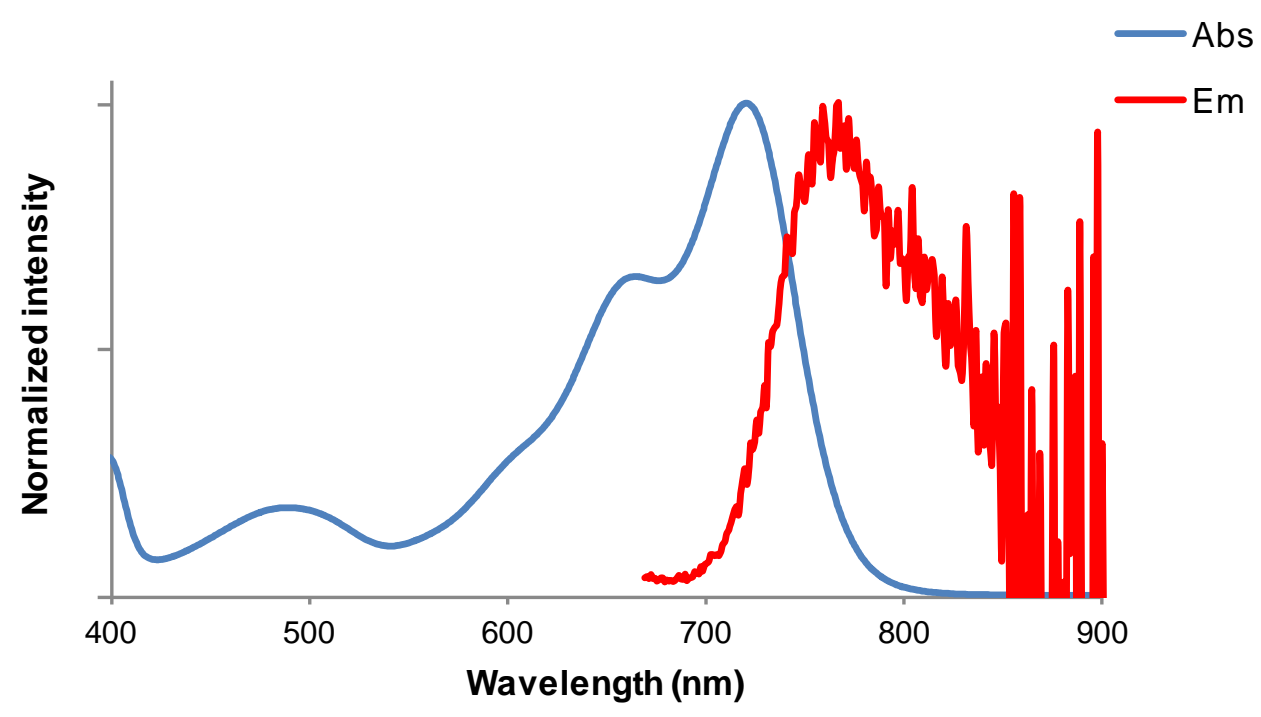


Fig S15. Normalised absorption (blue), excitation (Em. $830 \mathrm{~nm}$, green) and emission (Ex. 650 $\mathrm{nm}$, red) spectra of compound $\mathbf{5 q}$ in $\mathrm{CHCl}_{3}$ at $25^{\circ} \mathrm{C}$.

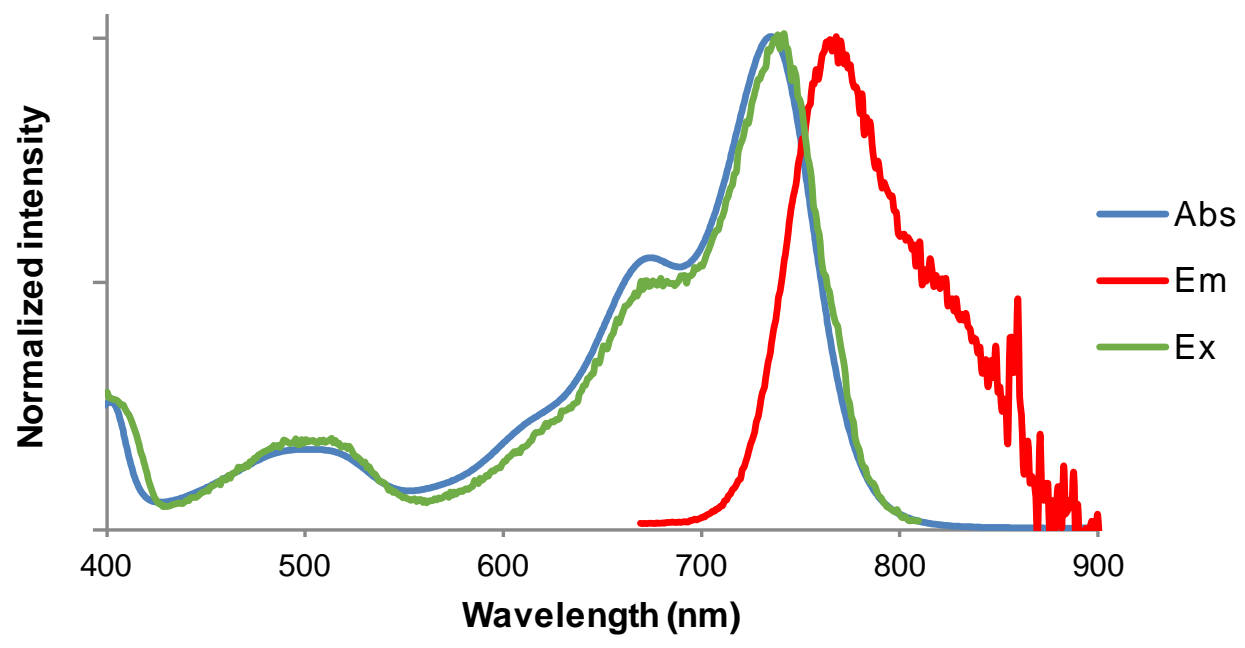

Fig S16. Normalised absorption (blue) and emission (Ex. $650 \mathrm{~nm}$, red) spectra of compound $\mathbf{5 t}$ in $\mathrm{EtOH}$ at $25^{\circ} \mathrm{C}$ (this compound is not fluorescent).

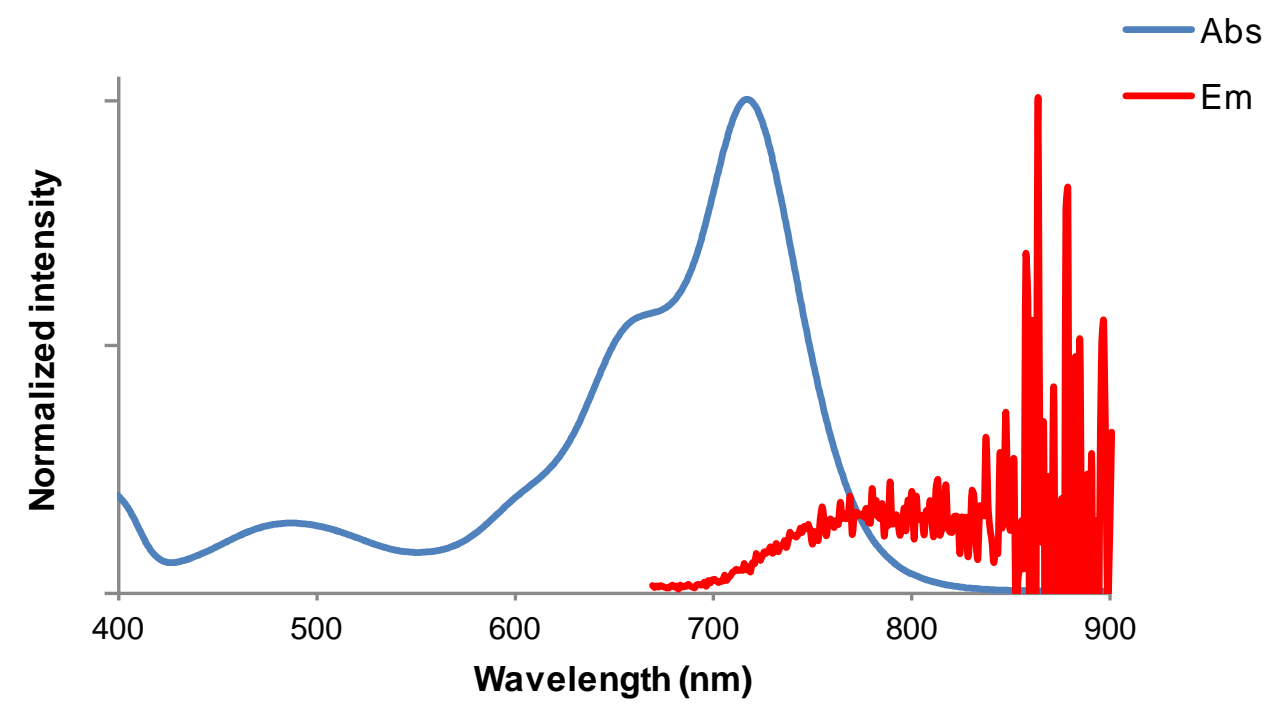


Fig S17. Normalised absorption (blue) and emission (Ex. $650 \mathrm{~nm}$, red) spectra of compound $\mathbf{5 t}$ in $\mathrm{CHCl}_{3}$ at $25^{\circ} \mathrm{C}$ (this compound is not fluorescent).

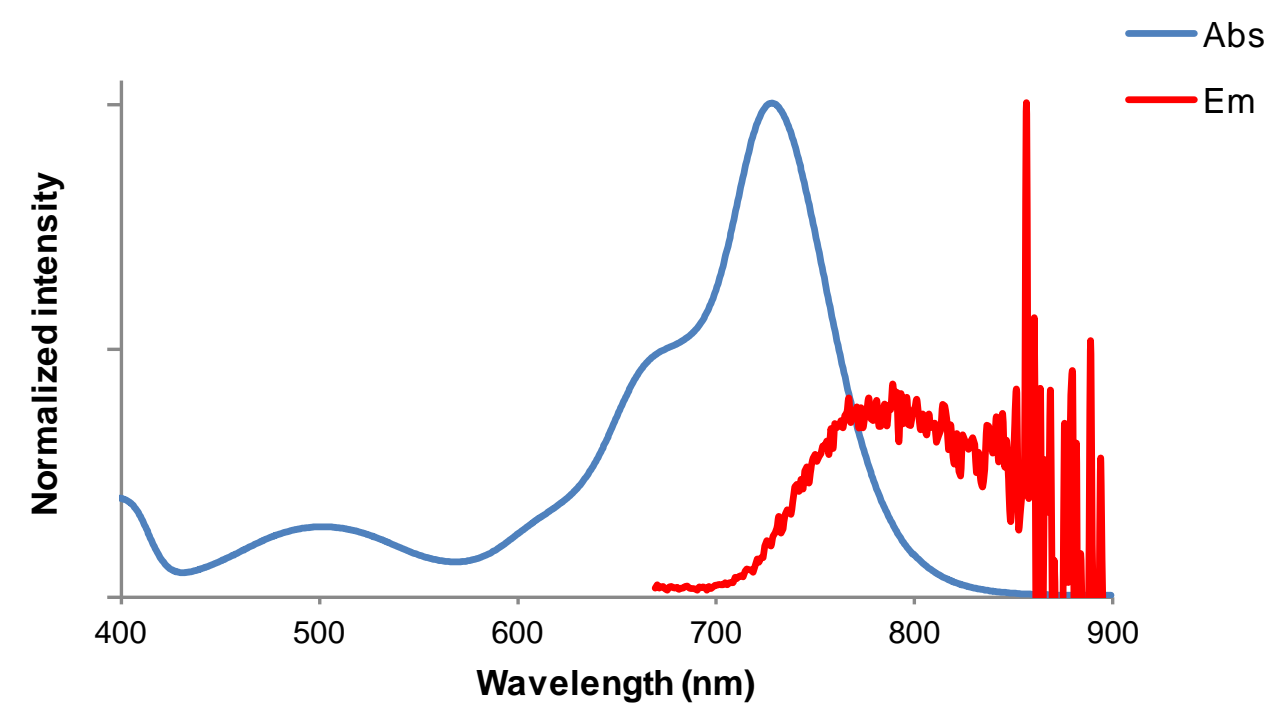

\section{References:}

1. G. R. Fulmer, A. J. M. Miller, N. H. Sherden, H. E. Gottlieb, A. Nudelman, B. M. Stoltz, J. E. Bercaw and K. I. Goldberg, Organometallics, 2010, 29, 2176-2179.

2. A. M. Brouwer, Pure Appl. Chem., 2011, 83, 2213-2228.

3. G. H. Clever, K. Polborn and T. Carell, Angew. Chem. Int. Ed., 2005, 44, 7204-7208.

4. A. Romieu and J.-A. Richard, Tetrahedron Lett., 2016, 57, 317-320. 